\title{
LACUNAS FOR HYPERBOLIC DIFFERENTIAL OPERATORS WITH CONSTANT COEFFICIENTS. II
}

\author{
BY

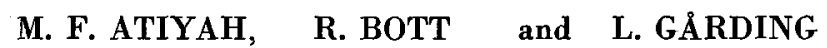
University Harvard University University
of Oxford of Lund

\begin{abstract}
Introduction
Part I of this paper was published in 1970 (see the References [2]). Some of the results on lacunas of hyperbolic operators announced there depend on the fact, proved in all generality by Grothendieck [12] that the cohomology of a non-singular affine algebraic variety can be calculated from its rational differential forms with poles at infinity. Actually, a more precise result was needed putting a bound on the order of the poles. This result is now proved in Chapter I of this paper. It starts with a general account of the whole subject using only the basic results by Serre on algebraic coherent sheaves and Hironaka's resolution of singularities. For curves in the projective plane the results are final (\$6). To obtain the desired bounds on the order of the poles, we use Grothendieck's generalizations of Serre's theorems to the framework of schemes.

In Chapter III we investigate the behaviour of the fundamental solution of a hyperbolic differential operator near the wave front surface. It starts with the observation that the fundamental solution has an analytic continuation across the wave front surface from a given side and at a given point $y$ provided the Petrovsky homology class avoids the intersection of the corresponding hyperplane and the characteristic hypersurface. This condition is called the local Petrovsky condition. The global counterpart, appropriate when $y$ is the origin, is simply that the Petrovsky class vanish. We use the local Petrovsky condition to verify, among other things that the fundamental solution has holomorphic extensions from both sides of a hyperplane part of the wave front surface provided one keeps away from the wave front surface (in the hyperplane) of the corresponding localization of the differential operator. There is also a formula for the jump of the fundamental solution across the hyperplane which shows that the singular support of the fundamental solution does not contain lacunas of the localization provided certain conditions of homogeneity are satisfied. This result connects the local Petrovsky condi10-732907 Acta mathematica 131. Imprimé le 11 Décembre 1973
\end{abstract}


tion with the global Petrovsky condition in one lower dimension. It is probably one of the keys to a complete investigation of the supports and singular supports of fundamental solutions of homogeneous hyperbolic differential operators.

The last paragraph of the paper, entitled Local hyperbolicity, is there to take care of certain techrical difficulties in the verification of the local Petrovsky condition. It can also be read independently. An expanded version written by L. Gårding [8] has appeared in the Israel Journal of Mathematics.

Apart form this general introduction, all our three Chapters have introductory paragraphs giving motives and main results.

\section{Contents}

Chapter I. Cohomology of algebraic varieties . . . . . . . . . . . . . . . . . 146

1. Introduction . . . . . . . . . . . . . . . . . . . . 146

2. Sheaf cohomology . .. . . . . . . . . . . . . . . . 148

3. Algebraic geometry . . . . . . . . . . . . . . . . . . 152

4. The algebraic de Rham theorem . . . . . . . . . . . . . . . . 156

5. Complements of hypersurfaces . . . . . . . . . . . . . . . . . 161

6. Plane curves . . . . . . . . . . . . . . . . . . . . . . 167

Chapter II. Applications to the theory of lacunas . . . . . . . . . . . . . . . . 171

7. Introduction . . . . . . . . . . . . . . . . . . . . 171

8. Homology of hyperbolic hypersurfaces . . . . . . . . . . . . . . 176

9. Lacunas. Proofs of the main results . . . . . . . . . . . . . . . 177

Chapter III. Sharp fronts. The local Petrovsky condition . . . . . . . . . . . . . 180

10. Introduction . . . . . . . . . . . . . . . . . . . . . 180

11. Representatives of the Petrovsky class . . . . . . . . . . . . . . 183

12. Sharpness at regular quadratic points . . . . . . . . . . . . . . 186

13. Weak sharpness across plane parts of the wave front surface . . . . . . . . 190

14. Holomorphic sharpness across plane parts of the wave front surface . . . . . . 194

15. Local hyperbolicity . . . . . . . . . . . . . . . . . . . . . 199

\section{CHAPTER I. HOMOLOGY OF ALGEBRAIC VARIETIES}

\section{\$ 1. Introduction}

Our aim in this chapter is to give an exposition of the "algebraic" de Rham theorem, which asserts that the cohomology of a non-singular affine algebraic variety can be calculated from the complex of algebraic differential forms (i.e. holomorphic forms with only poles at $\infty$ ). Since an affine variety is a special kind of Stein manifold, this theorem 
can be regarded as a strengthening of the theorem that the cohomology of a Stein manifold is given by the complex of all holomorphic differential forms (with no restrictions at $\infty)$. As is well known the theorem for Stein manifolds is an easy consequence of the holomorphic Poincaré Lemma and the vanishing theorem for cohomology of coherent sheaves (the famous Theorem B of Cartan). In the algebraic case the proof depends similarly on a kind of Poincaré Lemma at $\infty$ and on the Kodaira vanishing theorem for projective varieties: the essential point is that we must work with some projective compactification $\bar{X}$ of our affine variety $X$. In the simplest case $X \subset \mathbf{C}^{N}, \bar{X} \subset P_{N}(\mathbf{C})$ and $\bar{X}$ and $Y=\bar{X}-X$ are both assumed non-singular. Because $Y$ (the part at $\infty$ ) is nonsingular the "Poincaré Lemma at $\infty$ " is easily dealt with. Because $Y$ is a hyperplane section of $\bar{X}$ the Kodaira vanishing theorem applies and the de Rham theorem then follows. This proof is due to Atiyah-Hodge [3], as is the slight generalization in which $Y$ is a union of non-singular components which meet transversally (normal crossings). In general it is not possible to embed $X$ in $\mathbf{C}^{N}$ so that $\bar{X}, Y$ have the above simple properties. However, Hironaka's basic theorem on the resolution of singularities asserts that we can find a non-singular compactification $\bar{X}$ of $X$ so that $Y=\bar{X}-X$ has only normal crossings. We must however drop the condition that $Y$ is a hyperplane section of $\bar{X}$ (i.e. the compactification is not induced by $\mathbf{C}^{N} \subset P_{N}$ ): in fact $\bar{X}$ is obtained by taking any compactification $\tilde{X}$ (induced say by $\mathbf{C}^{N} \subset P_{N}$ ) and then blowing up the singularities of $\tilde{X}$ at $\infty$. Locally at $\infty$ we are in as good a situation as before but the Kodaira vanishing theorem no longer applies. However Grothendieck [12] observed that there is a slightly weaker vanishing theorem that can be proved in this case and is sufficient to prove the general de Rham theorem.

Since Grothendieck's treatment is rather concise we shall give here a complete proof of the algebraic de Rham theorem (on the lines indicated above) using only the basic results of Serre [17], [19] on algebraic coherent sheaves, and of course Hironaka's resolution of singularities.

As explained in Part I, we also need some refinements and extensions of the de Rham theorem. In one direction we need to consider algebraic families $X_{t}(t \in T$, algebraic) and to prove that the cohomology of $X_{t}$ can be obtained from forms with poles of order $k$ at $\infty$ where $k$ is independent of $t$. In application $X_{t}=P_{n}-Y_{t}$ where $\left\{Y_{t}\right\}$ is the family of all hypersurfaces of degree $m$. To get this bound we need to put a parameter into our proof and we then need the analogoues of some of Serre's theorems "with parameters". Such questions fall naturally into the framework of Grothendieck's "schemes" and we shall need therefore to use Grothendieck's generalizations of Serre's theorems (notably the finite-dimensionality of cohomology on projective varieties). 
In the preceding theorems the bound on the order of pole required depends on

(a) the resolution process and

(b) the vanishing theorem.

In the non-singular case when (a) is not required it therefore depends only on (b). If moreover we are dealing (as in our applications) with hypersurfaces in projective space, then the vanishing theorems are explicit enough to give explicit bounds for the orders of pole. In these cases therefore we get more explicit results and these in turn give more refined results on lacunas.

In low dimensions (essentially curves in the plane) it is possible to obtain the refined results without assuming non-singularity. This leads to correspondingly strong statements for lacunas in low dimensions. For the convenience of the reader we shall collect in the next section the various technical results on sheaf cohomology that will be required (mainly the use of spectral sequences) and in $\S 3$ the basic results from algebraic geometry that will be required (Serre, Grothendieck, Hironaka). The remaining sections will then be devoted to deducing the algebraic de Rham theorem and its various refinements.

\section{§ 2. Sheaf Cohomology}

A general reference for this section is Godement [9].

We recall that a sheaf $S$ on a space $X$ has cohomology groups $H^{q}(X, S) q \geqslant 0$, that $H^{0}(X, S)=\Gamma(X, S)$ is the space of sections and that an exact sequence of sheaves

$$
0 \rightarrow S^{\prime} \rightarrow S \rightarrow S^{\prime \prime} \rightarrow 0
$$

gives rise to a long exact cohomology sequence

$$
0 \rightarrow H^{0}\left(X, S^{\prime}\right) \rightarrow \ldots H^{q}\left(X, S^{\prime}\right) \rightarrow H^{q}(X, S) \rightarrow H^{q}\left(X, S^{\prime \prime}\right) \stackrel{\delta}{\rightarrow} H^{q+1}\left(X, S^{\prime}\right) \rightarrow \ldots
$$

The $H^{q}$ are defined in general using a resolution of $S$ by injective or flabby sheaves. However in certain situations, notably when $X$ is paracompact Hausdorff, the $H^{q}$ can also be defined by the Cech method using open coverings. We recall that if $\mathcal{U}=\left\{\mathcal{U}_{i}\right\}$ is an open covering we can form a cochain complex $C^{*}(X, \mathcal{U})$ where $C^{q}(X, \mathcal{U})$ consists of alternating functions

$$
\left(i_{0}, \ldots, i_{q}\right) \mapsto f\left(i_{0}, \ldots, i_{q}\right) \in \Gamma\left(u_{i 0} \cap \ldots \mathcal{U}_{i q}, S\right),
$$

and that the Cech groups $\breve{H}^{q}$ are defined by

$$
\check{H}^{\alpha}(X, S)=\lim _{\vec{u}} H^{a}\left(C^{*}(\mathcal{U}, S)\right) .
$$

Moreover if for every subset $\left(i_{0}, \ldots, i_{q}\right)$ (and all $q$ ) we have 


$$
H^{p}\left(\mathcal{U}_{t_{0}} \cap \ldots \mathcal{U}_{i_{g}}, S\right)=0 \text { for } p \geqslant 1
$$

then the limit is unnecessary and

$$
H^{q}(X, S)=H^{q}\left(C^{*}(\mathcal{U}, S)\right) .
$$

The de Rham theorem on a manifold is concerned with the complex $\Omega^{*}$ of differential forms:

$$
0 \rightarrow \Omega^{0} \stackrel{d}{\rightarrow} \Omega^{1} \stackrel{d}{\rightarrow} \Omega^{2} \rightarrow \ldots \rightarrow \Omega^{n} \rightarrow 0
$$

and the proofs of the various de Rham theorems are simplified by using the notion of hypercohomology of a complex of sheaves. This is a generalization of the cohomology of a single sheaf, and reduces to it when the complex has only one non-zero term. The general definition of hypercohomology is due to Cartan-Eilenberg [7] and involves using a special kind of resolution for the complex. As with ordinary cohomology there is a Cech definition which works on paracompact Hausdorff spaces and is easier to define. For any open covering $\mathcal{U}$ of $X$ and any complex $\Omega^{*}$ of sheaves the cochains $C^{*}\left(\mathcal{U}, \Omega^{*}\right)$ form a double complex, one differential coming from the covering and the other from $\Omega^{*}$. The Cech hypercohomology groups $\mathcal{H}^{q}$ may then be defined by

$$
\mathcal{H}^{q}\left(X, \Omega^{*}\right)=\lim _{\vec{u}} H^{q}\left(C^{*}\left(\mathcal{U}, \Omega^{*}\right)\right)
$$

where $H^{q}\left(C^{*}\left(\mathcal{U}, \Omega^{*}\right)\right)$ denotes the cohomology of the single complex associated in the usual way to the double complex. Again if (2.1) is satisfied with $S=\Omega^{q}$ (for all $q$ ) the limit is unnecessary.

As is well known(1) the cohomology of a double complex (with total differential $d=d^{\prime}+d^{\prime \prime}$ ) can be computed in steps, either starting with $d^{\prime}$ or else starting with $d^{\prime \prime}$. Applied here this gives rise to two spectral sequences converging to the hypercohomology. $\left({ }^{2}\right)$ In one spectral sequence (using the differential on $\Omega^{*}$ first) we have

$$
E_{2}^{p, q}=H^{p}\left(X, h^{q}\left(\Omega^{*}\right)\right)
$$

where $h^{q}\left(\Omega^{*}\right)$ is the $q$-th cohomology sheaf of $\Omega^{*}$, i.e. $h^{q}\left(\Omega^{*}\right)$ is the kernel of $\Omega^{q} \rightarrow \Omega^{q+1}$ modulo the image of $\Omega^{q-1} \rightarrow \Omega^{a}$. In the second spectral sequence we have (writing $F$ instead of $E$ to distinguish)

$$
F_{1}^{p, q}=H^{q}\left(X, \Omega^{p}\right)
$$

(1) See Godement [9], p. 86.

( ${ }^{2}$ Each covering $\mathcal{U}$ gives a double complex, hence a spectral sequence, and taking direct limits we obtain a limit spectral sequence. This limit involves no problems since we shall throughout assume that $\operatorname{dim} X<\infty$ and $\Omega *$ is a finite complex. 
and the differential $d_{1}: H^{q}\left(X, \Omega^{p}\right) \rightarrow H^{q}\left(X, \Omega^{p+1}\right)$ is induced by the sheaf homomorphism $\Omega^{p} \rightarrow \Omega^{p+1}$. Thus, in particular,

$$
F_{2}^{p, 0}=D R^{p}\left(\Omega^{*}\right)
$$

is the " $p$ th de Rham group" $D R^{p}$ of the complex $\Omega^{*}$, i.e. the cohomology of the complex $\Gamma\left(X, \Omega^{*}\right)$ of global sections. Note that we always have a natural homomorphism $D R^{p} \rightarrow \mathcal{H}^{p}$ (in spectral sequence terms $\boldsymbol{F}_{2}^{p .0} \rightarrow \boldsymbol{F}_{\infty}^{p, 0}=\mathcal{H}_{p}^{p} / \mathcal{H}_{p+1}^{p}$, but $\mathcal{H}_{p+1}^{p}=0$ ).

With this machinery let us recall how the usual de Rham theorems are proved. We take $\Omega^{*}$ to be the complex of $C^{\infty}$ forms on a $C^{\infty}$ manifold $X$. The Poincaré Lemma asserts that

$$
h^{q}\left(\Omega^{*}\right)=0 \quad \text { for all } q \geqslant 1
$$

and, since the $\Omega^{*}$ are fine sheaves, we have

$$
H^{q}\left(X, \Omega^{p}\right)=0 \quad \text { for } q \geqslant 1 \text { and all } p .
$$

Now (2.5) implies that the spectral sequence $E_{r}$ is trivial $\left(^{(1)}\right.$ and so (since $h^{0}\left(\Omega^{*}\right)=C$, the sheaf of constants)

$$
H^{p}(X, C)=E_{2}^{p, 0} \cong \mathcal{H}^{p}\left(X, \Omega^{*}\right)
$$

Similarly (2.6) implies that the second spectral sequence $\boldsymbol{F}_{r}$ is trivial (for $r \geqslant 2$ ) and so

$$
D R^{p}\left(\Omega^{*}\right)=F_{2}^{p, 0} \cong \mathcal{H}^{p}\left(X, \Omega^{*}\right)
$$

The de Rham theorem then follows by combining (2.7) and (2.8). It can of course be proved without using hypercohomology and spectral sequences: we simply break up $\Omega^{*}$ into short exact sequences, take cohomology groups and use (2.6). However in more complicated situations which we shall encounter the machinery of hypercohomology is very convenient.

On a complex Stein manifold we have a formally similar proof, but this time $\Omega^{*}$ is the complex of holomorphic forms, (2.5) is replaced by the holomorphic Poincaré Lemma and (2.6) is replaced by the much deeper vanishing "Theorem B" of Cartan.

An important property of hypercohomology is the following comparison theorem:

Proposition (2.9). Let $\varphi: \Omega^{*} \rightarrow \tilde{\Omega}^{*}$ be a homomorphism of complexes of sheaves which induces an isomorphism on cohomology sheaves $h^{q}\left(\Omega^{*}\right) \cong h^{q}\left(\tilde{\Omega}^{*}\right)$ for all $q$. Then $\varphi$ induces an isomorphism of hypercohomology $\mathcal{H}^{q}\left(X, \Omega^{*}\right) \cong \mathcal{H}^{q}\left(X, \tilde{\Omega}^{*}\right)$. In particular, if $H^{q}\left(X, \Omega^{p}\right)=$ $H^{q}\left(X, \Omega^{p}\right)=0$ for $q \geqslant 1$ and all $p$, then $\varphi$ induces an isomorphism of de Rham groups $D R^{q}\left(\Omega^{*}\right) \cong D R^{q}\left(\tilde{\Omega}^{*}\right)$.

(1) That is $E_{2}=E_{3}=\ldots=E_{\infty}$. 
Proof. $\varphi$ induces a homomorphism of spectral sequences $E_{r} \rightarrow \widetilde{E}_{r}$. For $r=2$ the assumption on $\varphi$ and (2.2) shows that we have an isomorphism. Hence $E_{r} \cong \widetilde{E}_{r}$ for all $r$ and so the end terms of the spectral sequences, namely the hypercohomology groups, are also isomorphic. If moreover $H^{q}\left(X, \Omega^{p}\right)=0$ for $q \geqslant 1$ then the second spectral sequence $F_{r}^{p, q}$ is trivial for $r \geqslant 2$ and so $\mathcal{H}^{q}\left(X, \Omega^{*}\right) \cong D R^{q}\left(\Omega^{*}\right)$. Similarly for $\tilde{\Omega}^{*}$ and so $\varphi$ induces an isomorphism $D R^{q}\left(\Omega^{*}\right) \rightarrow D R^{q}\left(\tilde{\Omega}^{*}\right)$ as required.

This proposition will be applied in $\$ 4$ to deduce the algebraic de Rham theorem from the $C^{\infty}$ theorem. It will depend on the vanishing theorem (2.6) for the complex $\Omega^{*}$ of meromorphic differentials on $\vec{X}$ with poles on $Y$. In order to get bounds on the orders of pole required we shall need to introduce an increasing sequence of subsheaves

$$
\Omega^{*}(k) \subset \Omega^{*}(k+1) \subset \ldots
$$

with $\Omega^{*}=\lim \Omega^{*}(k)$, where $k$ denotes (roughly speaking) the order of pole. We shall then need the following rather technical lemma which puts bounds into the spectral sequence of $(2.9)$.

LEMMA (2.10). Let $\Omega^{*}(k) \subset \Omega^{*}(k+1) \subset \ldots$ be an increasing sequence of complexes of sheaves $(k \geqslant 0)$ on $X$. Assume that

(i) for all $k, \Omega^{*}(k) \rightarrow \Omega^{*}(k+1)$ induces an isomorphism of cohomology sheaves,

(ii) there is a function $k \mapsto f(k)$ such that

$$
H^{q}\left(X, \Omega^{p}(k)\right) \rightarrow H^{q}\left(X, \Omega^{p}(f(k))\right)
$$

is zero for $q \geqslant 1$ and all $p_{3}$

(iii) $H^{q}\left(X, \Omega^{p}(k)\right)=0$ for $q>n$ and all $p, k$ and $\Omega^{p}(k)=0$ for $p>n$ and all $k$. Then

(a) the maps of hypercohomology

$$
\mathcal{H}^{\alpha}\left(X, \Omega^{*}(k)\right) \rightarrow \mathcal{H}^{a}\left(X, \Omega^{*}(k+1)\right)
$$

are all isomorphisms

(b) the natural homomorphism

$$
\varphi_{N}^{q}: D R^{q}\left(\Omega^{*}(N)\right) \rightarrow \mathcal{H}^{q}=\mathcal{H}^{q}\left(X, \Omega^{*}(N)\right)
$$

is surjective provided $N \geqslant f^{n}(0)$ (where $f^{n}$ denotes the $n$-th iterate fofo ... of)

(c) the kernel of $\varphi_{N}^{q}$ coincides with the kernel of $D R^{q}\left(\Omega^{*}(N)\right) \rightarrow D R^{q}\left(\Omega^{*}(M)\right)$ provided $M \geqslant f^{n-1}(N)$. 
Proof. (a) follows from (i) and (2.2). Now consider the spectral sequence $F_{r}^{p, q}(k) \Rightarrow \mathcal{H}$. We have (using (iii))

$$
\begin{aligned}
F_{1}^{p, q}(k) & =H^{q}\left(X, \Omega^{p}(k)\right) \\
F_{n+1}^{p, q}(k) & =F_{\infty}^{p, q}(k)=\mathcal{H}_{p}^{p+q}(k) / \mathcal{H}_{p+1}^{p+q}(k)
\end{aligned}
$$

where $\mathcal{H}^{s}=\mathcal{H}^{s}(k)$ is filtered

$$
\boldsymbol{H}^{s}=\boldsymbol{H}_{0}^{s}(k) \supset \boldsymbol{H}_{1}^{s}(k) \supset \ldots \supset \mathcal{H}_{s}^{s}(k) \supset \mathcal{H}_{s+1}^{s}(k)=0 .
$$

By (ii) $F_{1}^{p, q}(k) \rightarrow F_{1}^{p, q}(f(k))$ is zero for $q \geqslant 1$, and hence the same is true for $F_{\infty}^{p . q}$. Thus

$$
\mathcal{H}_{p}^{p+q}(k) \subset \mathcal{H}_{p+1}^{p+q}(f(k)) \text { for } q \geqslant 1 .
$$

Iterating this we see that,

$$
\mathcal{H}^{s}=\mathcal{H}_{0}^{s}(k) \subset \mathcal{H}_{1}^{s}(f(k)) \subset \ldots \subset \mathcal{H}_{s}^{s}\left(f^{s}(k)\right) \subset \mathcal{H}^{s}
$$

and so $\mathcal{H}_{s}^{s}\left(f^{s}(k)\right)=\mathcal{H}^{s}$. In other words the filtration on $\mathcal{H}^{s}\left(f^{s}(k)\right)$ consists of just one term, the last. The same is then true for all integers $>f^{s}(k)$. Thus for all $N \geqslant f^{n}(0)$ we have a surjection

proving (b).

$$
D R^{p}\left(\Omega^{*}(N)\right)=F_{2}^{p, 0}(n) \rightarrow F_{\infty}^{p, 0}(N)=\mathcal{H}_{p}^{p}(N)=\mathcal{H}^{p}(N)
$$

The proof of (c) proceeds in a similar fashion. Since we shall not need (c) in our applications the details will be left to the reader.

\section{§ 3. Algebraic Geometry}

We begin by recalling some basic results of Serre [17]. We consider algebraic varieties $X$ defined over $\mathbf{C}$. Then $X$ is endowed with a sheaf $o$ : for each $x \in X, o_{x}$ is a local ring-the regular functions near $x$. A sheaf $S$ of $o$-modules is called an algebraic sheaf: it is called coherent if it is locally the cokernel of a homomorphism $\boldsymbol{o}^{\mathfrak{p}} \rightarrow \boldsymbol{o}^{\alpha}$ (where $\mathfrak{o}^{\mathfrak{p}}=\mathfrak{o} \oplus \ldots \oplus \mathfrak{o}$ $p$ times). For coherent algebraic sheaves Serre defines the cohomology groups $H^{a}(X, S)$ as Cech groups but it has since been shown by Grothendieck that this coincides with the injective resolution definition. Serre proves (p. 239)

*(3.1) If $X$ is affine and $S$ is coherent then

and more generally

$$
H^{q}(X, S)=0 \text { for all } q \geqslant 1
$$

(3.2) If $U$ is a covering of $X$ by affine open sets and $S$ is coherent then,

$$
H^{q}(X, S)=H^{q}\left(C^{*}(\mathcal{U}, S)\right) \text {. }
$$

In other words we do not need to take limits over all coverings. 
For projective varieties on the other hand Serre proves (p. 259)

(3.3) $X$ projective and $S$ coherent $\Rightarrow \operatorname{dim}_{\mathrm{c}} H^{q}(X, S)<\infty$ for all $q$.

Now we can also regard $X$ as a complex analytic space (1). As such we shall write $X^{h}$ ( $h$ for holomorphic) and $o^{h}$ for the sheaf of local holomorphic functions. For any o-module $S$ we define $S^{h}=S \otimes_{\mathcal{o}} o^{h}$ (using the natural map $o \longrightarrow o^{h}$ ). Then the basic comparison theorems of Serre [19] pp. 17, 19) are

(3.4) If $S$ is a coherent algebraic sheaf on $X$ then $S^{h}$ is a coherent analytic sheaf on $X^{h}$

(3.5) If $X$ is projective algebraic and $S$ is coherent algebraic then the natural homomorphism

$$
H^{q}(X, S) \rightarrow H^{a}\left(X^{h}, S^{h}\right)
$$

is an isomorphism for all $q$.

We now come to the vanishing theorems. If $Y$ is a closed subvariety of $X$ given locally by one equation $\left({ }^{2}\right)$, denote by $J$ its sheaf of ideals. The sheaf $o(n Y)=\operatorname{Hom}_{\mathfrak{o}}\left(J^{n}, o\right)$ is the sheaf of functions on $X$ with poles of order $\leqslant n$ on $Y$. For any coherent sheaf $S$ on $X$ we put $S(n)=S(n Y)=S \otimes_{\mathfrak{o}} \mathfrak{o}(n Y)$. Then Serre's vanishing theorem asserts (Serre [19], p. 259)

(3.6) If $X$ is projective and $Y$ is a hyperplane section of $X$, then, for all $q \geqslant 1$,

$$
H^{q}(X, S(n Y))=0 \text { for all large } n \text {. }
$$

The analogous analytic theorem with $X^{h}$ replacing $X$ is the Kodaira vanishing theorem.

A slight variant of (3.6) which we shall call the Grothendieck vanishing theorem can be deduced quite easily from (3.1)-(3.3). This is as follows

Proposition (3.7). If $X$ is projective and $X-Y$ is affine then for $q \geqslant 1$ and any integer $n$ there exists $m>n$ such that

is zero.

$$
H^{q}(X, S(n)) \rightarrow H^{q}(X, S(m))
$$

Proof. $\left({ }^{3}\right)$ In view of (3.3) it will be enough to show that $\lim H^{q}(X, S(n))=0$ for all $q \geqslant 1$. Now for any affine open set $U$ of $X$ in which $Y$ is given by $f=0$ and with $U_{f}=U-U \cap Y$ we have

(1) In fact we shall always take cohomology on non-singular $X$ so that $X^{h}$ is a complex manifold.

(2) If $X$ is non-singular, this simply means $Y$ is of codimension one: then $J$ is locally free of rank one.

( $\left.{ }^{3}\right)$ This proof is extracted from footnote 6 of Grothendieck [12]. 


$$
\lim _{\rightarrow} \Gamma(U, S(n)) \cong \Gamma(U, S)_{f} \cong \Gamma\left(U_{f}, S \mid U_{f}\right)
$$

where the second term is the module of frartions with respect to all powers of $f$ : in fact, $\Gamma$ commutes with formation of fractions. Hence for any finite covering $\mathcal{U}$ of $\boldsymbol{X}$ by affine open sets we have $\left(^{(1)}\right.$

$$
\lim _{\rightarrow} H^{q}\left(C ^ { * } ( U , S ( n ) ) \cong H ^ { q } \left(\lim _{\rightarrow} C^{*}(\mathcal{U}, S(n)) \cong H^{q}\left(C^{*}(\vartheta, S \mid X-Y)\right)\right.\right.
$$

where $\mathcal{V}$ is the affine covering of $X-Y$ induced by $\mathcal{U}$. Using (3.2) this gives

$$
\lim _{\rightarrow} H^{q}(X, S(n)) \cong H^{q}(X-Y, S \mid X-Y)
$$

and. since $X-Y$ is affine, the result now follows by applying (3.1).

Proposition (3.7) is one which we shall later need to strengthen to obtain uniform bounds when $X$ depends algebraically on a parameter. As indicated in $\S 1$ this necessitates introducing Grothendieck's generalizations of Serre's theorems to the framework of schemes. We shall now review briefly what we need.

We shall consider an affine ground scheme $T=\operatorname{Spec} A$ where $A$ is a Noetherian ring (in application $A$ will be the coordinate riug of an affine variety over $\mathbf{C}$ ) and schemes $X$ over $T$. The analogues of (3.1) and (3.2) for schemes hold quite generally and (3.3) generalizes to

\section{(3.8) $X$ proper $\left({ }^{2}\right)$ over $T$ and $S$ coherent on $X \Rightarrow H^{q}(X, S)$ is a finite $A$-module for all $q$.}

Replacing (3.3) by (3.8) the proof of (3.7) goes over as it stands to schemes over $T$, the hypotheses being now that $X \rightarrow T$ is a projestive morphism and that $X-Y$ is affine. There are analogues of (3.5) but these (due to Grauert-Remmert) are more difficult and we will avoid them.

We shall also need to use results telling us what happens to sheaf cohomology under "restriction to a fibre" $X_{t}$ of $X \rightarrow T$. Recall first that $t \in T=\operatorname{Spec} A$ corresponds to a prime ideal say $p_{t}$ of $A$ and $X_{t}$ has structure sheaf $o_{t}=o / p_{t} \circ$. For any sheaf $S$ of $o$-modules on $X$ we define $S_{t}=S \otimes_{0} o_{t}=S / p_{t} S$ to be its restriction to the fibre $X_{t}$. We have a natural restriction homomorphism of $A$-modules

$$
H^{q}(X, S) \rightarrow H^{q}\left(X_{t}, S_{t}\right)
$$

inducing a homomorphism of $k(t)$-modules

$$
H^{q}(X, S) \otimes_{A} k(t) \rightarrow H^{q}\left(X_{t}, S_{t}\right)
$$

(1) Recall that the intersection of affines is affine (Serre [17]).

(2) Proper is the analogue of complete and is more general than projective. In fact we shall only use the projective case. 
where $k(t)$ is the residue field of the prime ideal $p_{t}$. The following criterion of Grothendieck [11] Cor. (6.9.10)]) gives conditions under which (3.9) is an isomorphism:

(3.10) If $S$ and all $H^{q}(X, S)$ are $A$-flat $\left(^{1}\right)$ then (3.9) is an isomorphism.

For the convenience of the reader we shall briefly indicate how (3.10) is proved. Take a resolution

$$
D^{*}: \rightarrow D_{-2} \rightarrow D_{-1} \rightarrow D_{0} \rightarrow k(t) \rightarrow 0
$$

of $k(t)$ by finite free $A$-modules $D_{i}$ and let $\mathcal{U}$ be a finite covering of $X$ by affine open sets. We then consider the hypercohomology $\mathcal{H}$ of the double complex $C^{*}\left(\mathcal{U}, S \otimes_{A} D^{*}\right)$. Since $S$ and $H^{*}(X, S)$ are both $A$-flat both spectral sequences $\left({ }^{2}\right)$ are trivial. Equating the two $E_{2}$ terms (and using (3.2) for schemes) (3.9) follows.

Combining (3.10), (3.8) and (3.7) (for schemes) we shall now prove a version of (3.7) with parameters.

Proposition (3.11). Let $T=\operatorname{Spec} A$ with $A$ Noetherian, $\pi: X \rightarrow T$ a projective morphism, $Y$ a closed subscheme of $X$ whose sheaf of ideals is principal, $S$ a coherent sheaf on $X$. Assume that all $S(m)$ are $\left({ }^{3}\right)$ A-flat and that $X-Y$ is affine, then for any $n$ there exists $m \geqslant n$ such that

is zero for $q \geqslant 1$ and all $t \in T$.

$$
H^{q}\left(X_{t}, S(n)_{t}\right) \rightarrow H^{q}\left(X_{t}, S(m)_{t}\right)
$$

Proof. Since $A$ is Noetherian the closed subsets of $T$ satisfy the descending chain condition. Using induction on the closed subsets of $T$ it will therefore be sufficient (for given $n$ ) to prove the proposition with $T$ replaced by some non-empty open subset $U$. Moreover we may assume $T$ reduced (that is $A$ has no nilpotent elements except 0 ). Now applying (3.7) for schemes we get an integer $m$ such that

$$
H^{q}(X, S(n)) \rightarrow H^{q}(X, S(m))
$$

is zero for all $q \geqslant 1$. By (3.8) both these $H^{q}$ are finite $A$-modules; hence, $\left({ }^{4}\right)$ after tensoring with a suitable ring of fractions $B$ (of the form $A_{f}$ ), they become free. Thus if we replace $T$ by $U=\operatorname{spec} B$, and recall that $H^{q}$ commutes with formation of fractions, we may assume

(1) Recall that an $A$-module $M$ is called $A$-flat if $\otimes_{A} M$ is an exact functor.

(2) These are

$$
\begin{aligned}
H^{p}\left(X, \operatorname{tor}_{-q}^{A}(S, k(t))\right. & \rightarrow \mathcal{H} \\
\operatorname{Tor}_{-Q}^{A}\left(H^{q}(X, S), k(t)\right) & \rightarrow \mathcal{H} .
\end{aligned}
$$
and

( ${ }^{3}$ This assumption is made in order to simplify the proof and is adequate for our purposes. By appealing to further results of Grothendieck it could, in fact, be dropped.

(4) This uses the fact that $T$ is reduced. 
that the modules in (3.12) are free (and therefore flat). By (3.10) therefore we have isomorphisms

$$
\begin{aligned}
& H^{q}(X, S(n)) \otimes k(t) \cong H^{q}\left(X_{t}, S(n)_{t}\right) \\
& H^{q}(X, S(m)) \otimes k(t) \cong H^{q}\left(X_{t}, S(m)_{t}\right)
\end{aligned}
$$

for all $t$. Since (3.12) is the zero homomorphism (for $q \geqslant 1$ ) the same is true for

completing the proof.

$$
H^{\alpha}\left(X_{t}, S(n)_{t}\right) \rightarrow H^{q}\left(X_{t}, S(m)_{t}\right)
$$

Remark. In application $\pi$ will be flat and $S$ will be locally free. Then all $S(m)$ are locally free and hence also $A$-flat.

Finally, in this section on Algebraic Geometry, we come to the major theorem of Hironaka [14] on the Resolution of Singularities. We shall state this in the form we require:

Resolution TheORem. Let $X$ be an algebraic variety over $\mathbf{C}, Y$ a closed subvariety with $X-Y$ non-singular. Then there exists a proper (even projective) morphism $\pi$ : $X^{\prime} \rightarrow X$ with $X^{\prime}$ non-singular such that

(i) $Y^{\prime}=\pi^{-1}(Y)$ is a finite union of non-singular submanifolds of codimension one with normal crossings

(ii) $X^{\prime}-Y^{\prime} \rightarrow Y-Y$ is an isomorphism.

We recall that $Y=\cup Y_{i}$ has normal crossings if in local analytic coordinates $\left(z_{1}, \ldots, z_{n}\right)$ its equation is given by $z_{1} z_{2} \ldots z_{r}=0$ for some $r,(1 \leqslant r \leqslant n)$.

\section{§ 4. The Algebraic de Rham Theorem}

We begin by considering the extension of the holomorphic Poincaré Lemma to allow for poles on a subvariety with normal crossings. So let $Y \subset \mathbf{C}^{n}$ be given by $z_{1} z_{2} \ldots z_{r}=0$ and let $\Omega^{q}(k)\left(=\Omega_{n_{,},}^{q}(k)\right)$ denote the germ at 0 of meromorphic $q$-forms on $\mathbf{C}^{n}$ which have poles on $Y$ of total order $\leqslant q+k$, i.e.

$$
\varphi=\sum_{|\mu| \leqslant a+k} z_{1}^{-\mu_{1}} z_{2}^{-\mu_{2}} \ldots z_{r}^{-\mu_{r}} \varphi_{\mu}
$$

where $\varphi_{\mu}$ is a holomorphic $q$-form, $\mu=\left(\mu_{1}, \ldots, \mu_{r}\right)$ and $|\mu|=\Sigma \mu_{l}$. Clearly $d \Omega^{q}(k) \subset \Omega^{a+1}(k)$ so that we have a complex

$$
\Omega^{*}(k): \Omega^{0}(k) \stackrel{d}{\rightarrow} \Omega^{1}(k) \stackrel{d}{\longrightarrow} \ldots \rightarrow \Omega^{n}(k) .
$$


Then we have $\left(^{1}\right)$

Lемма (4.1). $H^{*}\left(\Omega^{*}(k)\right)$ is an exterior algebra generated by $d z_{i} / z_{i}(i=1, \ldots, r)$, and is isomorphic to $H^{*}\left(\mathbf{C}^{n}-Y\right)$.

Proof. Let $\varphi \in \Omega^{q}(k)$ with $d \varphi=0$. If $q=0$ then $\varphi=$ constant. If $q \geqslant 1$, writing $\varphi$ in the form $\varphi=d z_{1} \alpha+\beta$ and expanding in terms of $z_{1}$,

$$
\begin{aligned}
& \alpha=\alpha_{0}+\alpha_{1} z_{1}^{-1}+\alpha_{2} z_{1}^{-2}+\ldots+\alpha_{r} z_{1}^{-r} \\
& \beta=\beta_{0}+\beta_{1} z_{1}^{-1}+\beta_{2} z_{1}^{-2}+\ldots+\beta_{\mathrm{r}} z_{1}^{-r}
\end{aligned}
$$

$\left(\alpha_{0}, \beta_{0}\right)$ holomorphic in $\left.z_{1}\right)$ we have

$$
\begin{aligned}
& d \alpha_{1}=d \alpha_{2}+\beta_{1}=d \alpha_{3}+2 \beta_{2}=\ldots=r \beta_{r}=0 \\
& d \beta_{1}=d \beta_{2}=\ldots=d \beta_{r}=0 \\
& d \varphi_{0}=0 \quad \text { where } \varphi_{0}=d z_{1} \alpha_{0}+\beta_{0} .
\end{aligned}
$$

Put $\theta=-\alpha_{2} z_{1}^{-1}-\alpha_{3}\left(2 z_{1}^{2}\right)^{-1}-\ldots-\alpha_{r}\left((r-1) z_{1}^{r-1}\right)^{-1} \in \Omega^{q-1}(k)$. Then

$$
\varphi=z_{1}^{-1} d z_{1} \alpha_{1}+\varphi_{0}+d \theta
$$

Here $\alpha_{1} \in \Omega_{n-1, r-1}^{q-1}(k)$ is independent of $z_{1}$ and $\varphi_{0} \in \Omega_{n, r-1}^{q}(k)$ has no pole on $z_{1}=0$. Since $d \alpha_{1}=d \varphi_{0}=0$ induction on $r$ and the usual Poincaré Lemma proves that

$$
\varphi=\sum c_{i_{1} i_{2} \ldots i_{q}}\left(z_{i_{1}}\right)^{-1} d z_{i_{2}} \ldots\left(z_{i_{q}}\right)^{-1} d z_{i_{q}}+d \psi
$$

where $1 \leqslant i_{1}<\ldots<i_{q} \leqslant r, c_{i_{2} \ldots i_{q}} \in \mathbf{C}$ and $\psi \in \Omega^{q-1}(k)$. This proves that $H^{*}(\Omega(k))$ is generated by the $d z_{i} / z_{i}, i=1, \ldots, r$. On the other hand, $H^{*}\left(C^{n}-Y\right)=H^{*}(B-B \cap Y$ ) (for any small ball $B$ around 0 ) is an exterior algebra on $r$ generators $€ H^{1}$ dual to the $r$ generators $€ H_{1}$ given by small circles $\gamma_{i}$ around the hyperplanes $z_{i}=0,(i=1, \ldots, r)$. Since $\int_{\gamma_{i}} z_{i}^{-1} d z_{i}=2 \pi i \delta_{i j}$ this proves that

$$
H^{*}\left(\Omega^{*}(k)\right) \rightarrow H^{*}\left(\mathbf{C}^{n}-Y\right)
$$

is then an isomorphism and completes the proof.

We can now prove the first version of the algebraic de Rham theorem.

Proposition (4.2). Let $X$ be a non-singular projective variety, $Y$ a closed subvariety of codimension one which is a finite union of non-singular subvarieties $Y_{i}$ with normal crossings, and such that $X-Y$ is affine. Then $H^{*}\left(X^{h}-Y^{h}\right)$ is isomorphic to the de Rham group of the complex of rational differential forms on $X$ with poles on $Y$.

(1) This is essentially Lemma 17 of Atiyah-Hodge [3] with attention paid to the order of pole. 
Actually since we are also interested in bounds for the orders of pole, we shall prove a more precise version of (4.2). Let us denote by $\Omega^{p}(k)$ the algebraic sheaf of $p$-forms on $X$ with pole of total order $\leqslant p+k$ on $Y$. It is easy to see that these are coherent sheaves (there are obvious finite sets of generators and relations) and we have $d \Omega^{p}(k) \subset \Omega^{p+1}(k)$. Since $X-Y$ is affine the Grothendieck vanishing theorem (3.7) applies and shows that there is a function $k \mapsto f(k)$ such that

$$
H^{q}\left(X, \Omega^{p}(k)\right) \rightarrow H^{q}\left(X, \Omega^{p}(f(k))\right)
$$

is zero for $q \geqslant 1$ and all $p, k$. By the Serre comparison theorem (3.5) we can replace $X, \Omega^{p}(k)$ here by $X^{h}, \Omega^{p}(k)^{h}$, their holomorphic counterparts. Applying (4.1) and (2.10) to the sequence of complexes $\Omega^{*}(k)$ we deduce

$$
\operatorname{Im}\left[D R^{q}\left(\Omega^{*}(N)^{h}\right) \rightarrow D R^{q}\left(\Omega^{*}(M)\right)^{h}\right] \rightarrow \mathcal{H}^{q}\left(X^{h}, \Omega^{*}(0)^{h}\right)
$$

is an isomorphism for $N \geqslant f^{n}(0), M \geqslant f^{n-1}(N)$. By Serre's Theorem (3.5) (applied to $H^{0}$ ) we see that the superscript $h$ can be removed from the de Rham groups and hence, if we now show that

$$
\mathcal{H}^{q}\left(X^{h}, \Omega^{*}(0)^{h}\right) \cong H^{q}\left(X^{h}-Y^{h}\right)
$$

then (4.2) will have been proved with bounds, namely we only need poles of total order $N$ to represent $H^{a}\left(X^{h}-Y^{h}\right)$ and all equivalences then come from poles of total order $M$.

To prove (4.3) we introduce the sheaves $\tilde{\Omega}^{q}$ of $C^{\infty} q$-forms on $X^{h}-Y^{h}$ and their direct images $i_{*}\left(\tilde{\Omega}^{q}\right)$ on $X^{n}$. Then

$$
\begin{aligned}
H^{q}\left(X^{h}-Y^{h}\right) & \cong D R^{q}\left(\tilde{\Omega}^{*}\right) \text { by } C^{\infty} \text { de Rham theorem on } X^{h}-Y^{h} \\
& =H^{q}\left(\Gamma\left(X^{h}-Y^{h}, \tilde{\Omega^{*}}\right)\right) \cong H^{q}\left(\Gamma\left(X^{h}, i_{*}\left(\Omega^{*}\right)\right) \text { by definition of } i_{*}\right. \\
& \left.\cong \mathcal{H}^{q}\left(X^{h}, i_{*}\left(\tilde{\Omega}^{*}\right)\right) \text { by }(2.8) \text { and the fact that } i_{*}\left(\tilde{\Omega}^{p}\right) \text { is fine }{ }^{1}\right) \\
& \cong \mathcal{H}^{q}\left(X^{h}, \Omega^{*}(0)^{h}\right) \text { by }(4.1) \text { and }(2.9)
\end{aligned}
$$

proving (4.3) and so completing the proof of (4.2) and the stronger version with bounds namely

Proposition (4.2)'. Let $X, Y$ be as in (4.2) and let $k \mapsto f(k)$ be a function such that

$$
H^{q}\left(X, \Omega^{p}(k)\right) \rightarrow H^{q}\left(X, \Omega^{p}(f(k))\right)
$$

is zero for $q \geqslant 1$ and all $p$. Then

$$
\operatorname{Im}\left[D R^{q}\left(\Omega^{*}(N)\right) \rightarrow D R^{\alpha}\left(\Omega^{*}(M)\right)\right] \rightarrow H^{\alpha}\left(X^{h}-Y^{h}\right)
$$

is an isomorphism for all $q$, provided $N \geqslant f^{n}(0)$ and $M \geqslant f^{n-1}(N)$.

(1) Being a module over $C^{\infty}(X)$. 
To deduce the general case from (4.2) we now appeal to the Resolution of Singularities. Given an affine non-singular variety ${ }^{(1)} X \subset \mathbf{C}^{N}$ let $\bar{X}$ be its closure in $P_{N}, Y=\bar{X}-X$ and apply the Resolution Theorem. We get $\pi: \bar{X}^{\prime} \rightarrow \bar{X}$ proper with $\bar{X}^{\prime}$ non-singular, $Y^{\prime}=\pi^{-1}(Y)$ a finite union of non-singular subvarieties of codimension one with normal crossings, and $\bar{X}^{\prime}-Y^{\prime} \cong X$. Applying (4.2) to $X^{\prime}, Y^{\prime}$ we deduce

THEOREM (4.4). For any non-singular affine variety $X$ the cohomology groups $H^{q}\left(X^{h}\right)$ are isomorphic to the de Rham groups of the rational differential forms on $X$ with poles at $\infty$.

We want now to show that, for $X$ in an algebraic family, we can put a bound on the order of poles needed. For our purposes the following version will be adequate.

THEOREM (4.5). Let $\pi: X \rightarrow T$ be a smooth $\left({ }^{2}\right)$ projective morphism of non-singular algebraic varieties and let $Y$ be a subvariety of $X$ of codimension one such that $X-Y$ is affine. Then there exists an integer $L$ so that, for all $t \in T$, the rational closed forms on $X_{t}$ with poles of order $\leqslant L$ on $Y_{t}$ generate $\left(^{3}\right) H^{*}\left(X_{t}^{h}-Y_{t}^{h}\right)$, where $X_{t}=\pi^{-1}(t), Y_{t}=X_{t} \cap Y$.

Proof. We shall use induction on $\operatorname{dim} T$. This will enable us to replace $T$ at any stage by a (Zariski) open subset since $\left(^{4}\right)$ the complement then has lower dimension (and is a finite union of non-singular pieces). Now resolve the singularities of $Y$ and we obtain $p: X^{\prime}, Y^{\prime} \rightarrow X, Y, \quad Y^{\prime}$ having normal crossings. Replacing $T$ by an open subset we may assume that $X^{\prime} \rightarrow T$ and all $Y_{i_{1}}^{\prime} \cap Y_{i_{2}}^{\prime} \cap \ldots \cap Y_{i_{s}}^{\prime} \rightarrow T$ are smooth, so that all $X_{t}^{\prime}$ are nonsingular and all $Y_{t}^{\prime}$ have normal crossings. If $J, J^{\prime}$ are the ideals of $Y, Y^{\prime}$ respectively, then $\left(J^{\prime}\right)^{m} \subset p^{*} J \subset J^{\prime}$ for some $m$, hence $\left(J_{t}^{\prime}\right)^{m} \subset p^{*} J_{t} \subset J_{t}^{\prime}$ for all $t$, so that a bound $L^{\prime}$ for $X^{\prime}, Y^{\prime}$ will give a bound $L=m L^{\prime}$ for $X, Y$. Hence it is enough to prove the theorem for $X^{\prime}, Y^{\prime}$.

For each $t$ consider now the sheaf $\Omega_{t}^{q}(k)$ of $q$-forms on $X_{t}^{\prime}$ having a pole of total order $\leqslant q+k$ on $Y_{t}^{\prime}$. Consider also the sheaf $\Omega^{q}\left(k Y_{t}^{\prime}\right)$ of $q$-forms on $X_{t}^{\prime}$ having poles of order $\leqslant q+k$ on each component of $Y_{t}^{\prime}$. This is a locally free sheaf and (because $X^{\prime} \rightarrow T$ is smooth) is also the restriction to $X_{t}^{\prime}$ of a locally free sheaf, say $S^{q}(k)$ on $X^{\prime}$, so that $\Omega^{q}\left(k Y_{t}^{\prime}\right)=$ $S^{q}(k)_{t}$. In fact $S^{q}(k)$ is the sheaf of $q$-forms on $X^{\prime}$ with poles of order $\leqslant q+k$ on $Y^{\prime}$. Moreover this is consistent with our earlier notation used in (3.11), namely $S^{a}(k)$ is in fact $S^{q}$ with poles of order $k$ on $Y$, where $S^{q}=S^{q}(0)$. Applying (3.11) therefore $\left(^{5}\right.$ ) (permissible since smooth $\Leftarrow$ flat) and using the obvious inclusions

(1) We now make a slight change of notation more natural to the affine situation.

(2) I.e. Jacobian $\pi$ has maximal $\operatorname{rank}=\operatorname{dim} T$.

$\left.{ }^{3}\right)$ The other bound for the relations (as in (4.2)') also holds but we do not require it.

(4) At least if $T$ is irreducible which we may clearly assume since in general $T$ is a finite union of such.

$\left.{ }^{5}\right)$ See also the Remark after (3.11). 


$$
\Omega_{t}^{q}(k) \subset \Omega^{q}\left(k Y^{\prime}\right)_{t} \subset \Omega_{t}^{q}(n(q+k))
$$

we obtain a function $k \mapsto f(k)$ so that for $q \geqslant 1, t \in T$ and all $p$

$$
H^{q}\left(X_{t}^{\prime}, \Omega_{t}^{p}(k)\right) \rightarrow H^{q}\left(X_{t}^{\prime}, \Omega_{t}^{p}(n(q+f(k))\right.
$$

is zero. Now apply (4.2)' and the proof is complete.

We shall now consider a mild extension of Theorem (4.4). We suppose $X$ affine non-singular and $Y \subset X$ a codimension one subvariety with normal crossings. $\left({ }^{1}\right)$. Then $X-Y$ is again affine so that its cohomology is, by (4.4), given by the rational differentials on $X$ with poles on $Y$ (and "at $\infty$ "). However we shall now show that only simple poles on the components $Y_{i}$ of $Y$ are required-though we still need high order poles at $\infty$. We introduce the sheaves $\Omega^{a}\langle Y\rangle$ on $X$ of rational $q$-forms $\omega$ such that $\omega$ and $d \omega$ have only simple poles on each $Y_{i}$. These form a complex $\Omega^{*}\langle Y\rangle$ :

$$
0 \rightarrow \Omega^{0}\langle Y\rangle \rightarrow \ldots \rightarrow \Omega^{q}\langle Y\rangle \stackrel{d}{\rightarrow} \ldots
$$

and Theorem (4.4) is refined by

THEOR EM (4.6). If $X$ is a non-singular affine variety, $Y$ a codimension one subvariety with normal crossings, then the cohomology groups $H^{q}\left((X-Y)^{h}\right)$ are isomorphic to the de Rham groups of the complex $\Gamma\left(X, \Omega^{*}\langle Y\rangle\right)$ of rational differentials $\omega$ such that $\omega$ and $d \omega$ have only simple poles on the components of $Y$.

The proof of (4.6) follows the same lines as (4.4) and we shall just comment on the differences. First we must use the resolution theorem to obtain a compactification $\bar{X}$ of $X$ so that not only $Z=\bar{X}-X$ has normal crossings, but also $Z \cup Y$ has only normal crossings. Next we must observe that the sheaves $\Omega^{\alpha}\langle Y\rangle$ are algebraic: the equation

$$
d(g \omega)=d g \wedge \omega+g d \omega
$$

shows that sections of $\Omega^{q}\langle Y\rangle$ can be multiplied by regular functions. If $\left(z_{1}, \ldots, z_{n}\right)$ is a system of local parameters on $X$ with $Y$ given by $z_{1} z_{2} \ldots z_{s}=0$ then the sections of $\Omega^{*}\langle Y\rangle$ are generated (as an exterior algebra over the functions) by $d z_{i} / z_{i}(1 \leqslant i \leqslant s)$ and $d z_{j}(j>s)$. Similar remarks apply to the sheaves on $\bar{X}$ of $q$-forms $\omega$ such that $\omega, d \omega$ have simple poles on the $Y_{i}$ and such that $\omega$ has on $Z \cup Y$ a pole of total order $\leqslant q+k$. Finally we must modify Lemma (4.1), by replacing the complex $\Omega^{*}(k)$ by the sub-complex of forms $\omega$ such that $\omega$ and $d \omega$ have only simple poles on $z_{r-s+1}=0, \ldots z_{r}=0$ : the conclusion and proof are the same.

(1) I.e. $Y=\cup Y_{t}, Y_{i}$ non-singular and meeting transversally. In fact we can drop the condition of non-singularity for the $Y_{i}$ : as the proof shows only local hypotheses on $Y$ are used. Thus $Y$ could be a curve with a double point. 
With these modifications the proof of (4.6) proceeds as before: (4.1) takes care of the local cohomology and the Grothendieck vanishing theorem still applies because we are allowed arbitrary poles on $Z$ and $X=\bar{X}-Z$ is affine.

An alternative proof of (4.6) can be given, starting from (4.4) and using some exact sequences. In the simplest case when $Y$ has only one component we use the exact sequence of sheaves on $X$

$$
0 \rightarrow \Omega^{\alpha} \rightarrow \Omega^{q}\langle Y\rangle \stackrel{R}{\rightarrow} \Omega_{Y}^{\alpha-1} \rightarrow 0
$$

where $\Omega_{Y}^{\alpha-1}$ is the sheaf of regular $(q-1)$ )-forms on $Y$ (extended by zero to $X$ ) and $R$ is the residue map which in local coordinates maps $\omega \wedge d z / z \mapsto 2 \pi i \omega \mid Y$ where $z=0$ is the local equation of $Y$. Since $X$ is affine we have $H^{1}\left(X, \Omega^{q}\right)=0$ and so (4.7) gives a corresponding exact sequence of global sections. Thus we have an exact sequence of complexes

$$
0 \rightarrow \Gamma\left(X, \Omega^{*}\right) \rightarrow \Gamma\left(X, \Omega^{*}\langle Y\rangle\right) \rightarrow \Gamma\left(Y, \Omega_{Y}^{*}\right) \rightarrow 0 .
$$

Taking the cohomology of these complexes we get a long exact sequence of de Rham groups

$$
\ldots \rightarrow D R^{q}\left(X, \Omega^{*}\right) \rightarrow D R^{q}\left(X, \Omega^{*}\langle Y\rangle\right) \rightarrow D R^{q}\left(Y, \Omega_{Y}^{*}\right) \rightarrow \ldots .
$$

We now map this into the exact cohomology sequence of the pair $\left(X^{h},(X-Y)^{n}\right)$ using the Thom isomorphism

$$
H^{q}\left(X^{h},(X-Y)^{h}\right) \cong H^{q-2}\left(Y^{h}\right) .
$$

It is not difficult to verify commutativity of the diagram. Applying (4.4) to $X$ and $Y$ we get isomorphisms for two-thirds of the terms and hence, by the 5-Lemma, isomorphisms also for the other groups, namely

$$
D R^{q}\left(X, \Omega^{*}\langle Y\rangle \cong H^{q}\left((X-Y)^{h}\right)\right.
$$

proving (4.6), for this case. In general the proof proceeds the same way but using induction on the number of components of $Y$.

\section{§ 5. Complements of hypersurfaces}

For the applications to lacunas the algebraic varieties which enter are of the form ( $\left.{ }^{1}\right)$ $X=P_{n-1}-A$ where $A$ is a hypersurface in projective space $P_{n-1}$, given by an equation $a\left(\xi_{1}, \ldots, \xi_{n}\right)=0$ (a being homogeneous of degree $m>0$ ). Let us first observe that such an $X$ is in fact affine. To see this we use the Veronese embedding $s: P_{n-1} \rightarrow P_{N}$ given by $\xi \mapsto \xi^{\alpha}$ where $\alpha$ runs through all $n$-tuples $\left(\alpha_{1}, \ldots, \alpha_{n}\right)$ with $\sum \alpha_{i}=m$. If $a(\xi)=\sum a_{\alpha} \xi^{\alpha}$ we see that $s(X)$ is the part of $s\left(P_{n-1}\right)$ lying in the affine space $\mathbf{C}^{N} \subset P_{N}$ where $\Sigma a_{\alpha} \omega_{\alpha} \neq \mathbf{0}\left(\omega_{\alpha}\right.$ being the coordinates of $\left.P_{N}\right)$. Thus $X$ is affine and $A$ is a hyperplane section of $s\left(P_{n-1}\right) \cong P_{n-1}$.

(1) From now on we shall drop the distinction between $X$ and $X^{h}$. 
We consider next the algebraic differential forms on $X$. These are just the rational differential forms on $P_{n-1}$ with poles on $A$. To write these explicitly in homogeneous coordinates we observe that a differential form $\varphi$ on $P_{n-1}$ lifts to a differential form $\pi^{*}(\varphi)$ on $\mathbf{C}^{n}-0$ and is characterized there by the two properties

(i) invariance under scalar multiplication,

(ii) annihilation by interior product with the tangent along the fibre of $\pi: \mathbf{C}^{n}-0 \rightarrow P_{n-1}$.

That is, a differential form $\psi$ on $\mathbf{C}^{n}-0$ is of the form $\pi^{*}(\varphi)$ if and only if $\psi$ satisfies (i) and (ii). If $\psi$ is rational so that

$$
\psi=\sum \psi_{i_{1} \ldots i_{p}}(\xi) d \xi_{i_{1}} \wedge \ldots d \xi_{i_{p}}
$$

with the coefficients $\psi_{i_{1} \ldots i_{p}}(\xi)$ rational, condition (i) asserts that all these $\psi_{i_{1}, \ldots i_{p}}(\xi)$ are homogeneous in $\xi$ of degree $-p$, condition (ii) asserts that

$$
\left(\sum \xi_{i} \frac{\partial}{\partial \xi_{i}}\right) \cdot \psi=0
$$

where . stands here for interior multiplication. Now it is well known that the complex

$$
0 \rightarrow \wedge^{n}\left(V^{*}\right) \rightarrow \wedge^{n-1}\left(V^{*}\right) \rightarrow \ldots \rightarrow \wedge^{0}\left(V^{*}\right) \rightarrow 0
$$

is acyclic, where $V$ is a vector space of dimension $n$ over a field $k$, and the maps are given by interior multiplication by a non-zero element $u \in V$. Apply this with $k=\mathbf{C}\left(\xi_{1}, \ldots, \xi_{n}\right)$. $V=\mathbb{C}^{n} \otimes c k, u=\left(\xi_{1}, \ldots, \xi_{n}\right)$ and we see that condition (ii) is equivalent to saying that $\psi$ is in the image of interior multiplication by $\Sigma \xi_{i}\left(\partial / \partial \xi_{i}\right)$. In particular, for $p=n-1, \psi$ is a multiple of

$$
\begin{aligned}
\omega(\xi) & =\left(\sum \xi_{i} \frac{\partial}{\partial \xi_{i}}\right) d \xi_{1} \wedge \ldots \wedge d \xi_{n} \\
& =\sum(-1)^{j-1} \xi_{j} d \xi_{1} \wedge \ldots \wedge d \xi_{j-1} \wedge d \xi_{j+1} \wedge \ldots \wedge d \xi_{n}
\end{aligned}
$$

Thus the top degree rational forms on $P_{n-1}$ are of the form $f(\xi) \omega$, where $f(\xi)$ is a rational function of homogeneity $-n$. Those with poles on $A$ are therefore of the form

$$
\frac{g(\xi) \omega(\xi)}{a(\xi)^{a}}
$$

where $g(\xi)$ is a homogeneous polynomial of degree $m q-n$.

Applying the algebraic de Rham theorem (4.4) to top degree forms on $X$ we therefore deduce the special case we need. 
Proposition (5.1). Every cohomology class of dimension $n-1$ on $P_{n-1}-A$ can be represented by a differential form $g(\xi) a(\xi)^{-\alpha} \omega(\xi)$ where $g(\xi)$ is a homogeneous polynomial of degree $m q-n$ and $q$ is sufficiently large.

To get a bound for $q$ in (5.1) we must use Theorem (4.5). Consider therefore the product $X=P_{n-1} \times P_{N}$ where $P_{N}$ is the space representing all hypersurfaces of $P_{n-1}$ of degree $m$. The equation $\Sigma_{|\alpha|=m} a_{\alpha} \xi^{\alpha}=0$ then defines a codimension one subvariety $Y$ of $X$. For every $a \in P_{N}, Y_{a}=Y \cap X_{a}$ is just the hypersurface in $P_{n-1}$ with equation $a(\xi)=0$ $\left(X_{a}=P_{n-1} \times\{a\} \approx P_{n-1}\right)$. The projection $X \rightarrow P_{N}$ is of course smooth and so we can apply (4.5) to every affine submanifold $T$ of $P_{N}$. Since $P_{N}$ is a finite union of such $T$ (namely $P_{M}-P_{M-1}$ for $\left.M=N, N-1, \ldots, 1\right)$ we deduce

Proposition (5.2). There exists an integer $q_{0}(m, n)$ so that (5.1) holds for all $q \geqslant q_{0}(m, n)$.

When we make some non-singularity assumptions we can get more explicit bounds for $q$. First, from (4.6) we deduce

Proposition (5.3). Assume that $a(\xi)=0$ defines a hypersurface $A$ of $P_{n-1}$ with only normal crossings and let $B$ be any other hypersurface. Then every cohomology class of: dimension $n-1$ on $P_{n-1}-A-B$ can be represented by a differential form

$$
\frac{g(\xi) \omega(\xi)}{a(\xi) b(\xi)^{q}}
$$

where $g(\xi)$ is a homogeneous polynomial of degree $q \operatorname{deg} b+\operatorname{deg} a-n$, and $q$ is sufficiently large.

Returning to (5.1) let us now assume that $A$ is non-singular. Then we shall prove-

Proposition (5.4). If $A$ is non-singular then (5.1) holds with $q \geqslant n-1$.

Proof. Returning to the proof of (4.2) we consider the sheaf $\Omega^{p}(0)$ of algebraic $p$-forms on $X=P_{n-1}$ with a pole of order $\leqslant p$ on $A$. By a result of Bott [6] p. 228 we have a more precise vanishing theorem in this case, namely

$$
H^{q}\left(P_{n-1}^{h}, \Omega^{p}(0)^{h}\right)=0 \quad \text { for } q \geqslant 1 \text { and all } p .
$$

Hence (cf. (2.8)) $D R^{q}\left(\Omega^{*}(0)\right) \cong \mathcal{H}^{q}\left(P_{n-1}^{h}, \Omega^{*}(0)^{h}\right)$ for all $q$. On the other hand, using Lemma (4.1), with $k=0$, we see that

$$
\mathcal{H}^{q}\left(P_{n-1}^{h}, \Omega^{*}(0)^{h}\right) \cong H^{q}\left(P_{n-1}^{h}-A^{h}\right) .
$$

Thus

$$
D R^{\alpha}\left(\Omega^{*}(0)\right) \cong H^{\alpha}\left(P_{n-1}^{h}-A^{h}\right) \text {. }
$$


In particular, for $q=n-1$, we see that we only need $(n-1)$-forms with poles of order $n-1$ on $A$. This completes the proof.

Remark. Instead of using the vanishing theorem of Bott we can deduce (5.4) from (5.1) using a result of Macauley (see Griffiths [10]).

For the applications to hyperbolic systems we need to strengthen (5.1) (in the nonsingular case) by allowing the numerator $g(\xi)$ to be divisible by a pre-assigned polynomial. We shall now prove this by some elementary algebraic computations with the rational de Rham complex. As before write

$$
\tau(\xi)=d \xi_{1} \wedge \ldots \wedge d \xi_{n}, \quad \omega(\xi)=\sum \xi_{j} \tau_{j}(\xi)
$$

where $\tau_{j}(\xi)=(-1)^{j-1} d \xi_{1} \wedge \ldots \wedge d \xi_{j-1} \wedge d \xi_{j+1} \wedge \ldots \wedge d \xi_{n}$ so that $\tau(\xi)=d \xi_{j} \wedge \tau_{j}(\xi)$. Similarly let $\tau_{i j}(\xi)$ be the product of the $d \xi_{k}(k \neq i, j)$ ordered so that $\tau(\xi)=d \xi_{j} \wedge d \xi_{i} \wedge \tau_{i j}(\xi)$ and hence $\tau_{j}(\xi)=d \xi_{i} \wedge \tau_{i j}(\xi)$. Then we have

LEMMA (5.5). Let $\varphi(\xi)$ be a homogeneous rational function of degree $1-n$, then

$$
\left(\partial \varphi / \partial \xi_{i}\right) \omega=-d\left(\sum_{j \neq i} \varphi \xi_{j} \tau_{j i}\right)
$$

Proof. With $\varphi_{i}=\partial \varphi / \partial \xi_{i}$ we get

since $\sum \varphi_{j} \xi_{j}=(1-n)$.

$$
\begin{aligned}
d\left(\sum_{j \neq i} \varphi \xi_{j} \tau_{j i}\right) & =d \varphi \wedge \sum_{j \neq i} \xi_{j} \tau_{j i}+\varphi \sum_{j \neq i} d \xi_{j} \wedge \tau_{j i} \\
& =-\varphi_{i} \sum_{j \neq i} \xi_{j} \tau_{j}+\sum_{j \neq i} \varphi_{j} \xi_{j} \tau_{i}+(n-1) \varphi \tau_{i}=-\varphi_{i} \omega
\end{aligned}
$$

The $(n-2)$-form $\theta=\Sigma \varphi \xi_{j} \tau_{j i}$ is homogeneous of degree 0 and is annihilated by $D=\Sigma \tau_{j} \partial / \partial \xi_{j}$ (because $\theta=D \varphi \tau_{i}$ and $D^{2}=0$ ), hence it represents a rational $(n-2)$-form on $P_{n-1}$ with poles at the poles of $\varphi$. Thus (5.5) asserts that the form $\varphi_{i} \omega$ is cohomologous to zero in $P_{n-1}-Y$ where $Y$ is the polar set of $\varphi$. Using (5.5) and the identity

we deduce a homology

$$
\left(b^{-p} a^{1-q} f\right)_{i}=-q b^{-p} a^{-q} f a_{i}+a^{1-q}\left(b^{-p} f\right)_{i}
$$

$$
b^{-p} a^{-q} f a_{i} \omega \sim b^{-s} a^{-1} h \omega
$$

in $P_{n-1}-A-B$ ( $f$ and $h$ being homogeneous polynomials of the appropriate degrees). If $a(\xi)=0$ is the equation of a non-singular hypersurface $A$ then the equations $a_{i}(\xi)=0$ $(i=1, \ldots, n)$ have only $\xi=0$ as a solution. Hence $\xi_{i} \cdot \epsilon\left(a_{1}, \ldots, a_{n}\right)$ for some $r_{i}$ and so every homogeneous polynomial of sufficiently high degree lies in the ideal $\left(a_{1}, \ldots, a_{n}\right)$. By (5.6) this implies that all rational ( $n-1)$-forms with poles on $A \cup B$ are homologous to forms 
with simple poles on $A$ (since we may replace $b^{-p} f$ by $b^{-p-r} f b^{r}$ we can assume $f$ has large degree). This gives another proof of (5.3) for non-singular $A$ (assuming (5.1)).

Lемма (5.7). Let I be a non-zero homogeneous ideal invariant under all the operations $(a(\xi)$ being fixed $)$. Then

$$
T_{j j}: h \mapsto h_{i} a_{j}-h_{j} a_{i}
$$

(i) the radical $J$ of $I$ is also invariant under all $T_{i j}$

(ii) $a \in J$

(iii) if the $a_{j}(\xi)$ have only $\xi=0$ as common zero then $J=(a)$ or $J=\left(\xi_{1}, \ldots, \xi_{n}\right)$.

Proof. We have, for any positive integer $s$,

$$
T_{i j}^{s} h^{s} \equiv s !\left(T_{i j} h\right)^{s} \bmod h .
$$

Hence

$$
\begin{aligned}
h \in J & \Rightarrow h^{s} \in I \quad \text { for some } s \\
& \Rightarrow T_{i j}^{s} h^{s} \in I \Rightarrow\left(T_{i j} h\right)^{s} \in I \quad \text { by (1) } \Rightarrow T_{i j} h \in J \quad \text { proving (i). }
\end{aligned}
$$

Next

$$
\begin{aligned}
h \in I & \Rightarrow \sum_{j} \xi_{j}\left(h_{i} a_{j}-h_{j} a_{i}\right) \in I \\
& \Rightarrow m a h_{i}-(\operatorname{deg} h) h a_{i} \in I \Rightarrow a h_{i} \in I
\end{aligned}
$$

Iterating this and using induction on $|\alpha|$ we deduce

$$
h \in I \Rightarrow\left(\frac{\partial}{\partial \xi}\right)^{\alpha} h a^{|\alpha|} \in I
$$

for all $\alpha=\left(\alpha_{1}, \ldots, \alpha_{n}\right)$. Since $I \neq 0$, there is a $h \neq 0$ in $I$ and so for some $\alpha$ with $|\alpha|=$ $\operatorname{deg} h=s$ we deduce that $a^{s} \in I$, i.e. $a \in J$, proving (ii). Now let $V \subset P_{n-1}$ be the variety defined by $I$, so that $J$ consists of all polynomials vanishing on $V$. By (ii) we have $V \subset A$, the non-singular hypersurface with equation $a(\xi)=0$ in $P_{n-1}$. We have to prove that $V=A$ or $V=\varnothing$. We assume therefore that $V$ is a non-empty proper subvariety of $A$ and propose to derive a contradiction. Let $V=V_{1} \cup V_{2} \cup \ldots \cup V_{r}$ be the decomposition of $V$ into irreducible components and let $\eta$ be a non-singular point of $V_{1}$ not lying on any $V,(j>1)$. Then for any $h \in J$ we have, by (i), $h_{i} a_{j}-h_{j} a_{i} \in J$ and so $h_{i}(\eta) a_{j}(\eta)=h_{j}(\eta) a_{i}(\eta)$ for all $i, j$. This means geometrically that the hypersurface $h=0$ touches $A$ at the point $\eta$. We will show that this cannot be true for all $h$. Since $\eta$ is a non-singular point of $V_{1}$ we can find rational functions $\varphi^{1}, \ldots, \varphi^{n-1}$ forming a system of parameters for $P_{n-1}$ at $\eta$ and such that $V_{1}$ is given locally by $\varphi^{1}=\varphi^{2}=\ldots=\varphi^{s}=0$; here $s=\operatorname{codim} V_{1} \geqslant 2$, since $V$ is assumed a proper subvariety of $A$. Expressing the $\varphi^{i}$ in homogeneous coordinates and clearing denominators we obtain homogeneous polynomials $f^{1}, \ldots, f^{n-1}$. Since the $\varphi^{i}$ are local parameters the vectors grad $f^{i}(\eta), i=1, \ldots, s$, are independent and hence, since 
$s \geqslant 2$, for some $f^{i}=h$ we do not have $h_{j}(\eta) a_{j}(\eta)=h_{j}(\eta) a_{i}(\eta)$ for all $i, j$. Now $h$ vanishes on $V_{1}$ but may not vanish on the other components $V_{j}(j \geqslant 1)$ of $V$. However since $\eta \notin U_{j>1} V_{j}$ we can find $g$ vanishing on all $V_{j}(j>1)$ but with $g((\eta) \neq 0$. Replacing our $h$ above by $h^{\prime}=h g$ we now have a polynomial vanishing on all $V$ (and hence in $J$ ) but

$$
h_{i}^{\prime}(\eta) a_{j}(\eta)-h_{j}^{\prime}(\eta) a_{i}(\eta)=g(\eta)\left[h_{i}(\eta) a_{j}(\eta)-h_{j}(\eta) a_{i}(\eta)\right]
$$

and so does not vanish for all $i, j$. This completes the proof.

Using this lemma together with (5.5) we shall now prove the following refinement of $(5.3)$ :

Proposition (5.8). Let $a(\xi)=0, b(\xi)=0$ be the equations of the hypersurfaces $A, B$ with $A$ non-singular, and let $g(\xi)$ be any homogeneous polynomial not divisible by $a(\xi)$. Then every element of $H^{n-1}\left(P_{n-1}-A-B\right)$ can be represented by a differential form $b(\xi)^{-p} a(\xi)^{-1} f(\xi) g(\xi) \omega(\xi)$ where $p$ is sufficiently large and $f(\xi)$ is homogeneous of the appropriate degree.

Proof. Let $S_{k}$ denote the space of all homogeneous polynomials $f$ of degree $k$ such that the form $b^{-p} a^{-1} f \omega$ is homologous to one with numerator divisible by $g$; here we must have $k=p(\operatorname{deg} b)+m-n(m=\operatorname{deg} a)$. Now by (5.5) we have a homology

$$
b^{-p} a^{-1} f\left(h_{i} a_{j}-h_{j} a_{i}\right) \omega \sim-h\left(\left(b^{-p} a^{-1} f\right)_{i} a_{j}-\left(b^{-p} a^{-1} f\right)_{j} a_{i}\right) \omega=b^{-p-1} a^{-1} l h \omega .
$$

This shows that if $S=\Sigma S_{k}$ contains all multiples of $h$ (of degree $\equiv m-n \bmod \operatorname{deg} b$ ) it also contains all multiples of $T_{i j}(h)$ (of degree $\equiv m-n \bmod \operatorname{deg} b$ ). Hence, if $I=\Sigma I_{k}$ is the smallest homogeneous ideal containing $g$ and closed under all $T_{i j}$, we have $I_{k} \subset S_{k}$ for $k \equiv m-n \bmod \operatorname{deg} b$. But by (5.7) the ideal $I$ defines the variety 0 in $\mathbf{C}^{n}$ and so every polynomial of sufficiently high degree $k$ lies in $I$ and hence also in $S$ (if $k \equiv m-n \bmod \operatorname{deg} b$ ). Using (5.3) the result now follows.

Remarks. This proposition can be seen in a more general context by introducing the relative cohomology groups $H(X, U)$ for $X$ affine and $Y$ a divisor (equation $g=0$ ). It can be proved (1) that (roughly speaking $H(X, Y)$ can be computed from the rational forms on $X$ with poles on $X$ and "large" zeros on $Y$. In top dimension we have $H^{n}(Y)=0(n=\operatorname{dim} X>\operatorname{dim} Y)$ and so $H^{n}(X, Y) \rightarrow H^{n}(X)$ is surjective. Hence every class in $H^{n}(X)$ can be represented by a rational form with high zeros on $Y$ (i.e. divisible by a high power of $g$ ). This result holds quite generally but with further non-singularity restrictions at $\infty$ one can be more precise and prove in particular (5.8). We have preferred the more elementary approach adopted above because we have no application to hyperbolic systems of the more general results corresponding to singular $a(\xi)$.

(1) We are indebted to $P$. Deligne for this information: the proof uses ideas to be found in the Appendix to Hartshorne [13]. 


\section{§6. Plane curves}

In this section we shall show that, for an algebraic curve $X$ in $P_{2}$, precise results on the order of pole can be proved without any assumptions of non-singularity. Namely we will prove

THEOR EM (6.1). $H^{2}\left(P_{2}-X\right)$ is generated by the rational differentials $\varphi$ on $P_{2}$ having a simple pole on $X$ together with their complex conjugates $\bar{\varphi}$.

THEOREM (6.2). $H^{2}\left(P_{2}-X\right)$ is generated by the rational differentials on $P_{2}$ having $a$ double pole on $X$.

Remarks. (1) For non-singular $X(6.2)$ is just a special case of (5.4). Thus (6.2) asserts that the general bound $q_{0}(m, 3)$ of $(5.2)$ is equal to 2 . We do not know if the corresponding result is true for higher values of $n$-it seems unlikely but explicit counter examples seem difficult to produce.

(2) For non-singular $X(6.1)$ is rather easy to prove. It is essentially equivalent to the fact that the holomorphic 1 -forms on $X$ are all obtained as the residues of the rational 2 -forms on $P_{2}$ with a simple pole on $X$.

(3) In the applications to hyperbolic equations (6.1) is quite important, (6.2) less so.

(4) Note that (6.1) is equivalent to the assertion: $\alpha$ a real 2-cycle on $P_{2}-X$ and $\int_{\alpha} \varphi=0$ for all rational 2 -forms $\varphi$ with a simple pole on $X \Rightarrow \alpha \sim 0$.

We come now to the proof of (6.1). Consider first the diagram

$$
\begin{aligned}
& H_{\text {comp }}^{2}\left(P_{2}-X ; \mathrm{C}\right) \otimes H^{2}\left(P_{2}-X ; \mathrm{C}\right) \rightarrow H_{\text {comp }}^{4}\left(P_{2}-X ; \mathrm{C}\right) \cong \mathrm{C} \\
& \downarrow_{\alpha} \quad \uparrow^{\beta} \\
& H^{2}\left(P_{2}, \text { o }(-X)\right) \otimes H^{0}\left(P_{2}, \Omega^{2}(X)\right) \rightarrow H^{2}\left(P_{2}, \Omega^{2}\right) \cong \mathrm{C}
\end{aligned}
$$

where $o(-X)$ is the sheaf of holomorphic functions vanishing on $X, \Omega^{2}$ is the sheaf of holomorphic 2-forms and $\Omega^{2}(X)$ the 2-forms with a simple pole on $X$. The horizontal pairings are both dualities: the top row is Poincaré duality and the bottom row is Serre duality [18]. We claim that via these dualities $\alpha$ and $\beta$ are adjoints of one another. This is a routine kind of verification which we will give later. Assuming this we see that $\operatorname{Im} \beta$ and $\overline{\operatorname{Im} \beta}$ will generate $H^{2}\left(P_{2}-X ; \mathbf{C}\right) \Leftrightarrow \alpha$ is injective on $H_{\text {comp }}^{2}\left(P_{2}-X ; \mathbf{R}\right.$ ) (cf. Remark (4) above). To prove this we use the two exact sequences of sheaves on $P_{2}$

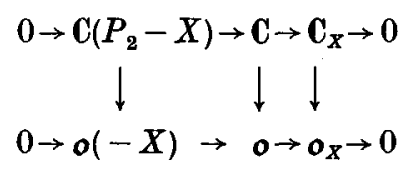


Taking cohomology we get

$$
\begin{aligned}
& 0=H^{1}\left(P_{2}, \mathbf{C}\right) \rightarrow H^{1}(X, \mathbf{C}) \rightarrow H_{\text {comp }}^{2}\left(P_{2}-X, \mathbf{C}\right) \rightarrow H^{2}\left(P_{2}, \mathbf{C}\right) \stackrel{\theta}{\rightarrow} H^{2}(X, \mathbf{C})
\end{aligned}
$$

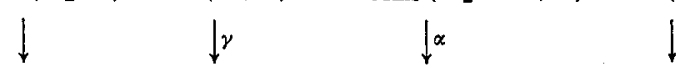

$$
\begin{aligned}
& 0=H^{1}\left(P_{2}, \text { o }\right) \rightarrow H^{1}\left(X, \text { o }_{X}\right) \rightarrow H^{2}\left(P_{2}, \text { o }(-X)\right) \rightarrow H^{2}\left(P_{2}, \mathcal{o}\right)=0
\end{aligned}
$$

where we have identified $H^{2}\left(P_{2}, \mathrm{C}\left(P_{2}-X\right)\right)$ with $H_{\text {comp }}^{2}\left(P_{2}-X\right.$, C) (see Godement [9] p. 190) and we have used the fact that $H^{q}\left(P_{2}, o\right)=0$ for $q \geqslant 1$ ([17], p. 258). Since $\theta$ is. injective (in fact isomorphic onto any irreducible component of $X$ ) the diagram reduces to a square

$$
\begin{gathered}
H^{1}(X, \mathrm{C}) \cong H_{\text {comp }}^{2}\left(P_{2}-X, \mathrm{C}\right) \\
\downarrow \gamma \\
H^{1}\left(X, o_{X}\right) \cong H^{2}\left(P_{2}, o(-X)\right)
\end{gathered}
$$

and so we are reduced to showing that $\gamma$ is injective on $H^{1}(X, \mathbf{R})$. Now $\gamma$ fits into the exact cohomology sequence

$$
H^{0}\left(X, o_{X} / \mathrm{C}\right) \stackrel{\delta}{\rightarrow} H^{1}(X, \mathbf{C}) \stackrel{\nu}{\longrightarrow} H^{1}\left(X, o_{X}\right)
$$

and so we must show $\operatorname{Im} \delta \cap H^{1}(X, \mathbf{R})=0$. Suppose $\delta(\varphi) \in H^{1}(X, \mathbf{R})$ and lift $\varphi$ to $\tilde{\varphi}$ on the desingularization $\tilde{X}$ of $X$. Then $\delta(\tilde{\varphi}) \in H^{1}(\tilde{X}, \mathbf{R})$ and so the imaginary part $\operatorname{Im} \tilde{\varphi}$ is a single-valued harmonic function on $\tilde{X}$ (determined on each component of $\tilde{X}$ up to an additive constant). Hence $\operatorname{Im} \tilde{\varphi}=0$, so therefore is $\tilde{\varphi}$ and also $\varphi$. Thus $\delta(\varphi)=0$ completing the proof modulo the verification concerning (6.3) which we now indicate briefly. Since this has nothing to do with the particular complex manifolds in question nor the dimension we shall give the argument for any conected compact complex manifold $P$ of dimension $n$ with $X$ a divisor (the dimension 2 in (6.3) being now replaced by $n$ throughout).

Let $\Phi^{n}$ denote the sheaf of closed $C^{\infty} n$-forms on $P-X, \Phi^{n}(X)$ its direct image sheaf on $P$

and we have a pairing

$$
\beta^{\prime}: H^{0}\left(P, \Phi^{n}(X)\right) \rightarrow H^{n}(P-X, \mathbf{C})
$$

$$
H_{\text {comp }}^{n}(P-X, \mathbf{C}) \otimes H^{0}\left(P, \Phi^{n}(X)\right) \rightarrow H_{\text {comp }}^{n}\left(P-X, \Phi^{n}\right) .
$$

Moreover, as in the proof of the $C^{\infty}$ de Rham theorem on $P-X$, we have isomorphisms

$$
\begin{aligned}
H_{\text {comp }}^{n}\left(P-X, \Phi^{n}\right) \cong H_{\text {comp }}^{n+1}\left(P-X, \Phi^{n-1}\right) \cong \ldots & \cong H_{\text {comp }}^{2 n}(P-X, \mathbf{C}) \\
& \cong \mathbf{C}
\end{aligned}
$$

and the pairing $(6.4)$ induces the pairing on the top row of $(6.3)$ via $\beta^{\prime}\left(^{(}\right)$The compatibility

(1) This assumes some basic facts about how cup products are defined using resolutions. 
of (6.3) (i.e. the adjointness of $\alpha, \beta$ ) will now follow from the compatibility of (6.4) and the bottom pairing of $(6.3)$. This in turn is proved by interposing the pairing

$$
H_{\text {comp }}^{n}(P-X, \mathbf{C}) \otimes H^{0}\left(P, \Omega^{n}(X)\right) \rightarrow H_{\text {comp }}^{n}\left(P-X, \Omega^{n}\right)
$$

and using the commutative diagram (see Serre (18 p. 23))

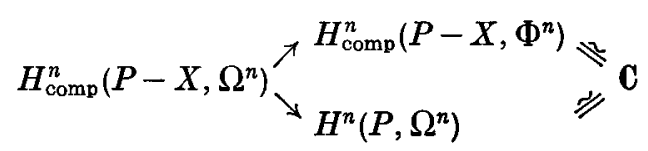

We come now to the proof of $(6.2)$ which follows the general lines of $\S 4$. First we must consider the local situation:

Lемма (6.5). Let $X$ be an analytic curve in $U=\left\{z \in \mathbf{C}^{2}|| z_{i} \mid<\varepsilon, i=1,2\right\}$ and let $f_{i}=0$ $(i=1, \ldots, r)$ be the equations of its branches through 0 . Then, for small $\varepsilon$, we have

(i) the 1 -forms $d f_{i} / f_{i}, i=1, \ldots, r$ give a basis of $H^{1}(U-X)$,

(ii) the 2-forms $d f_{i} d f_{r} / f_{i} f_{r}, i=1, \ldots, r-1$ give a basis of $H^{2}(U-X)$.

Proof. Put $X^{\prime}=X-\{0\}, U^{\prime}=U-\{0\}$, then $U-X=U^{\prime}-X^{\prime}$ and for small $\varepsilon, X^{\prime}$ consists of $r$ punctured dises. The exact cohomology sequence of the pair $\left(U^{\prime}, U^{\prime}-X^{\prime}\right)$ gives

$$
\begin{gathered}
H^{1}\left(U^{\prime}-X^{\prime}\right) \cong H^{2}\left(U^{\prime}, U^{\prime}-X^{\prime}\right) \cong H^{0}\left(X^{\prime}\right) \cong \mathbf{C}^{r} \\
0 \rightarrow H^{2}\left(U^{\prime}-X^{\prime}\right) \rightarrow H^{3}\left(U^{\prime}, U^{\prime}-X^{\prime}\right) \rightarrow H^{3}\left(U^{\prime}\right) \rightarrow 0 \\
\| 2
\end{gathered}
$$

This shows that $H^{1}(U-X)$ and $H^{2}(U-X)$ have dimensions $r, r-1$, respectively. Morever, the homomorphisms

$$
H^{i}\left(U^{\prime}-X^{\prime}\right) \rightarrow H^{i-1}\left(X^{\prime}\right) \quad i=1,2
$$

are given by taking residues (if we start from a meromorphic form with simple poles on $X^{\prime}$ ). From this it follows easily that the forms described in (i), (ii) give generators.

Passing now to the global situation let $X$ be an algebraic curve in $P_{2}$ with equation $f=0$ and consider the complex of sheaves

$$
0 \rightarrow \Omega^{0} \stackrel{d}{\longrightarrow} \Omega^{1}(X) \stackrel{d}{\longrightarrow} \Omega^{2}(2 X) \rightarrow 0
$$

where $\Omega^{1}(X)$ denotes meromorphic 1-forms with simple poles on $X, \Omega^{2}(2 X)$ denotes 
meromorphic 2-forms with double poles on $X$ (and $\Omega^{0}$ denotes holomorphic functions). Let $h^{i}$ denote the cohomology sheaves of this complex and let $\tilde{h}^{i}$ denote the sheaf $U \mapsto H^{i}(U-X ; \mathbf{C})$. Then we have

LeмMA (6.7). The natural map $h^{i} \rightarrow \tilde{h}^{i}$ is an isomorphism for $i=0,1$ and an epimorphism for $i=2$. Moreover, $h^{2}$ is concentrated at the singular points of $X$.

Proof. This is trivial for $i=0$. For $i=1$, using (6.5) (i), we have only to prove that the $d f_{i} / f_{i}$ generate $h^{1}$. To see this let $\omega$ be (locally) a closed 1-form with $f \omega$ holomorphic. By (6.5) (i), we can find constants $c_{i}$ so that $\omega-\Sigma_{i} c_{i} d f_{i} / f_{i}$ has no periods in $U^{\prime}-X^{\prime}$, hence is holomorphic in $U^{\prime}$, hence holomorphic in $U$ (Hartog's theorem), and therefore equal to $d \varphi$ for some holomorphic $\varphi$. This proves $\left(^{1}\right) h^{1} \cong \tilde{h}^{1}$. Next, $h^{2} \rightarrow \tilde{h}^{2}$ is epimorphic by (6.5) (ii) -since $f^{2} d f_{1} d f_{r} / f_{i} f_{r}$ is holomorphic. Finally at a non-singular point of $X$, taking local coordinates $(x, y)$ so that $X$ is $x=0$ we see that any local section of $\Omega(2 X)$ is of the form

$$
\omega=x^{-2} \varphi(y) d x d y+x^{-1} d x d \psi(y)+\theta(x, y) d x d y
$$

where $\varphi, \psi, \theta$ are holomorphic functions. Hence $\theta(x, y) d x d y=d \eta$ with $\eta$ a holomorphic 1-form and

$$
\omega=d\left(-x^{-1}(\varphi(y) d y+\psi(y) d x)+\eta\right)
$$

is in $d \Omega^{1}(X)$, proving that $h^{2}=0$ outside the singular points of $X$.

We now compare the complex (6.6) (denoted briefly by $\Omega$ ) with the complex of sheaves $\tilde{\Omega}$ : the direct image of the de Rham complex of $P_{2}-X$ under the inclusion $i: P_{2}-X \rightarrow P_{2}$. By the Bott vanishing theorems the cohomology groups $H^{q}(q \geqslant 1)$ of all sheaves in $\Omega$ vanish. Hence for both $\Omega$ and $\tilde{\Omega}$ the de Rham groups coincide with the hypercohomology. Hence we get two spectral sequences:

$$
\begin{aligned}
& E_{2}^{p, q}=H^{p}\left(P_{2}, h^{q}\right) \rightarrow D R^{*}(\Omega) \\
& \widetilde{E}_{2}^{p, q}=H^{p}\left(P_{2}, \tilde{h}^{a}\right) \rightarrow D R^{*}(\tilde{\Omega})=H^{2}\left(P_{2}-X\right) .
\end{aligned}
$$

and a homomorphism $E_{r} \rightarrow \widetilde{E}_{r}$. By Lemma (6.7) we see that $E_{2}^{p, q} \rightarrow \widetilde{E}_{2}^{p, q}$ is an isomorphism except for $(p, q)=(0,2)$ and there it is an epimorphism. From the spectral sequences we then see that

$$
E_{\infty}^{2,0} \cong \widetilde{E}_{\infty}^{2,0}, \quad E_{\infty}^{1,1} \cong \widetilde{E}_{\infty}^{1,1}
$$

and $E_{\infty}^{0,2} \rightarrow \widetilde{E}_{\infty}^{0,2}$ is an epimorphism.

(1) This argument (given in Atiyah-Hodge [4]) does not require us to be in dimension 2. 
This implies that $D R^{2}(\Omega) \rightarrow D R^{2}(\tilde{\Omega}) \cong H^{2}\left(P_{2}-X\right)$ is an epimorphism which completes the proof of $(6.2)$.

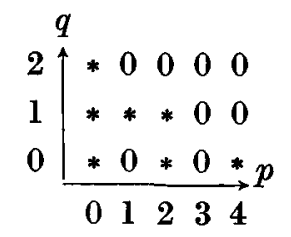

Diagram of $E_{2}^{p, q}$ with non-zero terms marked *.

\section{GHAPTER II. APPLICATIONS TO THE THEORY OF LACUNAS}

\section{§ 7. Introduction}

The results of Chapter I will now be applied to the theory of hyperbolic differential operators with constant coefficients. The notations and definitions will be those of Part I, Chapter 3, but we remind the reader briefly of the situation.

Linear differential operators with constant coefficients are written as polynomials $P(D)$ in the imaginary gradient $D=\partial / i \partial x$ with respect to $n$ variables $x$. We employ two $\mathrm{C}^{n}$-spaces $Z=Z_{n}=\{\xi, \ldots)$ and $Z^{\prime}=Z_{n}^{\prime}=\{x, \ldots\}$ with biorthogonal coordinates $\xi_{1}, \ldots$ and $x_{1}, \ldots$ and a duality $x \xi=x_{1} \xi_{1}+\ldots+x_{n} \xi_{n}$. Let $0 \neq \vartheta \in \operatorname{Re} Z$. We say that $P(D)$ is hyperbolic with respect to $\vartheta$ if $P(D)$ has a fundamental solution $E(P, x)=E(P, \vartheta, x)$ with support in a proper cone with its vertex at the orgin which, apart from its vertex is contained in the half-space $x \vartheta>0, x \in \operatorname{Re} Z^{\prime}$. Here $E$ is a distribution. The class of these operators or their characteristic polynomials is denoted by hyp $(\vartheta)$ and we write hyp $(\vartheta, m)$ for the set of $P \in$ hyp $(\vartheta)$ of degree $m$. The subsets of homogeneous elements in each class will be denoted by Hyp $(\vartheta)$ and Hyp $(\vartheta, m)$. The class hyp $(\vartheta)$ can be characterized algebraically: $P \in$ hyp $(\vartheta)$ if and only if $a(\vartheta) \neq 0$ and $P(\xi+\tau \vartheta) \neq 0$ for all real $\xi$ when $|\operatorname{Im} \tau|$ is big enough. Here $a(\xi)$ denotes the principal part of $P$. It is easy to see that $P \in$ hyp $(\vartheta)$ implies $a \in$ hyp $(\vartheta)$ and that $P$ and $a$ have the same lineality. The lineality $L(Q)$ of a polynomial $Q$ is the maximal linear subspace of $Z$ along which $Q$ is constant. In particular, if $a \in \operatorname{Hyp}(\vartheta)$ and the degree of $b$ is less than that of $a$, it is not true in general that $a+b \in$ hyp $(\vartheta)$. For this it is both necessary and sufficient that $b(\xi+i \vartheta) a(\xi+i \vartheta)^{-1}$ be bounded when $\xi$ is real (L. Svensson [20]).

When $a \in \operatorname{Hyp}(\vartheta, m)$, let $A$ be the complex hypersurface $a(\xi)=0, \xi \in Z$. The hyperbolicity then means that every straight line with the direction $\vartheta$ meets $\operatorname{Re} A$ in $m$ points. The component of $\operatorname{Re} Z-\operatorname{Re} A$ that contains $\vartheta$ is an open convex wedge $\Gamma=\Gamma(A, \vartheta)$ whose 
edge coincides with $\operatorname{Re} L(a)=\operatorname{Re} L(A)$. The dual cone $K=K(A, \vartheta)$ consists of all $x \in \operatorname{Re} Z$ such that $x \xi \geqslant 0$ for all $\xi \in \Gamma$. It is orthogonal to $\operatorname{Re} L(A)$. In particular, if $L(A)=0$, i.e. if $a(\xi)$ is complete in the sense that if depends on all variables, then $\Gamma$ is a proper cone and $K$ has a non-empty interior. When $P \in \operatorname{hyp}(\vartheta)$ and $a$ is the principal part of $P$, put $\Gamma(P, \vartheta)=\Gamma(A, \vartheta), K(P, \vartheta)=K(A, \vartheta)$. A main point of the theory is now that $P \in$ hyp $(\vartheta)$, $\eta \in \Gamma(P, \vartheta)$ implies $P \in$ hyp $(\eta)$ and that the fundamental solution $E(P)$ is given by the formula

$$
E(P, \vartheta, x)=(2 \pi)^{-n} \int e^{i x(\xi+i \eta)} P(\xi+i \eta)^{-1} d \xi
$$

where $\eta \epsilon-\Gamma(P, \vartheta)-c \vartheta$ with $c>0$ large enough. The integral, taken in the distribution sense, is then independent of the choice of $\eta$ and $K=K(P, \vartheta)$ is the convex hull of the support $S E$ of $E$. For this reason, $K$ is called the propagation cone of $P$. A simple argument shows that

$$
E(P, \vartheta, x)=E\left(P^{\prime}, \vartheta^{\prime}, x^{\prime}\right) \otimes \delta\left(x^{\prime \prime}\right)
$$

where $P^{\prime}$ and $\vartheta^{\prime}$ are the images of $P$ and $\vartheta$ in the quotient $Z / L(P), x^{\prime}$ are coordinates in its dual and the $x^{\prime \prime}$ are complementary coordinates in $\operatorname{Re} Z^{\prime}$. The dimension $n(P)$ of $Z / L(P)$, i.e. the codimension of $L(P)$ will be called the reduced dimension of $P$ and the polynomial $P^{\prime}$ and the fundamental solution $E\left(P^{\prime}\right)$ the reductions of $P$ and $E(P)$. Writing $P=a+b$ we also have the formula

$$
E(P, \vartheta, x)=\sum_{0}^{\infty}(-1)^{k} b(D)^{k} E\left(a^{k+1}, \vartheta, x\right) .
$$

The fundamental solution $E$ is holomorphic outside the wave front surfaçe $W(P, \vartheta)=$ $W(A, \vartheta)$, contained in $K(P, \vartheta)$ and defined as follows. The localization $P_{\xi}$ of $P$ at $\xi \in Z$ is a polynomial given by the formula

$$
t \rightarrow 0 \Rightarrow t^{m} P\left(t^{-1} \xi+\zeta\right)=t^{m_{\xi}} P_{\xi}(\zeta)+O\left(t^{m_{\xi}+1}\right) .
$$

and the requirement $P_{\xi}(\zeta) \neq 0$. The integer $m_{\xi}=m_{\xi}(P)$ is called the multiplicity of $\xi$ relative to $P$. It is also the degree of $P_{\xi}$. When $P \in$ hyp $(\vartheta)$ and $\xi \in \operatorname{Re} Z$ it turns out that $a_{\xi} \epsilon$ hyp $(\vartheta)$ is the principal part of $P_{\xi}$ and the wavefront surface $W(P, \vartheta)=W(A, \vartheta)$ is simply the union of all the local propagation cones $K_{\xi}(P, \vartheta)=K\left(P_{\xi}, \vartheta\right)$ when $0 \neq \xi \in \operatorname{Re} Z$. When $P$ is not complete, then $W(P, \vartheta)=K(P, \vartheta)$, otherwise $1+\operatorname{dim} W=\operatorname{dim} K$ and $W$ contains the boundary of $K$. Notice also the basic fact (I, Theorem 4.10') that

$$
0 \neq \xi \in \operatorname{Re} Z \Rightarrow \mathrm{S}\left(Q_{\xi}(D) E\left(P_{\xi}\right)\right) \subset \mathrm{SS}(Q(D) E(P))
$$

where $Q$ is any polynomial and S and SS denote "support of" and "singular support of" respectively. 
When $a \in \operatorname{Hyp}(\vartheta, m)$ and $x \in K(A, \vartheta)-W(A, \vartheta)$, the fundamental solution of a power $a^{k}$ of $a$ and its derivatives are given by the Herglotz-Petrovsky-Leray formulas (I, Theorem 7.16),

$$
\begin{aligned}
& D^{\nu} E\left(a^{k}, \vartheta, x\right)=\mathrm{const} \int_{\alpha^{*}}(x, \xi)^{q} \xi^{\nu} a(\xi)^{-k} \omega(\xi), q \geqslant 0, \\
& D^{\nu} E\left(a^{k}, \vartheta, x\right)=\mathrm{const} \int_{\gamma^{*}}(x, \xi)^{a} \xi^{\nu} a(\xi)^{-k} \omega(\xi), q<0 .
\end{aligned}
$$

Here const $\neq 0, q=m k-|\nu|-n$ is the homogeneity of the left side, $m$ being the degree of $a$, and

$$
\omega(\xi)=\Sigma(-1)^{k-1} \xi_{k} d \xi_{1} \ldots d \hat{\xi}_{k} \ldots d \xi_{n} .
$$

The integrands are closed rational $(n-1)$-forms on $(n-1)$-dimensional projective space $Z^{*}$ with poles on $A^{*}$ and $A^{*} \cup X^{*}$ respectively where $X$ is the complex hyperplane and a star denotes the image in projective space. Further $a^{*} \in H_{n-1}\left(Z^{*}-A^{*}, X^{*}\right)$ is a relative homology class with boundary $\beta^{*}=\beta(A, x, \vartheta)^{*}=\partial \alpha^{*} \in H_{n-2}\left(X^{*}-A^{*}, X^{*}\right)$ called the Petrovsky class and $\gamma^{*}=t_{x} \beta^{*} \in H_{n-1}\left(Z^{*}-A^{*} \cup X^{*}\right)$ where $t_{x}$ is induced by the tube operation from chains in $X^{*}$ to chains in $Z^{*}-X^{*}$ generated by the boundary of a small 2-disk in the normal bundle of $X^{*}$ as its center moves on $X^{*}$. A brief description of the relative class $\alpha^{*}$ runs as follows. Let $\mathbf{R}^{+}$be the positive reals and put $Z^{+}=\dot{Z}^{\prime} / \mathbf{R}^{+}$where $\dot{Z}=Z-\{0\}$. When $x$ is real, let $\alpha(x)^{+}$be the $(n-1)$-sphere $\operatorname{Re} Z^{+}$counted with multiplicity $\frac{1}{2}$ and oriented by $x \xi \omega(\xi)>0$. Then $\alpha^{*}=\alpha(A, \vartheta, x)^{*}$ is the homology class in $H_{n-1}\left(Z^{*}-A^{*}, X^{*}\right)$ of the image of $\alpha(x)^{+}$in $Z^{*}$ under any map

$$
\xi \rightarrow \xi-i v(\xi), \quad \xi \in \operatorname{Re} \dot{Z},
$$

where $v(\xi)$ is a real vector field in $C^{\infty}$ such that $v(\xi) \in \Gamma_{\xi}(\mathrm{A}, \vartheta)$ for all $\xi, x v(\xi)=0$ when $x \xi=0$ and $a(\xi+i t v(\xi)) \neq 0$ when $0<t \leqslant 1$. All such maps are homotopic. The details are given in Part I, section 6: Vector fields and cycles. We also remind the reader of the definitions of sharp fronts and lacunas (Part I, section 9). They will be used only in connection with fundamental solutions of hyperbolic operators but are, in fact, quite general. When $u$ is a distribution defined in an open subset $O$ of $\mathbf{R}^{n}$, let $\mathrm{S} u$ denote the support of $u$ and SS $u$ its singular support, i.e. the complement of the largest open subset of $O$ where $u$ is a $C^{\infty}$-function. When one of the coordinates is time so that $u$ is a wave, parts of $\mathrm{SS} u$ are called wave fronts. Let $L$ be a component of $O-\mathrm{SS} u$ and let $y \in \partial L$. We say that $u$ is sharp at $y$ from $L$ if $u$ has a $C^{\infty}$-extension from $L$ to $L \cap N$ for some neighbourhood $N$ of $y$. Of course, sharpness is an exception. In general, the wave or derivatives of it do not have limits at the front. When $u$ is sharp from $L$ at all points of $\partial L, L$ is said to be a 
lacuna for $u$. A lacuna $L$ is said to be strong if $u=0$ in $L$. Petrovsky [16], who seems to have introduced the word lacuna, only considers strong lacunas. The definition given here was suggested by L. Hörmander. Note that if $u$ has homogeneity $q$, i.e. if $u(\lambda x)=\lambda^{q} u(x)$ when $\lambda>0$, then $u$ is $\operatorname{sharp}$ from $L$ at the origin if and only if $u$ is a polynomial in $L$. In particular, $L$ is a strong lacuna for $u$ unless $q$ is an integer $\geqslant 0$.

When applying the definitions above to fundamental solutions $E=E(P, \vartheta, x)$ one is embarassed by the fact that SSE may be smaller than the wave front surface $W=W(P, \vartheta)$. It is therefore convenient to apply them also to components $L$ of the complement of $W$. Such components which are lacunas will be called regular lacunas. Every lacuna is then the union of regular lacunas and parts of $W$ not in SSE. The complement of the propagation cone $K(P, \vartheta)$ is a strong regular lacuna for $P$ called the trivial lacuna. If $P$ is not complete so that its reduced dimension $n(P)$ is less than $n$, the propagation cone $K(P, \vartheta)$ has no interior and the trivial lacuna is the only regular lacuna. But here, if $P^{\prime}$ is the reduction of $P,(7.2)$ shows that the study of $E$ reduces to that of $E^{\prime}=E\left(P^{\prime}, \vartheta^{\prime}, \cdot\right)$. In particular, the complement of the support of $E$ is the union of the trivial lacuna of $E$ and all strong lacunas of $E^{\prime}$. This union is of course connected.

Sharpness at the origin characterizes the lacunas of any $P \in$ hyp $(\vartheta)$ and $P$ and its principal part have, generally speaking, the same lacunas. The essential part of the exact statement (Lemma 9.6 of Part I) can be paraphrased as follows. Let $L$ be a component of $K(P, \vartheta)-W(P, \vartheta)$. Then all $E\left(P^{k}, \vartheta\right), .(k=1,2, \ldots)$, are sharp at the origin from $L$ if and only if $L$ is a lacuna for every $Q \in$ hyp $(\vartheta)$ whose principal part is a power of $a$.

Let $a \in \operatorname{Hyp}(\vartheta)$. When the Petrovsky condition

$$
\beta(A, \vartheta, x)^{*}=0 \text { in } H_{n-2}\left(X^{*}-X^{*} \cap A^{*}\right)
$$

holds for one $x$ in $K(A, \vartheta)-W(A, \vartheta)$, then, by virtue of $\left(7.5^{\prime}\right)$, all derivatives of $E\left(a^{k}, \vartheta, \cdot\right)$ of order $>m k-n$ vanish at $x$. Hence, if $L$ is the component of $K-W$ that contains $x, E\left(a^{k}, \vartheta, x\right)$ is a polynomial in $L$ and $L$ is a lacuna for all powers of a hence also for any $Q \in$ hyp $(\vartheta)$ whose principal part is a power of $a$. When $P \in$ hyp $(\vartheta)$ has principal part $a$, components $L$ of the complement of $W(A, \vartheta)$ for which the Petrovsky condition holds at all points are called Petrovsky lacunas.

Our main result on lacunas, announced as Theorem 10.9 of Part I, says that the fundamental solution $E\left(a^{k}, \vartheta\right)=E\left(a^{k}, \vartheta, x\right)$ of a high power $a \in \operatorname{Hyp}(\vartheta, m)$ has only Petrovsky lacunas, that its support is the propagation cone $K(A, \vartheta)$ and its singular support is the wave front surface $W(A, \vartheta)$. Moreover, this happens when $k$ exceeds a certain function of $m$ and $n$. The proof of this result is a simple consequence of the HerglotzPetrovsky-Leray formulas, the generalized Grothendieck theorem (see Proposition (5.2)) 
and the fact that the tube operation is injective and that the class $\alpha(A, x, \vartheta)^{*}$ is not zero when $x$ is inside the propagation cone (see $\S 8$ below). A more detailed version of our main result will be proved in $\S 9$. It has the following corollary of general interest. The proofs of (a) and (b) use Theorem 6.2, the proof of (c) Proposition 5.3. The properties (a) and (c) are true in all dimensions, (b) and (d) are more special.

THEOREM (7.7). Let $P \in$ hyp $(\vartheta, m)$ have principal part a and let $n(a)$ be the reduced dimension of $a$.

(a) If $n(a) \leqslant 3$ then

(i) all lacunas of $P$ are Petrovsky lacunas

(ii) $\mathrm{S} E(P, \vartheta)=K(A, \vartheta)$

(iii) $\operatorname{SS} E(P, \vartheta)=W(A, \vartheta)$.

(b) If $n(a)=4$ and $a$ is complete, (i) and (iii) hold.

(c) If $a$ is complete and $A^{*}$ has only normal crossings, (i) and (iii) hold.

(d) If $n\left(a_{\xi}\right) \leqslant 3$ for all $0 \neq \xi \in \mathbf{R}^{n}$, (iii) holds.

Note that (ii) says that $P$ has no non-trivial strong lacunas. Since the homogeneous wave operator in four variables has such a lacuna, (ii) does not hold in the cases (b) and (c). According to M. Mathisson [15], when $a$ is this wave operator and $P \neq a$, (ii) does in fact hold. It seems plausible that (ii) holds for non-homogeneous operators in general. On the other hand, K. G. Andersson (unpublished) has shown that the property $\operatorname{SS} E(P, \vartheta)=$ $\cup \mathrm{S} E\left(P_{\xi}\right)$ put forward as a conjecture in Part $\mathrm{I}$ is not true in general. There are homogeneous operators of order 4 in 6 variables for which the right side is not closed and inhomogeneous operators for which its closure is not equal to the left side.

\section{Lacunas of systems}

According to Lemma 3.2 of Part I, a differential operator $P=P(D)$ whose coefficients are square matrices is hyperbolic with respect to a real vector $\vartheta$ if and only if its determinant $\operatorname{det} P$ has that property. Further, if $Q(D)$ is the matrix of cofactors of $P(D)$, the formula

$$
E(P, \vartheta, x)=Q(D) E(\operatorname{det} P, \vartheta, x)
$$

connects the fundamental solutions of $P$ and $\operatorname{det} P$. It follows that the support or singular support of $E(P)$ is contained in the support or singular support of $E(\operatorname{det} P)$. Later (Theorem 14.20) we shall actually determine the singular support of $E(P)$ in a special case. 
The general case seems to be very complicated and we shall only prove the following result announced at the end of Part I.

THЕОВем (7.9). Let $P$ be a matrix-valued hyperbolic differential operator with determinant $\operatorname{det} P$ and let $E(P)$ and $E(\operatorname{det} P)$ be the corresponding fundamental solutions. Assume that the complex projective hypersurface given by the principal part of $\operatorname{det} P$ is non-singular. Then $E(P)$ and $E(\operatorname{det} P)$ have the same singular support and every lacuna for $P$ is a lacuna for $\operatorname{det} P$.

\section{\$ 8. Homology of hyperbolic hypersurfaces}

Lemma (8.1). Let $a \in \operatorname{Hyp}(\vartheta), 0 \neq x \in \mathbf{R}^{n}$, and let $A^{*}: a(\xi)=0, X^{*}: x \xi=0$ be the corresponding projective hypersurfaces. Then the tube operation

is injective.

$$
t_{x}: H_{n-2}\left(X^{*}-X^{*} \cap A^{*}\right) \rightarrow H_{n-1}\left(Z^{*}-A^{*} \cup X^{*}\right)
$$

Proof. The tube isomorphism $t_{x}$ is isomorphic, via Poincaré duality, to the coboundary

$$
\delta: H_{\mathrm{c}}^{n-2}\left(X^{*}-X^{*} \cap A^{*}\right) \rightarrow H_{c}^{n-1}\left(Z^{*}-A^{*}, X^{*}-X^{*} \cap A^{*}\right)
$$

where $H_{c}$ denotes cohomology with compact supports. Hence $\delta$ will certainly be injective if the preceding term $H_{c}^{n-2}\left(Z^{*}-A^{*}\right)$ in the exact sequence is zero. But this is true because $Z^{*}-A^{*}$ is a Stein manifold of complex dimension $n-1\left(H_{c}^{n-2}\right.$ being dual to $\left.H^{n}\right)$.

Lемма (8.2) with the notation of the preceding lemma assume further that $x \in W(A, \vartheta)$ but $x \in K(A, \vartheta)$. Then the class $\alpha(A, x, \vartheta)^{*} \in H_{n-1}\left(Z^{*}-A^{*}, X^{*}\right)$ does not vanish.

Proof. Let $\gamma^{*} \in H_{n-1}\left(Z^{*}-X^{*}, A^{*}\right)$ be the class of the relative cycle carried by $\Gamma(A, \vartheta)^{*}$. Clearly it will be sufficient to prove that $\alpha^{*} \cdot \gamma^{*}= \pm 1$. To compute this intersection number we shall choose a suitable representative $\alpha_{v}^{*}$ for $\alpha^{*}$ such that $v \in V(A, X, \vartheta)$ is given by

$$
v(\xi)=\operatorname{sgn}(x \xi)\left((x \vartheta)^{-1}(x \xi) \vartheta-\xi\right)
$$

for $\xi^{*} \in \Gamma(A, \vartheta)^{*}$. It is easy to check, from our assumptions on $x$, that (8.3) does indeed satisfy the conditions defining $V(A, X, \vartheta)$ (Part I $\S 6$ ) in the region $\Gamma(A, \vartheta)^{*}$ (the "inner oval"): the important point being that this inner oval does not meet the hyperplane $\operatorname{Re} X^{*}$ (so that $x \xi \neq 0$ there). The general construction for vector fields of $V(A, X, \vartheta)$ given in Part I, Lemma 6.7, then enables us to extend $v$ from $\Gamma(A, \vartheta)^{*}$ to the whole space. Now $\alpha_{v}^{*}$ is the sum of two cycles $\left({ }^{1}\right)$ arising from the two hemispheres $x \xi>0$ and $x \xi<0$ (on the

(1) Recall that in the definition of $\alpha_{v}$ the multiplicity of each hemisphere is $\frac{1}{2}$ and they are taken with opposite orientations. 
$(n-1)$-sphere $\left.Z^{+}\right)$by the map $\xi \rightarrow \xi-i v(\xi)$. It is clear that the images by (8.3) intersect $\Gamma(A, \vartheta)^{*}$ transversally at the point $\vartheta^{*}$. The only point is to show that the signs are the same and do not cancel. Now taking coordinates so that $\vartheta=(1,0, \ldots, 0), x=(1,0, \ldots, 0)$, $\xi^{\prime}=\left(\xi_{2}, \ldots, \xi_{n}\right)$ and using formula 6.18 of Part I it follows that the two cycles making up $\alpha_{v}^{*}$ are represented by $\xi^{\prime} \rightarrow \xi^{\prime}-i \xi^{\prime}$ (multiplicity $1 / 2$ ), $\xi^{\prime} \rightarrow \xi^{\prime}+i \xi^{\prime}$ (multiplicity $(-1)^{n-1} / 2$ ) This shows that the intersection numbers have the same sign.

Remark. Since the question of signs is crucial here the reader may get some reassurance from the following alternative argument valid for $n$ even and $A^{*}$ non-singular. In this case the vanishing of $\alpha^{*}$ is equivalent to the following: the boundary of the inner oval defines a homology class $\sigma$ on $A^{*}$ which is not a multiple of the class $\pi$ coming from intersections of hyperplanes. ( $\left.{ }^{(}\right)$Since $\sigma \cdot \pi=0$ (because $\operatorname{Re} X$ does not meet the inner oval) it is enough to show $\sigma^{2} \neq 0$. But $\sigma$ being one real component of an algebraic variety its normal bundle is isomorphic (by multiplication by $i$ ) to its tangent bundle. Hence the selfintersection is equal to its Euler characteristic which is 2 since $\sigma$ is a sphere of even dimension.

\section{§9. Lacunas}

We shall now give a detailed version of our main result. It uses two rather implicitly defined functions of which little is known except that they are finite. Let $b(\xi)$ denote complete real homogeneous polynomials in $n$ variables and let $k_{0}(m, n)$ and $k_{1}(m, n)$ be the least integer $k>0$ such that, respectively,

all forms $\xi^{\nu} b(\xi)^{-k} \omega(\xi)$ with $|\nu|=m k-n \geqslant 0$ and their conjugates span $H^{n-1}\left(Z^{*}-B^{*}\right)$

all forms $\xi^{\nu}(x \xi)^{a} b(\xi)^{-k} \omega(\xi)$ with $q=m k-n-|\nu|<0$ and their conjugates span $H^{n-1}\left(Z^{*}-B^{*} \cup X^{*}\right)$ for all $b$ and all real $x \neq 0$.

By proposition (5.2), both functions $k_{0}$ and $k_{1}$ are finite. In fact, they would be finite also if $b$ is not assumed to be real and the words "and their conjugates" deleted. Obviously, $k_{0}(m, 2)=k_{1}(m, 2)=1$ for all $m$. When $n=1$, we put $k_{0}=k_{1}=1$ by definition. Note that, also by definition, $m k_{0}(m, n)-n \geqslant 0$, but that there is no similar restriction on the function $k_{1}$. The function $k_{0}(m, n)$ cannot be used directly. Instead we shall employ the least majorant $k_{0}^{*}(m, n)$ of $k_{0}(m, n)$ which does not decrease as $m$ or $n$ increases. It is of course conceivable that $k_{0}^{*}=k_{0}$. In any case, the implication

$$
m \geqslant 3 \Rightarrow k_{0}^{*}(m, 3)=1
$$

is just another way of stating Theorem 6.1.

(1) This is the version given by Petrovsky.

12-732907 Acta mathematica 131. Imprimé le 11 Décembre 1973 
Lемм A (9.4). Let $a \in \operatorname{Hyp}(\vartheta, m)$ be complete and let $P^{(k)},(k=1,2, \ldots)$, be operators in hyp $(\vartheta, \mathrm{km})$ with principal parts $a^{k}$. Then

(i) $k \geqslant \min \left(k_{1}(m, n), k_{0}(m, m-1)\right) \Rightarrow$ all regular lacunas of $P^{(k)}$ are Petrovsky lacunas

(ii) $k \geqslant k_{0}^{*}(m, n) \Rightarrow \mathrm{S} E\left(P^{(k)}, \vartheta\right)=K(A, \vartheta)$.

Proof. By Lemma 9.6 of Part I, every regular (strong) lacuna of $P^{(k)}$ is also a regular (strong) lacuna of $a^{k}$. Hence it suffices to assume that the $P^{(k)}=a^{k}$ are homogeneous. Let $L$ be a component of $K(A, \vartheta)-W(A, \vartheta)$ and a lacuna for $a^{k}$. If $x \in L$ and $q=m k-n-|v|<0$, then by $\left(7.5^{\prime}\right)$

$$
0=\int_{t_{x} \beta^{*}}(x \xi)^{q} \xi^{\nu} a(\xi)^{-k} \omega(\xi)
$$

where $\beta^{*}=\beta(A, x, \vartheta)^{*} \in H_{n-2}\left(X^{*}-X^{*} \cap A^{*}\right)$ is the Petrovsky class. By (I.6.19), $\overline{t_{x} \beta^{*}}= \pm t_{x} \beta^{*}$ and hence the class $t_{x} \beta^{*}$ is orthogonal to all forms that appear in the integral and their conjugates. Hence, if $k \geqslant k_{1}(m, n)$, then by the definition of $k_{1}, t_{x} \beta^{*}=0$ so that, by Lemma 8.1, the Petrovsky condition holds in $L$. If $k \geqslant k_{0}^{*}(m, n-1)$ then $m k-n+1 \geqslant 0$ so that, if $|\nu|=m k-n+1$, then $q=-1$ and, taking one residue into $X^{*}$,

$$
0=\int_{\beta^{*}} \xi^{v} a(\xi)^{-k} \omega_{x}(\xi)
$$

where $\omega_{x}$ is defined in $X^{*}$ by $\omega(\xi)=d(x \xi) \wedge \omega_{x}(\xi)+O(x \xi)$. Now, by the definition of $k_{0}(m, n-1)$, all forms that appear in the integral and their conjugates $\operatorname{span} H^{n-2}\left(X^{*}-A^{*} \cap X^{*}\right)$ and hence, since $\overline{\beta^{*}}= \pm \beta^{*}$, the Petrovsky condition holds in $L$. This proves (i). To prove (ii), suppose that $L$ is a strong regular lacuna for $a^{k}$. Then it is also a lacuna and, since $k_{\mathbf{0}}^{*}(m, n) \geqslant k_{0}(m, n-1)$, (i) shows that the Petrovsky condition holds in $L$. Hence $\alpha^{*}$ can be lifted back to $H_{n-1}\left(Z^{*}-A^{*}\right)$. But then, if $x \in L$, putting $q=0$ in (7.5) we have

$$
0=\int_{\alpha^{*}} \xi^{\nu} a(\xi)^{-1} \omega(\xi), \quad|\nu|=m k-n
$$

Since $k \geqslant k_{0}(m, n)$, the definition of $k_{0}$ and the fact that $\overline{\alpha^{*}}= \pm \alpha^{*}$ shows that $\alpha^{*}=0$ in $H_{n-1}\left(Z^{*}-A^{*}\right)$ and hence also $\alpha^{*}=0$ in $H_{n-1}\left(Z^{*}-A^{*}, X^{*}\right)$. But this contradicts Lemma 8.2 so that (ii) follows.

We shall now prove a similar result when $a$ is not necessarily complete.

LEмм а (9.5). Let $a \in \mathrm{Hyp}(\vartheta, m)$ and let $P^{(k)},(k=1,2, \ldots)^{\prime}$ be operators in hyp $(\vartheta, \mathrm{km})$ with principal parts $a^{k}$. Let $n(a)$ be the reduced dimension of a. Then

$$
\begin{aligned}
\text { (i) } & k \geqslant k_{0}^{*}(m, n(a)) \Rightarrow \mathrm{S} E\left(P^{(k)}, \vartheta\right)=K(A, \vartheta) \\
\text { (ii) } & k \geqslant k_{0}^{*}(m-1, n(a)-1) \Rightarrow \operatorname{SSE}\left(P^{(k)}, \vartheta\right)=W(A, \vartheta)
\end{aligned}
$$


Proof. (ii) follows from (i). In fact, by the Localization theorem 4.10' of Part I,

$$
W(A, \vartheta) \supset \mathrm{SS} E\left(P^{k} \vartheta\right) \supset \underset{\xi \neq 0}{\bigcup} \mathrm{S} E\left(P_{\xi}^{(k)}, \vartheta\right) .
$$

Here $P_{\xi}^{(k)}$ has the principal part $a_{\xi}^{k}$ and $m\left(a_{\xi}\right)<m, n\left(a_{\xi}\right)<n(a)$ so that if the hypothesis of (ii) holds, then $\mathrm{S} E\left(P_{\xi}^{(k)}, \vartheta\right)=K\left(A_{\xi}, \vartheta\right)$ for all $\xi \neq 0$ and the conclusion of (ii) follows from the definition of $W(A, \vartheta)$. To prove (i), let $x=\left(x^{\prime}, x^{\prime \prime}\right)$ and $\xi=\left(\xi^{\prime}, \xi^{\prime \prime}\right)$ be corresponding divisions of coordinates such that $\xi^{\prime}=0$ characterizes $L(A) \neq 0$ and put $a^{\prime}\left(\xi^{\prime}\right)=a(\xi)$ and $P^{\prime(k)}\left(\xi^{\prime}\right)=P^{(k)}(\xi)$ so that $E\left(P^{(k)}\right)=E\left(P^{\prime(k)}\right) \otimes \delta\left(x^{\prime \prime}\right)$. Since the hypothesis of (i) implies the hypothesis of (ii) of Lemma 9.3 for $P^{\prime(k)}$ we have $\mathrm{S} E\left(P^{\prime(k)}\right)=K\left(A^{\prime}, \vartheta^{\prime}\right)$ and hence also $\mathbf{S} E\left(P^{(k)}\right)=K\left(A^{\prime}, \vartheta^{\prime}\right) \otimes\left\{0^{\prime \prime}\right\}=K(A, \vartheta)$.

The two preceeding lemmas constitute our main result on lacunas, support and singular support in detailed form. We can now produce a

Proof of Theorem 7.7. The items (ii) and (iii) of (a) follow if we combine (9.3) with Lemma 9.5. The item (i) is trivial unless $a$ is complete and in this case we can again use (9.3) and Lemma 9.4. To prove (b), use (9.3) and Lemma 9.5. The proof of (c) is not that immediate. The item (iii), however, follows from the Localization Theorem I.4.10',

$$
W(A . \vartheta) \supset \mathrm{SS} E(P, \vartheta) \supset \bigcup_{\xi \neq 0} \mathrm{~S} E\left(P_{\xi}, \vartheta\right) .
$$

Here, since $A^{*}$ is supposed to have only normal crossings, $a_{\xi}$ is just a product of linear factors so that $\mathrm{S} E\left(a_{\xi}, \vartheta\right)=K\left(A_{\xi}, \vartheta\right)$ and hence also $\mathrm{S} E\left(P_{\xi}, \vartheta=K\left(A_{\xi}, \vartheta\right)\right.$. Item (i) follows as in the proof of Lemma 9.3 from the fact (Proposition 5.3) that all forms that appear in (9.4) span $H^{n-1}\left(Z^{*}-A^{*} \cup X^{*}\right)$ so that the Petrovsky condition holds. Finally, (d) follows from item (ii) of (a) and the Localization Theorem I.4.10'.

Finally, we shall prove Theorem 7.9 about hyperbolic operators $P(D)$ whose coefficients are square matrices. Let $Q(D)$ be the associated matrix so that

$$
P(D) Q(D)=\operatorname{det} P(D) I
$$

where $I$ is a unit matrix. Let $a(D)$ be the principal part of $\operatorname{det} P(D)$. Since the complex projective hypersurface $A^{*}: a(\xi)=0$ is assumed to be non-singular, $a(\xi)=b(\xi)^{r}$ is a power of an irreducible polynomial $b(\xi)$ with the property that $\operatorname{grad} b(\xi) \neq 0$ when $\xi \neq 0$. If $Q$ has a factor which is a power of $b$, then by (9.6), $\operatorname{det} P$ has the same factor. Cancelling such factors we get

$$
P(D) Q_{0}(D)=p(D) I
$$

where $Q_{0}$ does not have the factor $b$ and the principal part of $P$ is a power of $b$, say $b^{s}$. According to this formula, the entries of $E(P)=Q_{0}(D) E(p) I$ have the form 


$$
F(x)=M(D) E(p, \vartheta, x)
$$

where $M$ is an entry of $Q_{0}$. Next we shall use the Localization Theorem 4.10' of Part I. Denoting localizations by an index $\xi$ we then have

$$
F_{\xi}=M_{\xi} E\left(p_{\xi}\right)
$$

and $\mathrm{SS} F$ contains the union of the $\mathrm{S} F_{\xi}$ for $0 \neq \xi$ real. Since the principal part of $p_{\xi}$ equals $b_{\xi}^{s}$ (Lemma 3.42 of Part I), and $b_{\xi}$ is constant or linear, the support of $E\left(p_{\xi}\right)$ is the entire propagation cone $K\left(p_{\xi}, \vartheta\right)$. This in turn is a a half-ray when $b(\xi)=0$, otherwise it is just the origin. Now, by assumption, there is an $M(\xi)$ which is not divisible by $b(\xi)$ and hence the real algebraic manifold $b(\xi)=0, M(\xi)=0$ has dimension $<n-1$. Except for this manifold, $M_{\xi}=M(\xi) \neq 0$ when $b(\xi)=0, \xi \neq 0$, and hence the union of the supports of the $F_{\xi}$ is dense in the wave front surface $W(\operatorname{det} P, \vartheta)=W(B, \vartheta)=\mathrm{W}(A, \vartheta)$. Hence $\operatorname{SS} E(P)$ contains $W(\operatorname{det} P, \vartheta$ ) which, by Theorem 7.7 equals $\operatorname{SS} E(\operatorname{det} P$ ). Since the opposite inclusion is trivial, the two singular supports are equal and the first part of the theorem is proved.

Next, write $M(D)=\Sigma M_{k}(D)$ as a sum of polynomials of homogeneity $k$. Assume that some component $L$ of $K(A, \vartheta)-W(A, \vartheta)$ belongs to a lacuna for $E(P, \vartheta, x)$. Then all the derivatives of $F(x)$ have limits as $x$ tends to zero along a ray in $L$. Precisely as in the proof of Lemma 9.6 of Part I this shows that, restricted to $L$, every $F_{k}=M_{k}(D) E\left(b^{s}, \vartheta, x\right)$ is a polynomial of homogeneity $m s-n-k, m$ being the degree of $b$ and, as before, $b^{s}$ the principal part of $p$. Now choose $N(D)=M_{k}(D)$ not divisible by $b$. Then, by $\left(7.5^{\prime}\right)$, high derivatives of $F_{k}$ are integrals over the Petrovsky tube $\gamma^{*}$ of rational differential forms with a multiple of $N(\xi)$ in the numerator and products $b(\xi)^{s}(x \xi)^{q},(q>0)$, in the denominator. Also, all multiples of $N(\xi)$ of sufficiently large homogeneity do occur. Making them contain $b(\xi)^{s-1}$ as a factor and applying Proposition 5.8 shows that $\gamma^{*}=0$. Since the tube operation is injective, $L$ is in fact a Petrovsky lacuna for $p(D)$ and hence also for $\operatorname{det} P(D)=$ $b(\xi)^{r-s} p(D)$. This finishes the proof.

\section{CHAPTER III.}

\section{SHARP FRONTS. THE LOCAL PETROVSKY CONDITION}

\section{§ 10. Introduction}

In this chapter we shall push the study of fundamental solutions of hyperbolic operators a bit further by examining their behaviour close to the wave front surface. We shall focus our interest on sharp fronts. To repeat the definition, let $a \in \mathrm{Hyp}_{\mathbf{p}}(\vartheta)$, let $E(a, \vartheta, x)$ be the corresponding fundamental solution, let $L$ be a component of the real complement 
of the wave front surface $W(A, \vartheta)$ and let $y \in \partial L$. Then $E$ is said to be $\left(C^{\infty}\right)$ sharp at $y$ from $L$ if $y$ has a neigbourhood $N$ such that the function $x \rightarrow E(a, \vartheta, x)$, holomorphic in $L$, has a $C^{\infty}$ extension from $L$ to $L \cap N$. When the function has a holomorphic extension to $L \cup N$ we say that $E$ is holomorphically sharp from $L$ (across $W(A, \vartheta)$ ) at $y$. Of course holomorphically sharp implies $C^{\infty}$ sharp. The two concepts are probably equivalent for fundamental solutions of hyperbolic operators.

Let $\beta(A, x, \vartheta)^{*}$ denote the Petrovsky class. As we have seen, the Petrovsky condition

$$
\beta(A, x, \vartheta)^{*}=0 \text { in } H_{n-2}\left(X^{*}-X^{*} \cap A^{*}\right)
$$

implies that $x$ belongs to a lacuna for all powers of $a$. There is a similar condition, the locaI Petrovsky condition, that implies that $E$ is holomorphically sharp from $L$ at $y$, namely

$$
\beta(A, x, \vartheta)^{*} \in H_{n-2}\left(Y^{*}-Y^{*} \cap A^{*}\right)
$$

when $x \in L$ is close enough to $y$. The formula should be taken in the sense that the left side belongs to the image of the right side induced by projections $Y^{*}-Y^{*} \cap A^{*} \rightarrow X^{*}-X^{*} \cap A^{*}$. A more precise statement is given at the end of this section together with a proof that (10.2) implies holomorphic sharpness from $L$ at $y$.

Example (10.3). When $n=2$, all Petrovsky classes vanish and all fronts are sharp. When $n=3$, all $X^{*}$ are just complex lines and, if $a \in \operatorname{Hyp}(\vartheta, m)$ is complete and $x$ belongs to a component $\delta$ of $K(A, \vartheta)-W(A, \vartheta)$, then $A^{*} \cap X^{*}$, which is invariant under complex conjugation, consists of $m$ possibly multiple points whose multiplicities are constant and the Petrovsky class $\beta(A, x, \vartheta)^{*}$ is represented by one-half times $\operatorname{Re} X^{*}$ detached from $\operatorname{Re} X^{*} \cap A^{*}$ (Part I, p. 167). As $x$ approaches $0 \neq y \in \partial L$ and $A$ does not contain the plane $\xi y=0$, at least two points of $A^{*} \cap X^{*}$ come together. The meaning of (10.2) is then that no such two points form a conjugate pair converging to a real point. When $A$ contains the plane $\xi y=0$, the right side of (10.2) is zero and the meaning of the formula is that the Petrovsky class vanishes. By the Herglotz-Petrovsky-Leray formulas (Part I, Theorem 7.16) ,the derivatives of order $m-2$ of the fundamental solution $E(a, \vartheta, x)$ are integrals over $\beta(A, x, \vartheta)^{*}$ of closed $(n-2)$-forms with poles only on $A^{*} \cap X^{*}$ which are rational functions of $x$ and the coefficients of $a$. The sufficiency of the local Petrovsky condition when $n=3$ is an immediate consequence of this statement. It is not difficult to show that in this case the condition is also necessary (even for $C^{\infty}$ sharpness) but this will not be done here.

In $\S$ I 2 we shall use Theorem I.9.3 to prove that the local Petrovsky condition is necessary when $y$ belongs to a regular piece of the wave front surface with a non- 
degenerate curvature, dual to a similar piece of $\operatorname{Re} A$. The necessity in the general case when $n>3$ is an open question.

The main point of this chapter is a study of sharpness at plane parts of the wave front surface, a case in a sense opposite to sharpness at curved pieces. The results are given in $\S 14$, the technical preparations in $\S 11$ and $\S 13$. The statements use the reduced wave front surface $\hat{W}(A, \vartheta)$ of a hyperbolic polynomial $a \in \operatorname{Hyp}(\vartheta, m)$ defind as the union of the local propagation cones $K\left(A_{\xi}, \vartheta\right)$ when $\xi$ is real and does not belong to the lineality $L(A)$ of $a$. The reduced wave front surface has codimension 1 in the propagation cone $K(A, \vartheta)$ and coincides with the wave front surface $W(A, \vartheta)$ when $a$ is a complete polynomial (see Lemma 1.5.17). Plane parts of $W(A, \vartheta)$ correspond to conical points of Re $A^{*}$, i.e. points $\eta^{*}$ such that the lineality $L\left(A_{\eta}\right)$ of the localized polynomial $a_{\eta}$ has minimal dimension, i.e. $L\left(A_{\eta}\right)=\mathrm{C} \eta$. The plane part in question is then simply the local propagation cone $K\left(A_{\eta}, \vartheta\right) \subset \operatorname{Re} Z^{\prime}$ spanning the hyperplane $x \eta=0$. We say that $y \in K\left(A_{\eta}, \vartheta\right)$ is a simple point of $W(A, \vartheta)$ if, close to $y, W(A, \vartheta)$ coincides with $K\left(A_{\eta}, \vartheta\right)$. Our main result (Theorem 14.1) is now that if $y \in K\left(A_{\eta}, \vartheta\right)-\hat{W}\left(A_{\eta}, \vartheta\right)$ is a simple point of $W(A, \vartheta)$ then, for all $k>0, E\left(a^{k}, \vartheta, \cdot\right)$ is holomorphically sharp at $y$ from both sides of $K\left(A_{\eta}, \vartheta\right)$. The proof is a somewhat lengthy verification that the local Petrovsky criterion holds. It employs certain results of a $§ 15$, Local hyperbolicity, which is interesting in itself and simplifies parts of section 2.5 of Part I. As a comparatively simple corollary (Theorem 14.18) we can compute the jump $J\left(a^{k}, \vartheta, x\right)$ of $E\left(a^{k}, \vartheta, \cdot\right)$ at $y$, defined as the equivalence class of $E$ modulo functions holomorphic close to $y$. The formula is simply

$$
J\left(a^{k}, \vartheta, x\right)=\theta(x \eta)(x \eta)^{k(m-p)-1} H(x)
$$

where $\theta$ is the Heaviside function, $p=m\left(a_{\eta}\right)$ the degree of $a_{\eta}$ and $H(x)$ is a holomorphic function of homogeneity $k p+1-n$. In the special case when $y$ is in a lacuna for all powers of $a_{\eta}$, considered as a polynomial on $Z / \mathrm{C}_{\eta}$, i.e. when the Petrovsky condition in dimension $n-1$ holds,

$$
\beta\left(A_{\eta}, y, \vartheta\right)^{*}=0 \quad \text { in } H_{n-3}\left(Y^{*}-Y^{*} \cap A_{\eta}\right)
$$

then $H(x)$ is a polynomial and hence has to vanish when $k p<n-1$. In this case, then, $E\left(a^{k}, \vartheta, \cdot\right)$ is holomorphic across $W(A, \vartheta)$ at $y$.

We give an application of this to symmetric hyperbolic systems in three variables (Theorem 14.20).

Note that the condition (10.4) together with $k p<n-1$ implies that $E_{\eta}\left(a^{k}, \vartheta, x\right)=$ $E\left(a_{\eta}^{k}, \vartheta, x\right)$ vanishes close to $y$. Hence our result indicates that the inclusion (Part I, p. 145) 


$$
\mathrm{SS} E \supset \bigcup_{\eta \neq 0} \mathrm{~S} E_{\eta}
$$

may be an equality. This, however, is not true. As pointed out in the introduction, examples by K. G. Andersson show that the inclusion may be strict.

We end this section by stating a precise form of (10.2).

The local Petroviky condition (10.5).

Let $a \in \mathrm{Hyp}(\vartheta)$ be complete, let $L$ be a component of the real complement of $W(A, \vartheta)$ and let $\mathbf{0} \neq y \in \partial L$. Let $\pi_{x}$ be diffeomorphisms $Y \rightarrow X$ continuous in $x$ close to $y$ such that $\pi_{y}$ is the identity and let $l_{y}$ be a smooth path in $L$ ending at $y$. Suppose that, for some choice of $l_{y}$ and the family $\pi_{x}$ we have

$$
x \in l_{y} \Rightarrow \beta(A, x, \vartheta)^{*} \ni \pi_{x} \beta^{*}
$$

where $\beta^{*}$ is a $(n-2)$-cycle in $Y^{*}$ avoiding $A^{*}$. Then $E(a, \vartheta, x)$ is holomorphically sharp at $y$ from $L$.

Proof. It suffices to show that $l_{y}$ has a neighbourhood $L_{y}$ in $L$ such that when $x \in L_{y}$ is close to $y$, the class $t_{x} \beta(A, \vartheta, x)^{*} \in H_{n-1}\left(Z^{*}-A^{*} \cup X^{*}\right)$ contains a cycle $\gamma^{*}$ which does not depend on $x$. In fact, in this case the Herglotz-Petrovsky-Leray formulas show that the derivatives of $E(a, \vartheta, x)$ of order $>m-n$ have holomorphic extensions from $L_{y}$ to a complex neighbourhood of $y$. Now, by assumption, there is a $(n-2)$-cycle $\beta_{y}^{*} \subset Y^{*}-Y^{*} \cap A^{*}$ such that $\pi_{x} \beta_{y}^{*}$ is in the Petrovsky class $\beta(A, x, \vartheta)^{*}$ for all $x \in l_{y}$. It follows from Lemma I.6.23 that if $x^{\prime}$ is sufficiently close to such an $x$, then $\pi_{x^{\prime}} \beta_{y}^{*}=\pi_{x^{\prime}} \pi_{x}^{-1} \pi_{x} \beta_{y}^{*}$ is in the Petrovsky class $\beta\left(A, x^{\prime}, \vartheta\right)^{*}$. Hence the previous statement holds for all $x$ in a certain neighbourhood $L_{y}$ of $l_{y}$. Let $\gamma^{*}=t_{y} \beta_{y}^{*} \subset Z^{*}-A^{*} \cup Y^{*}$ be a small tube around $\beta_{y}^{*}$. When $x$ is very close to $y$, this is also a tube in $Z^{*}-A^{*} \cup X^{*}$ around $\pi_{x} \beta_{y}^{*}$ and hence $\gamma^{*} \in t_{x} \beta(A, x, \vartheta)^{*}$ provided, in addition, $x \in L_{y}$. This completes the proof.

\section{§ 11. Representatives of the Petrovsky class}

Let $a \in \mathrm{Hyp}(\vartheta)$ and $x \in W(A, \vartheta)$. According to the general theory, representatives of the Petrovsky homology class $\beta(x)^{*}=\beta(A, x, \vartheta)^{*} \in H_{n-2}\left(X^{*}-X^{*} \cap A^{*}\right)$ may be obtained as follows. Let $V(A, X, \vartheta)$ be all real $C^{\infty}$-vector fields

\section{$\operatorname{Re} \dot{X} \ni \xi \rightarrow v(\xi) \in \operatorname{Re} X$}

which are homogeneous of degree $1, \lambda>0 \Rightarrow v(\lambda \xi)=\lambda v(\xi)$ and are such that

$$
v(\xi) \in \Gamma\left(A_{\xi}, \vartheta\right) \cap \operatorname{Re} X
$$


for all $\xi$. Then $\beta(x)^{*}$ is the homology class in $H_{n-2}\left(X^{*}-X^{*} \cap A^{*}\right)$ of the image in projective space of the cycles

$$
\operatorname{Re} \dot{X} \ni \xi \rightarrow \xi-i t v(\xi)
$$

with $t>0$ sufficiently small, oriented by $\omega_{x}(\xi)>0$. Here $\omega_{x}(\xi)$ is a $(n-2)$-form on $X$ defined by $\omega(\xi)=d(x \xi) \wedge \omega_{x}(\xi)+O(x \xi)$. The map or cycle (11.1) is said to represent the class $\beta(x)^{*}$. We shall now construct other maps with the same property.

LemmA (11.2). Let $S \subset \mathbf{R}^{n-2}$ be a $(n-2)$-sphere and a $C^{\infty}$ manifold and let

$$
\xi: S \rightarrow \xi(S) \subset \operatorname{Re} X
$$

be a diffeomorphism and assume that every half-ray through the origin in $\operatorname{Re} X$ meets $\xi(S)$ precisely once. Let $I$ be an interval around the origin in $\mathbf{R}$ and let

$$
\zeta=\zeta(t): S \times I \rightarrow X
$$

be $a C^{\infty}$ map such that $\zeta(0)=\xi$ and

$$
D \zeta(0) \in \Gamma\left(A_{\xi}, \vartheta\right) \cap X, \quad D=-\partial / i \partial t,
$$

for all $\xi$ and let $\gamma(t)$ be the cycle

$$
\zeta(t): S \rightarrow \dot{X}
$$

oriented by $\omega_{x}(\xi)>0$. Then $\gamma(t)^{*} \in \beta(A, x, \vartheta)^{*}$ provided $t>0$ is small enough.

Proof. When $\zeta(t)=\xi-i t v(\xi), v(\xi)=D \zeta(0)$, is linear in $t$, this is just the definition of $\beta^{*}$. Further, it follows from Lemma I.5.9 (the inner continuity of the local $\Gamma$-cones) and a covering argument that

$$
a(\xi+\varphi(\xi)-i t(v(\xi)+\psi(\xi))) \neq 0
$$

for all real $\xi$, all sufficiently small real functions $\varphi$ and $\psi$ of homogeneity 1 and all small $t \neq 0$. Hence, if $t>0$ is small enough, the maps $\zeta(t)$ and $\xi-i t v(\xi)$ are homotopic in $Z-A$ and the lemma follows. Note also that if $U$ is an open subset of $S$ and $\zeta_{0}: U \times I \rightarrow X$ has the desired property (11.3), then there is a $\zeta: S \times I \rightarrow \dot{X}$ satisfying (11.3) everywhere and coinciding with $\zeta_{0}$ on any given compact subset of $U$ when $t$ is small. The proof goes by a partition of unity and the interior continuity of the local cones.

We shall now use the preceding lemma to specify a situation where the generalized Petrovsky condition holds. We shall encounter it both in $\S 12$ and $\S 14$.

A point $y \in W(A, \vartheta)$ is said to be simple if $y \in K\left(A_{\xi}, \vartheta\right)$, i.e. $\Gamma\left(A_{\xi}, \vartheta\right) \cap \operatorname{Re} Y=\varnothing$, implies that $\xi$ is a multiple of precisely one $\eta \in \operatorname{Re} A$. In case there is a finite number of such rays $R_{\eta}, y$ is said to be quasi-simple. We shall only consider simple points but make 


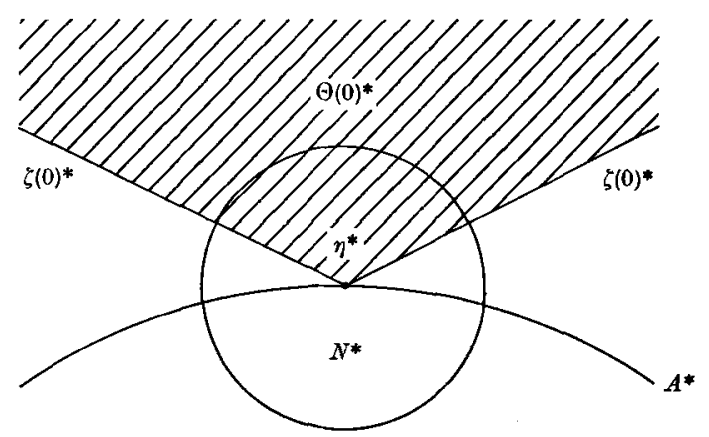

Fig. 1.

occasional remarks about quasi-simple ones. Now, let $L$ be a component of the complement of the wave front surface and let $y \in \partial L$ be a simple point. Let $l_{y}$ be a smooth path in $L$ ending at $y$ and, for $x$ close to $y$ define a projection $\pi_{x}: Z \rightarrow X$ by $\pi_{x} \xi=\xi-(x, \xi)(x, \lambda)^{-1} \lambda$ where $\lambda \in \operatorname{Re} Z$ is not in $Y$. We are going to define a $(n-2)$-cycle $\gamma^{*}$ in $Y^{*}-Y^{*} \cap A^{*}$ such that $\pi_{x} \gamma^{*}$ represents the Petrovsky class $\beta(A, x, \vartheta)^{*}$ when $x \in l_{y}$ is close to $y$. This can only be done under certain conditions and $\gamma^{*}$ is obtained by deflecting a cycle $\gamma_{0}^{*}$ in $\left(Y^{*}-Y^{*} \cap A^{*}\right) \cup \eta^{*}$ from the point $\eta^{*} \in A^{*}$. Our conditions are now as follows.

There are orientable manifolds $\zeta(t), \theta(t) \subset Y$ of dimensions $n-2$ and $n-1, C^{\infty}$ functions of $t$ when $t \geqslant 0$ and $t>0$ respectively such that

(i) $\zeta(0) \subset \operatorname{Re} Y$ is transversal to rays through the origin and contains $\pm \eta$.

(ii) There is a projective neighbourhood $M^{*}$ of $\eta^{*}$ such that, putting $D=-d / i d t$ and letting $\xi$ and $\chi$ be corresponding points of $\zeta(0)$ and $D \zeta(0)$,

$$
x \in l_{y} \text { or } x=y, \xi^{*} \in M^{*} \Rightarrow \pi_{x} \chi \in \Gamma\left(A_{\pi_{x} \xi}, \vartheta\right)
$$

when $x$ is close enough to $y$, except when $x=y, \xi^{*}=\eta^{*}$.

(iii) There is a projective neighbourhood $N^{*}$ of $\eta^{*}$ such that

$$
\zeta(t)^{*} \cap A^{*} \cap N^{*}=\eta^{*}, \pi_{x} \zeta(t)^{*} \cap A^{*} \cap N^{*}=\varnothing
$$

for all $x \in l_{y}$ close enough to $y$ and all small enough $t>0$. Also,

$$
\partial \theta(t)^{*} \cap N^{*}=\zeta(t)^{*} \cap N^{*}
$$

when $t>0$ is small enough.

(iv) For every small enough $t>0$ there is a projective neighbourhood $N(t)^{*} \subset V^{*}$ of $\eta^{*}$ such that

$$
\theta(t)^{*} \cap A^{*} \cap N(t)^{*}=\eta^{*}, \quad \pi_{x} \theta(t)^{*} \cap A^{*} \cap N(t)^{*}=\varnothing
$$

when $x \in l_{y}$ is close enough to $y$. 
The schematic figure 1 illustrates the relative positions of $\theta(0)^{*}, \zeta(0)^{*}, A^{*}, \eta^{*}, N^{*}$.

Lемм А (11.4). Under these hypotheses, $E(a, \vartheta, \cdot)$ is holomorphically sharp at y from $L$.

Proof. Arguing as at the end of the proof of the preceding lemma, we may suppose that $\zeta(0)$ is a sphere $S$ around the origin in $\operatorname{Re} Y$ and that (ii) holds for all $\xi \in S$. By the preceding lemma we then know that to every $x \in l_{y}$ close enough to $y$, there is a $t(x)>0$ such that $\pi_{x} \zeta(t)^{*}$, oriented by $\omega_{y}(\xi)>0$, represents the Petrovsky class $\beta(A, x, \vartheta)^{*}$ when $0<t \leqslant t(x)$. Also, given any neighbourhood $M^{*}$ of $\eta^{*}$, we can assert that

$$
\pi_{x} \zeta(t)^{*} \cap\left(Z^{*}-M^{*}\right) \cap A^{*}=\varnothing
$$

when $x$ is close enough to $y$ and $t>0$ is small enough. Combining this with (iii) it follows that $\pi_{x} \zeta(t)^{*}$ represents $\beta(A, x, \vartheta)^{*}$ for all $x \in l_{y}$ close enough to $y$ and all sufficiently small $t>0$. We now fix a $t>0$ and a neighbourhood $N^{*}(t)$ of $\eta^{*}$ according to (iv), chosen so that $\partial N^{*}(t), \theta(t)^{*}, \partial \theta(t)^{*}$ are in general position. Dropping the argument $t$ we then put $\varphi^{*}=\theta^{*} \cap N^{*}$. Then $\partial \varphi^{*}=-\zeta^{*} \cap N^{*}+\theta^{*} \cap \partial N^{*}$ so that

$$
\gamma^{*}=\zeta^{*}+\partial \varphi^{*}=\left(\zeta^{*}-\zeta^{*} \cap N^{*}\right)+\theta^{*} \cap \partial N^{*}
$$

does not meet $A^{*}$. Also, by (iii) and (iv), $\pi_{x} \varphi^{*} \cap A^{*}=\varnothing$ when $x \in l_{y}$ is close enough to $y$. Hence, under the same hypothesis, $\pi_{x} \gamma^{*}$ belongs to the Petrovsky class $\beta(A, x, \vartheta)^{*}$. Since the class of $\gamma^{*}$ belongs to $H_{n-2}\left(Y^{*}-Y^{*} \cap A^{*}\right)$, this shows that the local Petrovsky criterion holds so that $E$ is holomorphically sharp at $y$ from $L$.

Note. When $y \in W(A, \vartheta)$ is quasi-simple, Lemma 11.4 holds provided the conditions (i) to (iv) hold at every $\eta^{*} \in A^{*}$ such that $y \in K\left(A_{\eta}, \vartheta\right)$. The proof is immediate and reflects the fact that when $x$ is close to $y, E(a, \vartheta, \cdot)$ can be written as the sum of a holomorphic function and integrals extended over neighbourhoods of the points $\eta^{*}$.

\section{\$12. Sharpness at points of the wave front surface with non-degenerate curvature}

Let $F$ be a conical regular hyper-surface in $\operatorname{Re} Z^{\prime}=\mathbf{R}^{n}$, let $y \in F$ and let $f(x)=0$ with $f_{x}=\operatorname{grad} f \neq 0$ at $y$ be the equation of $F$ close to $y$. Let $f_{x x}$ denote the matrix $\left(\partial^{2} f / \partial x_{j} \partial x_{k}\right)$ and $f_{x x}[z]$ the corresponding quadratic form $\Sigma\left(\partial^{2} f / \partial x_{j} \partial x_{k}\right) z_{j} z_{k}$ and put $f_{x} z=\Sigma\left(\partial f / \partial x_{j}\right) z_{j}$. The curvature form of $F$ at $x$ is then the form $f_{x x}[z]$ restricted to the tangent plane $f_{x} z=0$. It depends on the sign of $f$. We assume that it is not degenerate. Taking $f$ to be homogeneous of degree $m \neq 1$, an equivalent condition is that $f_{x x}$ be nondegenerate. Then the map $x \rightarrow \xi=f_{x}$ is invertible close to $y$ and maps $F$ locally onto its dual surface $F^{*}$ defined by $f^{*}=0$ where $f^{*}(\xi)=f(x), \xi$ close to $\eta=f_{x}(y)$. It is easy to see that $F^{*}$ is independent of the choice of $f$ and $m$ and for convenience we choose $m=2$ in the sequel. Then $f^{*}$ has homogeneity 2 also. We shall see that the maps $x \rightarrow \xi=f_{x}$ and 
$\xi \rightarrow x=f_{\xi}^{*}$, the matrices $f_{x x}$ and $f_{\xi \xi}^{*}$ and the curvature forms of $F$ and $F^{*}$ at corresponding points are inverses of each other. In fact, $\xi=f_{x}$ gives $d \xi=f_{x x} d x$, i.e. $\partial / \partial \xi=f_{x x}^{-1} \partial / \partial_{x}$ and hence $f_{\xi}^{*}=f_{x x}^{-1}(\partial / \partial x) f(x)=f_{x x}^{-1} \xi=f_{x x}^{-1} f_{x x} x=x$. Also, $f_{\xi \xi}^{*}=\partial f_{\xi}^{*} / \partial \xi=\partial x / \partial \xi=f_{x x}^{-1} \partial x / \partial x=f_{x x}^{-1}$. Finally, putting $u=f_{x x} z$ we have $f_{x x}[z]=f_{\xi \xi}[u]$ and $f_{x} z=\xi z=\xi f_{x x}^{-1} u=\xi f_{\xi \xi} u=f_{\xi}^{*} u$.

The present situation applies when $F^{*}$ is a neighbourhood of $\eta$ in $\operatorname{Re} A$ and $y$ is simple point of $W(A, \vartheta)$ so that $F$ is a full neighbourhood of $y$ in $W(A, \vartheta)$. By Theorem I.9.3, whose assumptions should include also the nature of $F^{*}$, the fundamental solution $E(a, \vartheta)$ is $\operatorname{sharp}$ at $y$ from the side $\operatorname{sgn} f=$ const if and only if $(\operatorname{sgn} f)^{n}=(-1)^{\nu}$ where $v$ is the negative signature of the curvature form. We shall now see that this is equivalent to the local Petrovsky condition.

ThEOREM (12.1). Let $a \in \operatorname{Hyp}(\vartheta)$ and let $y \in W(A, \vartheta)$ be a simple point such that the corresponding piece of $\operatorname{Re} A$ is regular with a non-degenerate curvature. Then, if $E(a, \vartheta)$ is $C^{\infty}$-sharp at $y$ from one side of $W$, the local Petrovsky condition holds there.

Note (12.2). Theorem I.9.3 is proved in the real domain simply by using the method of stationary phase to compute an asymptotic expansion of $x \rightarrow E(a, \vartheta, x)$ as $x$ approaches $y$. The theorem above is a homological counterpart. It is intimately connected with the classical Picard-Lefschetz theorem according to which one turn in $Z$ around the complexification of $W$ induces an isomorphism of $H_{n-2}\left(X^{*}-X^{*} \cap A^{*}\right)$ that takes $\beta^{*}$ into $\beta^{*} \pm\left(\beta^{*}, \varepsilon^{*}\right) \varepsilon^{*}$ where $\varepsilon^{*}=\varepsilon(x)^{*}$ is a so-called vanishing class and the parenthesis denotes intersection number. The class $\varepsilon^{*}$ is a tube around $\partial e(x)^{*}$ where $e(x)^{*} \in H_{n-2}\left(X^{*}, A^{*} \cap X^{*}\right)$ is another vanishing class introduced at the end of the proof. The condition that $\beta^{*}$ be invariant, i.e. that $\left(\beta(x)^{*}, \varepsilon(x)^{*}\right)=0$ is in the present situation equivalent to the local Petrovsky condition.

Note (12.3). Theorem (12.1) shows that for non-degenerate points we have a topological criterion for sharpness. If $A^{*}$ is non-singular then this accounts for most points (i.e. for a dense open set of the wave-front set). Moreover we can also characterize sharpness at the exceptional points by the criterion that $\alpha_{x}^{*}$ should be invariant under the local fundamental group $\pi_{1}$ (of the complement of the dual of $A^{*}$ in complex projective space). The argument runs briefly as follows. Sharpness $\Rightarrow$ invariance is a consequence of the Herglotz-Petrowsky formula together with the Grothendieck theorem. For the opposite implication we use the Picard-Lefschetz theory as above to extend over all non-degenerate points and then extend over the remainder (having complex codimensions $\geqslant 2$ ) by the Hartogs extension theorem for several complex variables. Note that the local Petrowsky condition implies invariance under $\pi_{1}$, but the converse is a difficult unsolved 
problem in general. For singular $A^{*}$ the wave-front set $W$ contains many regions not covered by our discussion of sharpness so far-notably $W$ may contain flat pieces (open sets in hyperplanes)-and our main aim will be to get information on these.

Proof. We can choose linear coordinates and the equation $f^{*}=0$ of $\operatorname{Re} A$ at $\eta$ such that $\eta=(1,0, \ldots, 0), y=(0,1,0, \ldots, 0)$ and when $\xi_{2} / \xi_{1}, \ldots$ are small

$$
f^{*}(\xi)=2 \xi_{1} \xi_{2}+Q\left(\xi_{3}, \ldots, \xi_{n}\right)+\xi_{1}^{2} H^{*}\left(\xi_{3} / \xi_{1}, \ldots\right)
$$

Here $Q=-\xi_{3}^{2}-\ldots-\xi_{p}^{2}+\xi_{p+1}^{2}+\ldots, p=v+2$, is a non-singular quadratic form of signature $\nu$ and $H^{*}$ vanishes of order 3 at the origin. Hence

$$
f(x)=2 x_{1} x_{2}+Q^{-1}\left(x_{3}, \ldots, x_{n}\right)+x_{2}^{2} H\left(x_{3} / x_{2}, \ldots\right)
$$

where now $x_{1} / x_{2}, x_{3} / x_{2}, \ldots$ are small and $H$ vanishes of order 3 at the origin. We are going to verify the hypotheses of Lemma 11.4. First, let $l_{y}=l_{y}^{e}$ consist of all $x=\left(x_{1}, 1,0, \ldots\right)$ with $x_{1}+0$ small and given $\varepsilon=\operatorname{sgn} x_{1}$. Since $\operatorname{sgn} f(x)=\varepsilon$ for these $x$, they lie in a component of the real complement of $W(A, \vartheta)$ which we denote by $L^{\varepsilon}$. By Theorem I.9.3, $E(a, \vartheta)$ is sharp at $y$ from $L^{\varepsilon}$ if and only if $\varepsilon^{n}=(-1)^{p}$. Changing if necessary $f$ to $-f$ it suffices to consider the case $\varepsilon=1$. The projection $\pi_{x}$ on $X$ will be given by $\pi_{x} \xi=$ $\xi-(x, \xi)(y, \lambda)^{-1} \lambda$ where $\lambda=(0,1,0, \ldots)$. Hence

$$
x \in l_{y} \Rightarrow \pi_{x} \xi=\left(\xi_{1},-x_{1} \xi_{1}, \xi_{3}, \ldots\right) .
$$

We shall first define the chain $\theta(t), t>0$ small. It is given by the map

$$
\tau, u, s \rightarrow \theta(t)=\left(\tau s+i t, 0,(\tau s+i t) \tau u_{3}, \ldots, u_{p+1}, \ldots\right)
$$

where $\tau= \pm 1,0 \leqslant s \leqslant 1$ and $u_{3}, \ldots$ are real. The orientation induced by $\tau d u d s>0$, changes with $\tau$. The boundary $\partial \theta(t)$ has two parts corresponding to $s=1,0$, namely

$$
\zeta(t)=\theta(t, 1, \tau, u)=\left(\tau+i t, 0,(\tau+i t) \tau u_{3}, \ldots, u_{p+1}, \ldots\right)
$$

oriented by $d \tau d u>0$ and

$$
\zeta_{0}(t)=\left(i t, 0, i t \tau u_{3}, \ldots, u_{p+1}, \ldots\right)
$$

oriented by $d \tau d u<0$. Changing the signs of $\tau, u_{3}, \ldots, u_{p}$ does not change the range of $\zeta_{0}(t)$ but changes the orientation by a factor of $(-1)^{p-1}$. Hence $\zeta_{0}(t)$ vanishes if and only if $p$ is even, i.e. if and only if $E$ is sharp at $y$ from $L=L^{1}$. In the sequel we consider this case. Put 


$$
\varrho_{1}^{2}=u_{3}^{2}+\ldots+u_{p}^{2}, \quad \varrho_{2}^{2}=u_{p+1}^{2}+\ldots+u_{n}^{2}
$$

and measure the distance in projective space $Z^{*}$ of $\theta^{*}=\theta(t)^{*}=\theta(t, s, \tau u)^{*}$ from $\eta^{*}$ by $\varrho\left(\theta^{*}\right)$ where

$$
\varrho\left(\theta^{*}\right)^{2}=\varrho_{1}^{2}+\varrho_{2}^{2}|\tau s+i t|^{-2}
$$

Assuming $\varrho\left(\theta^{*}\right)$ small we then have

$$
f^{*}\left(\pi_{x} \theta\right)=\left(t^{2}-s^{2}\right)\left(x_{1}^{2}+\varrho_{1}^{2}\right)-2 i t \tau s\left(x_{1}+\varrho_{1}^{2}\right)+O(1)|s+i t|^{2} \varrho\left(\theta^{*}\right)^{3}
$$

We shall now verify the conditions (i) to (iv) of Lemma (11.4), $\zeta(t)$ being defined by (12.5). The condition (i) is then obvious. To verify (ii) note that

$$
-D \zeta(0)=\left(1,0, \tau u_{3}, \ldots, \tau u_{p}, 0, \ldots, 0\right)
$$

is homogeneous of degree 0 in

$$
\zeta(0)=\left(\tau, 0, u_{3}, \ldots, u_{n}\right)
$$

and that, close to $\pm \eta, \Gamma\left(A_{\xi}, \vartheta\right)$ is at least a half-space. Changing if necessary $\theta(t)$ to $\overline{\theta(t)}$ this means that it suffices to verify that there is a neighbourhood $M^{*}$ of $\eta^{*}$ such that

$$
f^{*}\left(\pi_{x} \zeta(0)\right)=0, D f^{*}\left(\pi_{x} \zeta(0)\right)=0
$$

implies that $x=y$ when $x \in l_{y} \cup y$ is close to $y$ and $\zeta(0)^{*} \in M^{*}$. A short computation using (12.6) shows that, putting $\varrho^{2}=\varrho_{1}^{2}+\varrho_{2}^{2},(12.7)$ implies

$$
\begin{aligned}
& -2 x_{1}-\varrho_{1}^{2}+\varrho_{2}^{2}+O\left(\varrho^{3}\right)=0 \\
& -2 x_{1}=\varrho^{2}+O\left(\varrho^{3}\right)=0
\end{aligned}
$$

so that $2 x_{1}+\varrho^{2} \leqslant c^{3} \varrho^{3}$ with some fixed $c$ when $\varrho$ and $x_{1}$ are small enough. Hence $x_{1}=\varrho=0$ or else $\varrho \geqslant c^{-1}$. This proves (ii). The properties (iii) and (iv) follow in the same way (with $N(t)^{*}$ independent of $t$ ) if we can show that $f^{*}\left(\pi_{x} \theta\right)=0$ implies

$$
x_{1}+\varrho\left(\theta^{*}\right)^{2} \leqslant c \varrho\left(\theta^{*}\right)^{3}
$$

for some fixed $c>0$ when $x_{1} \geqslant 0$ and $\varrho\left(\theta^{*}\right) \geqslant 0$ are small enough. But this follows from (12.6). We get, in fact,

$$
(t+s)^{2}\left(x_{1}+\varrho_{1}^{2}\right)+\varrho_{2}^{2}=O(1)(t+s)^{2} \varrho\left(\theta^{*}\right)^{3}
$$

so that the desired inequality results by the definition of $\varrho\left(\theta^{*}\right)$. Hence, by Lemma (11.4), the local Petrovsky condition holds when $p$ is even. 
We shall now prove the converse using the fact that there is a homology class in $H_{n-2}\left(X^{*}, A^{*}\right)$ containing a cycle $e(x)^{*}$ contracting to the point $\eta^{*}$ as $x$ belongs to $l_{y}$ and tends to $y$. This is the vanishing class of the Picard-Lefschetz theory and can be got as follows. By the classical Morse lemma there are analytic coordinates $\xi_{3}^{\prime}=\xi_{3}+\ldots, \ldots, \xi_{n}^{\prime}=\xi_{n}+\ldots$ defined for small $\xi_{3}, \ldots$ such that $Q\left(\xi_{3}, \ldots, \xi_{n}\right)+H^{*}\left(\xi_{3}, \ldots, n_{n}\right)=Q\left(\xi_{3}^{\prime}, \ldots, \xi_{n}^{\prime}\right)$ and hence, if $\xi_{1}=1, f^{*}(\xi)=2 \xi_{2}+Q\left(\xi_{3}^{\prime}, \ldots, \xi_{n}^{\prime}\right)$ so that

$$
f^{*}\left(\pi_{x} \xi\right)=-2 x_{1}-Q\left(\xi_{3}^{\prime}, \ldots, \xi_{n}^{\prime}\right)
$$

Define $e(x)^{*} \subset X^{*}$ by the map $\left(\xi_{3}, \ldots, \xi_{n}\right) \rightarrow \pi_{x} \xi$ where

$$
f^{*}\left(\pi_{x} \xi\right) \leqslant 0, \quad \operatorname{Re} \xi_{3}^{\prime}=\ldots=\operatorname{Re} \xi_{p}^{\prime}=0, \quad \operatorname{Im} \xi_{p+1}^{\prime}=\ldots=0
$$

and orient it in some way. Then $\partial e(x)^{*} \subset A^{*}$ and $e(x)^{*}$ contracts to $\eta^{*}$ as $x_{1} \downarrow 0$. Next, consider $\pi_{x} \zeta(t)^{*} \subset X^{*}-A^{*} \cap X^{*}$ parametrized by

$$
\left(1,-x_{1}, \tau \xi_{3}, \ldots, \tau \xi_{p+1},(\tau+i t)^{-1} \xi_{p+1}, \ldots\right)
$$

with $\tau= \pm 1$ and small real $\xi_{3}, \ldots, \xi_{n}$. This cycle has two sheets that meet only at $\eta^{*}$ and induce equal or opposite orientations of the manifold $\operatorname{Im} \xi_{3} \ldots=\operatorname{Im} \xi_{p}=0, \operatorname{Re} \xi_{p+1}=\ldots=0$ according as $p$ is odd and even. Also, close to $\eta^{*}, \pi_{x} \zeta(t)^{*}$ meets $e(x)^{*}$ only at $\pi_{x} \eta^{*}$. Hence the intersection number $\left(e(x)^{*}, \pi_{x} \zeta(t)^{*}\right)$ equals \pm 2 or 0 according as $p$ is odd or even. Hence, if $p$ is odd, the class of $\pi_{x} \zeta(t)^{*}$ cannot contain a cycle of the form $\pi_{x} \gamma^{*}$ where $\gamma^{*} \subset Y^{*}-Y^{*} \cap A^{*}$. In fact, every such cycle avoids a neighbourhood of $\eta^{*}$ and hence does not intersect $e(x)^{*}$ when $x_{1}$ is small.

\section{\$13. Weak sharpness across plane parts of the wave front surface}

We shall first define sharpness of general distributions across a hyperplane.

Let $M \subset \operatorname{Re} Z^{\prime}$ be an open part of the hyperplane $x_{1}=0$, let $N$ denote neighbourhoods of $M$ in $\operatorname{Re} Z^{\prime}$ and let $N_{ \pm}$be the parts of $N$ where $x_{1}>0$ and $x_{1}<0$ respectively. Let $C^{0 . \infty}(M)$ be the space of all $f \in C^{\infty}\left(N_{+} \cup N_{-}\right)$which have $C^{\infty}$ extensions $f_{ \pm}$from $N_{ \pm}$to $N_{ \pm} \cup M$. Such a function, then, has a $C^{\infty}$ jump $f_{+}\left(0, x^{\prime}\right)-f_{-}\left(0, x^{\prime}\right)$ at $M$. Next, put

$$
C^{-\infty, \infty}(M)=\bigcup_{k} C^{k, \infty}(M)
$$

where $C^{k, \infty}(M)=\left(\partial / \partial x_{1}\right)^{-k} C^{0, \infty}(M)$ with $k$ an integer of arbitrary sign and $\left(\partial / \partial x_{1}\right)^{-k}$ denoting differentiation or integration in the distribution sense. The elements of (13.1) are said to be sharp across $M$. Clearly, $C^{k, \infty}(M) \supset C^{k+1 . \infty}(M)$ is a filtration of the space (13.1) and we have 


$$
D^{\alpha} C^{k, \infty}(M)=C^{k-\alpha_{1}, \infty}(M)
$$

The intersection

$$
C^{\infty, \infty}(M)=\bigcup_{k} C^{k, \infty}(M)
$$

consists of all $C^{\infty}$ functions defined in neighbourhoods of $M$. Note that if $f$ is a bounded function close to $M$ and all derivatives of $f$ of some order $p$ belong to $C^{0 . \infty}\left(N_{+} \cup N_{-}\right)$and have $C^{\infty}$ extensions to $N_{+} \cup M$ and $N_{-} \cup M$, then $f \in C^{0, \infty}(M)$.

The class of $f \in C^{-\infty}{ }^{\infty}(M)$ modulo $C^{\infty} .^{\infty}(M)$ is called the jump of $f$ and is denoted by $J(f)$. It is represented by an asymptotic series.

$$
J(f) \sim \sum^{\infty} \theta_{j}\left(x_{1}\right) f_{j}\left(x^{\prime}\right), x^{\prime}=\left(x_{2}, \ldots, x_{n}\right),
$$

with coefficients $f_{j} \in C^{\infty}(M)$. Here $\theta_{k}$ denotes successive derivatives and integrals of the one-dimensional Heaviside function $\theta(t)=(1+\operatorname{sgn} t) / 2$ so that if $k \geqslant 0$,

$$
\theta_{k}(t)=\theta(t) t^{k} / k !, \quad \theta_{-1-k}(t)=\delta^{(t)}(t) \text {. }
$$

The formula (13.2) has to be taken in the sense that

$$
f-\sum^{k_{c}} \theta_{j}\left(x_{1}\right) f_{j}\left(x^{\prime}\right) \in C^{k+1 . \infty}(M) \text {. }
$$

One verifies that the series is unique, that it vanishes if and only if $f \in C^{\infty}, \infty(M)$ and that

$$
J(Q(D) f)=Q(D) J(f)
$$

for all polynomials $Q$.

When $n=1, M$ is simply the origin of the $x_{1}$-axis and $f \in C^{-\infty} \cdot \infty(0)$ if and only if $f=f\left(x_{1}\right)$ is sharp at 0 from both sides. When $n>1$, this statement is no longer true as shown by the example $f=\delta\left(x_{1}\right) g\left(x^{\prime}\right)$ where $g \neq 0$ is a distribution. In this case it is convenient to introduce also a wider class of distributions $f(x)$ in neighbourhoods of $M$ with the property that

$$
(f, g)\left(x_{1}\right)=\int f\left(x_{1}, x^{\prime}\right) g\left(x^{\prime}\right) d x^{\prime} \in C^{-\infty, \infty}(0)
$$

for all $g \in C_{0}^{\infty}(M)$. We denote this class by $C_{w}^{-\infty, \infty}(M)$ where $w$ stands for "weak". Its elements are said to be weakly sharp across $M$. Clearly, $C_{w}^{-\infty, \infty}(M) \supset C^{-\infty . \infty}(M)$ and the example $f=e^{i\left|x^{\prime}\right| / x_{1}} \in C_{w}^{-\infty, \infty}(M)$ shows that the first space is much bigger than the second. When $f$ if weakly sharp across $M$, the coefficients $\left(f_{j}, g\right)$ of the jump

$$
J((f, g))=\sum^{\infty} \theta_{j}\left(x_{1}\right)\left(f_{j}, g\right)
$$

are distributions in $M$ and (13.2), (13.3) hold in the distribution sense. Of course, if $f \in C^{-\infty, \infty}(M)$ and (13.2) holds in the distribution sense, then the coefficients $f_{f}$ are $C^{\infty}$-functions and (13.3) holds in the strict sense. 


\section{Weak sharpness of fundamental solutions across hyperplanes corresponding to conical points}

Let $a \in \operatorname{Hyp}(\vartheta, m)$ and let $a_{\eta}$ be the localization of $a$ at some $0 \neq \eta \in \operatorname{Re} Z$. The point $\eta^{*}$ is said to be a conical point of $\operatorname{Re} A^{*}$ if the lineality $L\left(A_{\eta}\right)$ is minimal, i.e. if $L\left(A_{\eta}\right)=\mathbf{C} \eta$. It follows that only complete polynomials possess conical points and that $a_{\eta}$ is a complete polynomial on $Z / \mathbf{C} \eta$. If the coordinates are chosen so that $\eta=(1,0, \ldots, 0)$ and $p=m_{\eta}(a)$ is the multiplicity of $\eta$, then $\eta$ is a conical point if and only if $a(\zeta) \equiv$ $\zeta_{1}{ }^{m-p} a_{\eta}\left(\zeta^{\prime}\right) \bmod \zeta_{1}{ }^{m-p-1}$ where $\zeta^{\prime}=\left(\zeta_{2}, \ldots, \zeta_{n}\right)$ and $a_{p}=a_{\eta}$ is a complete polynomial of homogeneity $p$. The dual cone $K\left(A_{\eta}, \vartheta\right)$ is then contained in and spans the hyperplane $x_{1}=0$. The following lemma shows that $E(a, \vartheta, x)$ is weakly sharp across this hyperplane and gives an explicit formula for its jump.

Lemma (13.5). Let $a \in \operatorname{Hyp}(\vartheta, m)$, let $\eta^{*} \in \operatorname{Re} A^{*}$ be a conical point and $a_{\eta}$ the corresponding localization. Choose coordinates such that $\eta=(1,0, \ldots, 0), \vartheta=\left(\vartheta_{1}, \ldots\right)$ with $\vartheta_{1}>0$. Then the distribution

$$
x \rightarrow E(a, \vartheta, x)
$$

is weakly sharp across the hyperplane $x_{1}=0$ and the asymptotic expansion of its jump is

$$
\sum_{j \geqslant 0} \theta_{m-p-1+j}\left(x_{1}\right) H_{p+1-j-n}\left(x^{\prime}\right)
$$

where the indices denote homogeneity,

$$
H_{p+1-j-n}\left(x^{\prime}\right)=\sum_{0 \leqslant l \leqslant j} Q_{j l}\left(D^{\prime}\right) E\left(a_{\eta}^{l+1}, x^{\prime}\right)
$$

and where the $Q_{j l}$ are polynomials given by the formal expansion of $a(\zeta)^{-1}$ in terms of rational functions whose denominators are powers of $\zeta_{1}$ and $a_{\eta}$,

$$
a(\zeta)^{-1}=\sum_{0 \leqslant l \leqslant j} \zeta_{1}^{p-m-j} a_{\eta}\left(\zeta^{\prime}\right)^{-l-1} Q_{j l}\left(\zeta^{\prime}\right)
$$

Before proving this lemma we state a corollary involving hypotheses which imply that (13.6) converges pointwise to the jump $J(a, \vartheta, x)$ of $E(a, \vartheta, x)$ across the hyperplane $x_{1}=0$.

COROLLARY (13.8). Suppose that for all large $k$ and some $y \neq 0$ with $y_{1}=0$, the functions $x \rightarrow E\left(a^{k}, \vartheta, x\right)$ are holomorphically sharp at $y$ from both sides of the hyperplane $x_{1}=0$. Then the asymptotic series (13.6) converges for small $x-y$ to the jump $J(a, \vartheta, x)=\theta_{m-p-1}\left(x_{1}\right) H(x)$ of $E(a, \vartheta, x), H(x)$ being holomorphic at $y$. Note that since $p<m$, the jump is a locally bounded function close to $y$, continuous when $p>m-1$. 
Proof of the corollary. Assume first that $E$ is continuous across $x_{1}=0$ at $y$. Then close to $y$, then jump $J(a, \vartheta, x)$ is the function $\theta\left(x_{1}\right)\left(E_{+}(a, \vartheta, x)-E_{-}(a, \vartheta, x)\right)$ where $E_{ \pm}$denote the holomorphic extensions of $E$ from $x_{1}>0$ and $x_{1}<0$ respectively. The difference $E_{+}-E_{-}$ being holomorphic, a comparison with the weak asymptotic series (13.6) proves the assertion in this case. Now, since $a$ is a complete polynomial, $E\left(a^{k}, \vartheta, \cdot\right)$ is continuous for large $k$. Hence the corollary holds for such powers. In the formula for the jump, this changes $m, p$ to $k m, k p$. Since, by (13.4), $J(a, \vartheta, x)=a^{k-1}(D) J\left(a^{k}, \vartheta, x\right)$ and $a(D) \equiv D_{1}^{m-p} a_{\eta}\left(D^{\prime}\right)$ $\bmod D_{1}^{m-p-1}$, the corollary follows.

Proof of the lemma. We have

$$
a(\zeta)=\zeta_{1}^{m-p}\left(a_{\eta}\left(\zeta^{\prime}\right)-b(\zeta)\right)
$$

where

$$
\zeta^{\prime}=\left(\zeta_{2}, \ldots, \zeta_{\eta}\right)
$$

and

$$
b(\zeta)=\sum_{0<j \leqslant m-p} \zeta_{1}^{-j} b_{j}\left(\zeta^{\prime}\right)
$$

Applying the identity

$$
(A-B)^{-1}=A^{-1}+B A^{-1}+\ldots+B^{N} A^{-N-1}+B^{N+1} A^{-N-1}(A-B)^{-1}
$$

with $A=a_{\eta}, B=b$ gives

$$
a(\zeta)^{-1}=\zeta_{1}^{p-m}\left(a_{\eta}^{-1}+b a_{\eta}^{-2}+\ldots+b^{N} a_{\eta}^{-N-1}\right)+b^{N+1} a_{\eta}^{-N-1} a^{-1} .
$$

Expanding $b$ in terms of $\zeta_{1}$, the first sum om the right equals

and hence, since $\vartheta_{1}>0$,

$$
\zeta_{1}^{p-m} \sum_{0 \leqslant l \leqslant j \leqslant N} Q_{j l}\left(\zeta^{\prime}\right) \zeta_{1}^{-j} a_{\eta}\left(\zeta^{\prime}\right)^{-l-1}
$$

$$
E(a, \vartheta, x)=\sum_{0 \leqslant l \leqslant j \leqslant N} \theta_{m-p-1+j}\left(x_{1}\right) Q_{j l}\left(D^{\prime}\right) E\left(a_{\eta}^{l+1}, \vartheta^{\prime}, x^{\prime}\right)+F(x)
$$

where

$$
F(x)=(2 \pi)^{-n} \int e^{i x \zeta} b(\zeta)^{N+1} a_{\eta}\left(\zeta^{\prime}\right)^{-N-1} a(\zeta)^{-1} d \zeta
$$

with $\zeta=\xi-i \vartheta$. Here the last integrand is majorized by

and hence, if $g \in C_{0}^{\infty}\left(\mathbf{R}^{n-1}\right)$,

$$
O\left(\left|\zeta_{1}\right|^{-N-1}\left|\zeta^{\prime}\right|^{m(N+1)}\right)
$$

$$
\int F(x) g\left(x^{\prime}\right) d x^{\prime}=O\left(\left|x_{1}\right|^{N-1}\right)
$$

Since the terms of (13.9) with $j \geqslant N-m+p$ satisfy the same inequality, the lemma is proved.

13 - 732907 Acta mathematica 131. Imprimé le 11 Décembre 1973 


\section{§ 14. Holomorphic sharpness across plane parts of the wave front surface}

We shall now state and prove the main result of this chapter. Recall that if $a \in \operatorname{Hyp}(\vartheta)$, the reduced wave front surface $\hat{W}(A, \vartheta)$ is the union of the local propagation cones $K\left(A_{\xi}, \vartheta\right)$ for $\xi$ real not in $L(A)$.

THЕ ОR Мм (14.1). Let $a \in \operatorname{Hyp}(\vartheta)$, let $0 \neq \eta \in \operatorname{Re} A$ be a conical point and let

$$
y \in K\left(A_{\eta}, \vartheta\right)-W\left(A_{\eta}, \vartheta\right)
$$

be a simple point of $W(A, \vartheta)$. Then, for all $k>0, E\left(a^{k}, \vartheta, \cdot\right)$ is holomorphically sharp at y from both sides of $W(A, \vartheta)$.

Note (14.3). The theorem is almost immediate when $n=3$. In fact, then (14.2) means that the line $\operatorname{Re} Y^{*}$ does not touch any branch of $\operatorname{Re} A^{*}$ (or $A^{*}$ ) at $\eta^{*}$. When $x$ approaches $y$ in such a way that $\operatorname{Re} X^{*}$ has the same property then, since $y$ is simple, the non-real part of $A^{*} \cap X^{*}$ stays away from $\operatorname{Re} X^{*}$ while som of its real points collapse. Hence the assertion of the theorem follows from Example (10.3).

Proof. We are going to verify the hypotheses of Lemma 11.4. Let us choose coordinates so that

$$
\eta=(1,0, \ldots, 0), \quad y=(0,1,0, \ldots, 0), \quad \vartheta=(1,1,0, \ldots, 0)
$$

Then, close to $y, x_{1}=0$ is the equation of $K\left(A_{\eta}, \vartheta\right)$. We let $l_{y}^{\varepsilon}$ be the lines $x=\left(x_{1}, 1,0, \ldots, 0\right)$ with $\operatorname{sgn} x_{1}=\varepsilon$ and $x_{1}$ small and let $L^{\varepsilon}$ be the corresponding component of the real complement of $W(A, \vartheta)$. The projection $\pi_{x}: Y \rightarrow X$ is defined by $\pi_{x} \xi=\xi-(x, \xi)(y, \lambda)^{-1} \lambda$ where $\lambda=(0,1,0, \ldots)$. In particular,

$$
x \in l_{y}^{\varepsilon}, \xi \in Y \Rightarrow \pi_{x} \xi=\left(\xi_{1},-x_{1}, \xi_{3}, \ldots, \xi_{n}\right)
$$

We shall now choose the chains $\zeta(t), \theta(t) \subset Y$ of Lemma 11.4. We put

$$
\zeta(t)=\xi-i t \chi, \theta(t)=\xi-i t \omega
$$

with $t>0$ and $\xi, \chi, \omega$ real and depending on certain parameters. The chain $\zeta(0)=\xi$ should contain $\pm \eta$ and be transversal to rays through the origin. We put $\xi=(\tau, 0, u)$ where $\tau= \pm 1, u=\left(u_{3}, \ldots, u_{n}\right) \in \mathbf{R}^{n-2}$. We should now have $\chi \in \Gamma\left(A_{\xi}, \vartheta\right)$ when $\xi$ is close to $\pm \eta$. Since $a$ and $A$ are approximated at $\eta$ by their localizations $b=a_{\eta}$ and $B=A_{\eta}$, a reasonable choice of $\chi$ is to take real homogeneous $C^{\infty}$ map $u \rightarrow v(u) \in \mathbf{R}^{n-2}$ such that

$$
\chi=(*, 0, v(u)) \in \Gamma\left(B_{\xi}, \vartheta\right)
$$


for all $u$. By virtue of (14.2) this is possible and, since $a_{\eta}$ is independent of the first coordinate, we can here replace the star by any number. We now put $*=-\alpha \varepsilon$ and, for technical reasons, replace $u$ by $\varrho u$ where $|u|=\left(u_{3}^{2}+\ldots+u_{n}^{2}\right)^{\frac{1}{2}}=1$ and $\varrho \geqslant 0$. This gives

$$
\pi_{x} \xi=\left(\tau,-x_{1} \tau, \varrho u\right) \quad \pi_{x} \chi=\left(-\alpha \varepsilon,\left|x_{1}\right| \alpha, \varrho v(u)\right)
$$

where, since $(0,1,0, \ldots, 0) \in \Gamma(B, \vartheta)$

$$
\left|x_{1}\right|+\varrho>0 \Rightarrow \pi_{x} \chi \in \Gamma\left(B_{\pi_{x} \xi}, \vartheta\right)
$$

if $\alpha>0$. Further, if $\alpha \rightarrow \infty, \zeta(t)^{*}$ contracts to the point $\left(-\varepsilon,\left|x_{1}\right|, 0, \ldots, 0\right)^{*}$ which is a good reason for constructing $\theta(t)$ from $\zeta(t)$ by replacing the parameter $\alpha$ by a variable $\beta>\alpha$. Finally, we shall put

$$
\zeta(t)=(\tau+i \propto t \varepsilon, 0, \varrho(u-i t v(u)))
$$

where $\tau= \pm 1, \varrho \geqslant 0, u \in \mathbf{R}^{n-2}$ with $|u|=1$ are variables and $t>0$ and $\alpha>0$ parameters at our disposal. Also, with the same $t$ and $\alpha$,

$$
\theta(t)=(\tau+i \beta t \varepsilon, 0, \varrho(u-i t v(u)))
$$

where the variables are $\tau, \varrho, u, \beta>\alpha$. In all this,

$$
(0,0, v(u)) \in \Gamma\left(B_{\xi}, \vartheta\right), \xi=(\tau, 0, u),
$$

for all $\tau$ and $u$. Then, with $I=\{\tau ; \tau= \pm 1\}$,

$$
\zeta(t): I \times R^{n-2} \rightarrow Y, \quad \theta(t): I \times R^{n-2} \times\{\beta ; \beta>\alpha\} \rightarrow Y
$$

are orientable manifolds as required by Lemma 11.4 and $\partial \theta(t)=\zeta(t)$. The verification that they have all the desired properties (i) to (iv) of this lemma depends on another lemma to be stated now and proved later. Let $p$ be the homogeneity of the localization $a_{\eta}$. We introduce the polynomial

$$
h(s, u, z)=z^{-p} a(1, z s, z u)
$$

and factor off those zeros of $s \rightarrow h(s, u, z)$ which are close to the zeros of $s \rightarrow a_{\eta}(s, u)$ when $z \in \mathbf{C}$ is small. We get

$$
h(s, u, z)=\prod_{1}^{p}\left(s+\lambda_{k}(u, z)\right) H(s, u, z)
$$

when $z, z s$ are small and $u$ bounded. Here $\lambda_{1}, \ldots, \lambda_{p}, H$ are continuous functions and $H \neq 0$. This gives

$$
a\left(\xi_{1}, \xi_{2}, \varrho u\right)=\prod_{1}^{p}\left(\xi_{2}+\varrho \lambda_{k}(u, z)\right) \xi_{1}^{m-p} H\left(\xi_{2} / \xi_{1}, u, z\right)
$$


where $z=\varrho / \xi_{1}$ and $\xi_{2} / \xi_{1}$ are small and $u$ is bounded. In the lemma that follows, $u, v(u)$ are as in (14.4).

Lemma (14.8). Consider the functions $t, z, u \rightarrow w=\lambda_{k}(u+t v, z)$ when $\operatorname{Im} t \neq 0$ and $t, z \in \mathbf{C}$ are small. There are positive numbers $c, c_{1}, c_{2}$ such that

$$
\begin{gathered}
|t| \leqslant c,|z| \leqslant c,|\operatorname{Im} z| \leqslant c|\operatorname{Im} t| \Rightarrow|\operatorname{Im} w| \leqslant c_{1}|\operatorname{Im} t| \\
\text { same hypothesis and }|\operatorname{Re} w| \leqslant c \Rightarrow \operatorname{Im} w / \operatorname{Im} t \geqslant c_{2} .
\end{gathered}
$$

If a function $t \rightarrow z(t)$ is small at the origin, analytic in $t$ and real for real argument, then the functions $t \rightarrow w(t)=\lambda_{k}(u+t v, z(t))$ are differentiable at the origin and hence, if $z=z(t)$ also satisfies the above hypotheses, then $D \operatorname{Im} w(0)$, where $D=-i d / d t$, is majorized according to (14.9) and (14.10).

Verification of the hypotheses of Lemma 11.4 (i) Obvious. (iii) The last part is obvious since $\zeta(t)=\partial \theta(t)$. Factoring according to $(14.7)$ we get

where

$$
a\left(\pi_{x} \zeta(t)=\left(\prod_{1}^{p} \Lambda_{k}\right)(\tau+i \alpha t \varepsilon)^{m-p} H\left(-x_{1}, u, z\right)\right.
$$

$$
\Lambda_{k}=-x_{1}(\tau+i t \alpha \varepsilon)+\varrho \lambda_{k}(u-i t v, z), z=\varrho(\tau+i t \alpha \varepsilon)^{-1}
$$

so that

$\operatorname{Re} \Lambda_{k}=-x_{1} \tau+\varrho \operatorname{Re} \lambda_{k}, \operatorname{Im} \Lambda_{k}=-t\left|x_{1}\right| \alpha+\varrho \operatorname{Im} \lambda_{k}$.

Since $|z| \leqslant \varrho$ and $|\operatorname{Im} z| \leqslant \varrho \alpha|t|,(14.9)$ and (14.10) of the lemma show that, if $\alpha \geqslant 1$,

$$
0<t \leqslant c, \varrho \alpha \leqslant c,\left|x_{1}\right| \leqslant c \Rightarrow\left|\operatorname{Im} \lambda_{k}\right| \leqslant c_{1} t,
$$

same hypothesis and $\left|\operatorname{Re} \lambda_{k}\right| \leqslant c \Rightarrow \operatorname{Im} \lambda_{k} \leqslant-c_{2} t$.

Hence, under the hypotheses of (14.12), we have the logical chain

$$
\Lambda_{k}=0 \Rightarrow\left|\operatorname{Re} \lambda_{k}\right|>c \Rightarrow\left|x_{1}\right| \geqslant c \varrho \Rightarrow \operatorname{Im} \Lambda_{k} \leqslant-\max \left(\left|x_{1}\right|, c \varrho\right) \alpha t+c_{1} \varrho t .
$$

But the chain is contradictory when $c \alpha>c_{1}$ and $\left|x_{1}\right|+\varrho>0$. Hence there is an $\alpha>1$, e.g. $\alpha=\left(c_{1}+c\right) / c$, such that $a\left(\pi_{x} \zeta(t)\right) \neq 0$ when $\left|x_{1}\right|+\varrho>0$ and $t>0$ are small enough. This proves (iii).-(iv) Repeat the same arguments with $\alpha>1$ fixed as above but now also with some fixed small enough $t>0$ and with $z=\varrho(\tau+i \beta \varepsilon t)^{-1}$. Then $|\operatorname{Im} z| \leqslant c t$ is equivalent to $\varrho \beta\left(1+\beta^{2} t^{2}\right)^{-1} \leqslant c$ and this is true for all $\beta>0$ if, e.g. $\varrho \leqslant c t$. We arrive at the conclusion that $a\left(\pi_{x} \theta(t)\right) \neq 0$ for every fixed small enough $t>0$ when $\left|x_{1}\right|+\varrho>0$ is small enough depending on $t$. This proves (iv).-(ii) Let $\alpha>1$ be as under (iii), let $\beta \geqslant 0$ and $\vartheta^{\prime}=(0,1,0, \ldots, 0)$ and consider 


$$
\zeta_{x}(t)=\pi_{x} \zeta(t)-i t \beta \vartheta^{\prime}
$$

Factoring according to (14.7) we get, when $t>0$ and $z, \beta t$ are small

$$
a\left(\zeta_{x}(t)\right)=\prod_{1}^{p}\left(\Lambda_{k}-i t \beta\right)(\tau+i t \alpha \varepsilon)^{m-p} H(-*, u-i t v, z)
$$

where $*=-x_{1}-i \beta t(\tau+i t \alpha \varepsilon)^{-1}$ and $\Lambda_{k}, z$ are as in (14.10). By the lemma, the functions $t \rightarrow \Lambda_{k}-i t \beta$ are differentiable for $t=0$ and (14.12), (14.13) show that

$$
\varrho \alpha \leqslant c,\left|x_{1}\right| \leqslant c \Rightarrow\left|D \operatorname{Im} \lambda_{k}\right| \leqslant c_{1}
$$

same hypothesis and $\left|\operatorname{Re} \lambda_{k}\right| \leqslant c \Rightarrow D i \operatorname{Im} \lambda_{k} \geqslant c_{2}$

where $D=-i d / d t$ taken at $t=0$. We claim that

$$
\Lambda_{k}-i t \beta=0 \Rightarrow D\left(\Lambda_{k}-i t \beta\right)>0
$$

when $\left|x_{1}\right|+\varrho>0$ is small enough. In fact,

$$
D\left(\Lambda_{k}-i t \beta\right)=\left|x_{1}\right| \alpha+\beta+D i \operatorname{Im} \lambda_{k}
$$

is positive when the last term is positive and hence, in view of (14.15), we need only consider the case $\left|\operatorname{Re} \lambda_{k}\right|>c, \Lambda_{k}-i t \beta=0$, in particular $\left|x_{1}\right|=\varrho\left|\operatorname{Re} \lambda_{k}\right| \geqslant \varrho c$. But then, by (14.14),

$$
D\left(\Lambda_{k}-i t \beta\right) \geqslant \max \left(\left|x_{1}\right|, c \varrho\right) \alpha+\beta-c_{1} \varrho,
$$

which, by our choice of $\alpha$ is positive for all $\beta \geqslant 0$. Now by (14.16), the degree at zero of the polynomial $t \rightarrow a\left(\zeta_{x}(t)\right)$ is independent of the choice of $\beta$ and, when $\beta$ is large,

$$
-\operatorname{Im} \zeta_{x}(t)=t\left(-\alpha \varepsilon,\left|x_{1}\right| \alpha+\varrho, \varrho v(u)\right)
$$

belongs to $\Gamma\left(A_{\eta}, \vartheta\right)$ and hence also to $\Gamma\left(A_{\pi_{x} \xi}, \vartheta\right)$ when $\left|x_{1}\right|+\varrho$ is small. It follows that, putting

$$
\chi=D \zeta(0)=(-\alpha \varepsilon, 0, \varrho v(u)), \xi=(\tau, 0, \varrho u),
$$

then $\pi_{x} \chi \in \Gamma\left(A_{\pi_{x} \xi}, \vartheta\right)$ when $\left|x_{1}\right|+\varrho>0$ is small enough and this proves (ii).

Proof of Lemma 14.8. Since $a \in \mathrm{Hyp}(\vartheta), h$ as defined by (14.5) belongs to $\operatorname{Hyp}_{1 \mathrm{loc}}\left(\vartheta^{\prime}\right), \vartheta^{\prime}=(1,0, \ldots, 0)$ and $(14.6)$ gives

$$
h(s, u+t v, z)=\prod_{1}^{p}\left(s+\lambda_{x}(u+t v, z)\right) \cdot H(s, u+t v, z)
$$

when $s, z s, t$ are small enough.Hence (14.9) follows from Corollary 15.16. To prove (14.10) 
we shall use Theorem 15.19. Let $\xi=(0, u, 0)$ and define $S$ by $|u|=1$, $u$ real, put $\zeta=(s, 0, z)$ and

$$
K_{\xi}=\left\{\left((\operatorname{Im} s)_{+}, v(u), 0\right) \in \Gamma\left(T_{\xi} P h, \vartheta^{\prime}\right)\right\} .
$$

Then, by the theorem quoted,

$$
h(\varrho s, \varrho z, \varrho(u+t v)) \neq 0
$$

when $\varrho>0, s, z, t,(\operatorname{Im} s)_{-} / \operatorname{Im} t$ and $\operatorname{Im} z / \operatorname{Im} t$ are small enough. Now, by the definition of $h$, (14.17) is equivalent to $h\left(s, \varrho^{2} z, u+t v\right) \neq 0$ and hence it holds with $\varrho=1$ when $s, z, t$, $(\operatorname{Im} s)_{-} / \operatorname{Im} t, \operatorname{Im} z / \operatorname{Im} t$ are small enough. But this is just another way of stating (14.10). To prove the last part of the lemma we remark that the functions $t \rightarrow w(t)$ can be developed in Puiseux series for small $t$ and that if all these series are real for real argument, they must be power series.

This finishes the proof of Theorem 14.1 and we proceed to an important corollary.

THEOREM (14.18). (a) Under the hypotheses of Theorem 14.1, let $p$ be the homogeneity of $a_{\eta}$. Then the jump $J\left(a^{k}, \vartheta, x\right)$ of $E\left(a^{k}, \vartheta, x\right)$ at y across $W(A, \vartheta)$ equals $\theta_{k(m-p)-1}(\eta x) H_{k p+1-n}(x)$ where the indices indicate homogeneity and $H$ is holomorphic.

(b) Suppose in addition that the Petrovsky condition for $a_{\eta}$ holds at $y$, namely

$$
\beta\left(A_{\eta}, y, \vartheta\right)^{*}=0 \text { in } H_{n-3}\left(Y^{*}-Y^{*} \cap A_{\eta}^{*}\right)
$$

where $a_{\eta}$ is considered as a polynomial in $Z \bmod \mathbf{C} \eta$. Then $H_{k p+1-n}(x)$ is a polynomial. In particular, if $k p<n-1, E\left(a^{k}, \vartheta, \cdot\right)$ is holomorphic across $W(A, \vartheta)$ at $y$ and if $Q(\xi)$ is a homogeneous polynomial vanishing $q$ times when $\xi=\eta$ and if $q>k p+1-n$, then $Q(D) E\left(a^{k}, \vartheta, \cdot\right)$ is holomorphic across $W(A, \vartheta)$ at $y$.

Proof. (a) This is just a restatement of Corollary 13.8 whose ssumption holds by virtue of Theorem 14.1.

(b) Since the Petrovsky condition (14.19) holds, the fundamental solutions $E\left(a_{\eta}^{l+1}, x^{\prime}, \vartheta^{\prime}\right)$ (notation of Lemma 13.5) are polynomials near $y^{\prime}$ so that, by virtue of (13.6), (13.7), $L_{p+1-n}(x)$ is a polynomial. The same argument works for powers of $a$.

\section{Application to hyperbolic first-order symmetric systems}

Put $B(\xi)=\Sigma_{1}^{n} B_{i} \xi_{i}$ where the $B_{i}$ are hermitian $m \times m$ matrices, let $\vartheta \in \operatorname{Re} Z$ and assume that $B(\vartheta)>0$ is positive definite. Then $a(\xi)=\operatorname{det} B(\xi)$ belongs to Hyp $(\vartheta, m)$ and the fundamental solution of $B(D)$ with support in $K(A, \vartheta)$ is $E(B, \vartheta, x)=C(D) E(a, \vartheta, x)$ where $C(\xi)$ is the matrix of minors of $B(\xi)$. The homogeneity of $C(\xi)$ is $m-1$ and, by 
elementary spectral theory, when $\eta$ is so close to $\vartheta$ that $B(\eta)>0$ and if $t \rightarrow a(t \eta+\xi)$ vanishes $k+1$ times for some $t$, then $t \rightarrow C(t \eta+\xi)$ vanishes at least $k$ times for the same value of $t$. Hence this is true independently of $t$. In particular, if $a, y, \eta, \beta^{*}=\beta\left(A_{\eta}, y, \vartheta\right)^{*}$ meet the requirements of (b) of the preceding theorem and $Q(\xi)$ is an entry of $C(\xi)$, then $q \geqslant p-1>p+1-n$ when $n>2$. Hence, if $n>2, E(B, \vartheta, x)$ is holomorphic across $W(A, \vartheta)$ at $y$. When $n=3$, the condition on $\beta^{*}$ is automatic so that in this case $E(B, \vartheta, x)$ is holomorphic across all plane parts of $W(A, \vartheta)=W(\operatorname{det} B, \vartheta)$. Hence we have the inclusion from left to right, first observed by Bazer and Yen [4] of the following

THEOREM (14.20). Let $B=\Sigma_{1}^{3} B_{i} \xi_{i}$ be as above and let $a(\xi)=\operatorname{det} B(\xi)$. Then

$$
\operatorname{SSE}(B, \vartheta, \cdot)=\text { closure of } \bigcup_{n_{\xi}(a)=1} K\left(A_{\xi}, \vartheta\right) \text {. }
$$

Here $n_{\xi}(a)$ is the reduced dimension of $a_{\xi}$ so that the right side is indeed $W(\operatorname{det} B, \vartheta)$ minus its relatively open plane parts. That the left side cannot be smaller than the right side is an easy consequence of the Localization Theorem I.4.10. The details are left to the reader.

\section{\$ 15. Local hyperbolicity}

A homogeneous polynomial $a(\xi)$ is, by definition, hyperbolic with respect to $\vartheta \in \mathbf{R}^{n}$ if $a(\vartheta) \neq 0$ and the equation $a(\xi+t \vartheta)=0$ has only real roots $t$ for real $\xi$. We shall now study the situation when only all the small roots, i.e. those tending to zero with $\xi$, are required to be real. This gives us the notion of local hyperbolicity. We shall study it here for its own sake and in order to prove two results (Corollary 15.16 and Theorem 15.19 below) that have been used in the preceding paragraph. The basic definitions are as follows.

Definition (15.1). Let $\mathcal{A}$ be the space of functions $h(\xi), \xi \in \mathbf{C}^{n}$, analytic at the origin and, when $h \in \mathcal{A}$, define its principal part $P h$ as the first non-vanishing term $h_{m}$ in the expansion $h=\Sigma h_{k}$ of $h$ in terms of polynomials $h_{k}$ of homogeneity $k$. The number $m$ is also called the degree of $h$ (at zero). An $h \in \mathcal{A}$ is said to be locally hyperbolic with respect to $\vartheta \in \mathbf{R}^{n}$ if

$$
\xi \in R^{n}, \operatorname{Im} t \neq 0 \Rightarrow h(\xi+t \vartheta) \neq 0
$$

when $\xi, t$ are small enough. When the degree of $h$ is $m$, the class of these functions will be denoted by $\mathrm{Hyp}_{\text {loc }}(\vartheta, m)$ and by $\mathrm{Hyp}_{\text {loc }}(\vartheta)$ when the degree is not specified.

We shall see later that (15.1) implies $\left(^{1}\right)$

$$
P h(\vartheta) \neq 0 .
$$

(1) This was pointed out to us in May 1973 by T. Kawai and M. Kashiwara. Our original definition required both (15.1) and (15.2). 
If $h$ is a homogeneous polynomial, (15.2) holds without restriction if it holds for small $\xi, t$ and says that $h$ is hyperbolic with respect to $\vartheta$, i.e. $h \in$ Hyp $(\vartheta)$. Hence Нyp $(\vartheta)$ and Hyp $(\vartheta, m)$ are precisely the homogeneous elements of $\mathrm{Hyp}_{\mathrm{loc}}(\vartheta)$ and $\mathrm{Hyp}_{\mathrm{loc}}(\vartheta, m)$ respectively. Since $r^{-m} h(r(\xi+t \vartheta))$ tends to $P h(\xi+t \vartheta)$ when $r \rightarrow 0$ and $m$ is the degree of $h$, (15.2) shows that

$$
h \in \mathrm{Hyp}_{\text {loc }}(\vartheta) \Rightarrow P h \in \mathrm{Hyp}(\vartheta)
$$

We shall now put

$$
\Gamma(h, \vartheta)=\Gamma(P h, \vartheta)
$$

where, by Definition I.3.14 and I.3.21, the right side is the component of the real complement of the real hypersurface $P h(\xi)=0$ that contains $\vartheta$.

Note. Local hyperbolicity has also been considered by K. G. Andersson (1971). His definition is essentially the same and he proved the Main Lemma below.

\section{The continuity lemma}

All the properties of locally hyperbolic functions that we shall state and prove depend on two elementary facts: (1) the small zeros of a convergent power series $f$ in one variable are continuous functions of $f$ when one of its coefficients stays away from zero and the preceding ones are small (2) a Puiseux series which is real for real argument is a power series.

We shall first use the second fact to prove (15.2). Fix $\xi \in \mathbf{R}^{n}$ such that $P h(\xi) \neq 0$ and consider the function $f(t, s)=h(t \vartheta+s \xi)$ of two small complex variables $t$ and $s$. Since, by (15.1), $f(t, 0)$ does not vanish identically, $f(t, 0)=c t^{m}(1+O(t))$ for some $c \neq 0$ and some integer $m$ and hence there is a factorization $f(t, s)=F(t, s) \prod_{1}^{m}\left(t+\lambda_{k}(s)\right)$ where $F$ is analytic at the origin, $F(0,0)=c$ and the $\lambda_{k}(s)$ are Puiseux series without constant terms. By virtue of (15.1) they are real for real $s$ and hence they are ordinary power series, $\lambda_{k}(s)=O(s)$. This shows that the degree of $f(t, s)$ is $m$ so that $P(t, s)=P h(t \vartheta+s \xi)$. In particular, $P h(\vartheta)=P f(1,0)=c \neq 0$.

Both facts above will now be employed to prove a lemma that will be used many times later on.

Contin Uity Lemma (15.3). Let $s, t \in \mathbf{C}, u \in \mathbf{R}^{M}, v \in \mathbf{R}^{N}$. Suppose that $f(s, t, u, v)$ is analytic in $s, t$ and continuous when $s, t, u$ are small and $v$ belongs to some connected compact part $K$ of $\mathbf{R}^{N}$. Suppose that

(i) the degree of $t \rightarrow f(0, t, 0, v)$ at the origin is constant for all $v \in K$.

(ii) $\operatorname{Im} s \operatorname{Im} t \geqslant 0, \operatorname{Im} s \neq 0 \Rightarrow f(s, t, 0, v) \neq 0$ when $s, t$ are small enough and $v \in K$ 
(iii) $\operatorname{Im} s \neq 0, \operatorname{Im} t=0 \Rightarrow f(s, t, u, v) \neq 0$ Then when $s, t, u$ are small enough and $v \in K$.

(iv) $\operatorname{Im} s \operatorname{Im} t \geqslant 0, \operatorname{Im}(s+t) \neq 0 \Rightarrow f(s, t, u, v) \neq 0$

(v) $s \rightarrow f(s, 0, u, v)$ and $t \rightarrow f(0, t, u, v)$ have the same degree at the origin when $s, t, u$ are small enough and $v \in K$.

Note. When $f(0,0,0, v) \neq 0$ for all $v$ in $K$, the conditions are fulfilled but the conclusions are void.

Proof. According to (i), $f(0, t, 0, v)=c_{p}(v) t^{p}+$ higher terms where $p \geqslant 0$ is an integer and $\left|c_{p}(v)\right|$ has a positive lower bound on $K$. Hence the analytic function $t \rightarrow f(s, t, u, v)$ has precisely $p$ zeros $t=-\lambda_{1}, \ldots,-\lambda_{p}$, continuous functions of $s, u, v$ that tend to zero with $s, u$, uniformly when $v \in K$. Hence

$$
f(s, t, u, v)=c_{p}(v) \prod_{1}^{p}\left(t+\lambda_{k}(s, u, v)\right) F(s, t, u, v)
$$

where $\lambda_{1}, \ldots, \lambda_{p}, F$ are continuous for small arguments and $F(0,0,0,0)=1$. According to (ii),

$$
\operatorname{Im} s>0 \Rightarrow \operatorname{Im} \lambda_{k}(s, 0, v)>0,1 \leqslant k \leqslant p,
$$

when $v \in K$ and $s$ is small and according to (iii),

$$
\operatorname{Im} s>0 \Rightarrow \operatorname{Im} \lambda_{k}(s, u, v) \neq 0,1 \leqslant k \leqslant p,
$$

when $v \in K$ and $s, u$ are small. In other words, when $s, u$ are small and $\operatorname{Im} s>0$, none of the numbers $\lambda_{1}, \ldots, \lambda_{p}$ cross the real axis and when $u=0$, they all lie in the upper half-plane. Hence they are always in the upper half-plane. The same argument with Im $s<0$ also works and hence

$$
\operatorname{sgn} \operatorname{Im} \lambda_{k}(s, u, v)=\operatorname{sgn} \operatorname{Im} s
$$

for all $s, u, v, k$ when $\operatorname{Im} s \neq 0$ and $s, u$ are small enough. Combining (15.4) and (15.5) we get (iv). By classical function theory, the functions $s \rightarrow \lambda_{k}(s, u, v)$ have convergent Puiseux series expansions

$$
\lambda_{k}(s, u, v)=\sum_{0}^{\infty} c_{k j} s^{j / p}, \quad 1 \leqslant \mathrm{k} \leqslant \mathrm{p}
$$

when $v \in K$ and $s, u$ are small enough. Some reflection shows that (15.5) and (15.6) are consistent if and only if

$$
\lambda_{k}(s, u, v)=\lambda_{k}(0, u, v)+c_{k}(u, v) s+O\left(s^{2}\right)
$$

where all $c_{k}>0$. Inserting this into (15.4) proves (v). More precisely, the degree at the origin of $t \rightarrow f(t, 0, u, v)$ and $s \rightarrow f(0, s, u, v)$ equals the number of vanishing $\lambda_{k}(0, u, v)$. 


\section{The main theorem}

The most important properties of locally hyperbolic functions follow from the continuity lemma. We shall first prove

MaIn Lemma (15.7). Let $h \in \mathrm{Hyp}_{\mathrm{loc}}(\vartheta)$ and let $K$ be compact part or $\Gamma(h, \vartheta)$. Then

$$
\begin{gathered}
\xi \in \mathbf{R}^{n}, \eta \in K, \operatorname{Im} s \operatorname{Im} t \geqslant 0, \operatorname{Im}(s+t) \neq 0 \Rightarrow h(\xi+s \vartheta+t \eta) \neq 0 \\
s \rightarrow h(\xi+s \vartheta) \text { and } t \rightarrow h(\xi+t \eta) \text { have the same degree at zero }
\end{gathered}
$$

when $\xi, s, t$ are small enough. When $h=P h$, these statements hold without restriction.

Proof. Let $h$ have the degree $m$. The function

$$
f(s, t, u, v)=r^{-m} h(r(\xi+s \vartheta+t \eta)),
$$

where $u=r, \xi$ and $v=\eta$, satisfies the requirements of the continuity lemma. In fact, since $\Gamma(h, \vartheta)$ is connected, $K$ can be assumed to be connected,

$$
f(0, t, 0, v)=P h(t \eta)=t^{m} P h(\eta)
$$

shows that (i) holds, (iii) holds by definition and (ii) since

$$
f(s, t, 0, v)=\operatorname{Ph}(s \vartheta+t \eta)=\operatorname{Ph}(\vartheta) \prod_{1}^{m}\left(s+t \lambda_{k}(\eta, \vartheta)\right)
$$

where, by the definition of $\Gamma(P h, \vartheta)$, all numbers $\lambda_{k}(\eta, \vartheta)$ are positive. The last statement of the lemma follows since $P h$ is homogeneous.

We can now state and prove the main result. When $h(\zeta)$ is a function defined close to $\xi \in \mathbb{C}^{n}$, we denote by $T_{\xi} h$ the function $h$ transported to 0 , i.e. the function

$$
\eta \rightarrow T_{\xi} h(\eta)=h(\xi+\eta)
$$

defined in a neighbourhood of the origin. When $h$ is a homogeneous polynomial, its localization at $\xi$ as defined in Part I (Definition 3.36) is then $h_{\xi}=P T_{\xi} h$.

MaIN Th EOREM (15.10). Let $h \in \mathrm{Hyp}_{\mathrm{Ioc}}(\vartheta)$. Then $\Gamma(h, \vartheta)$ is an open convex cone containing $\vartheta$ and, if $K$ is a compact part of $\Gamma(h, \vartheta)$, then

$$
\begin{gathered}
\eta \in K \Rightarrow T_{\xi} h \in \mathrm{Hyp}_{\mathrm{loc}}(\eta) \\
\Gamma\left(T_{\xi} h, \vartheta\right) \supset K
\end{gathered}
$$

when $\xi \in \mathbf{R}^{n}$ is small enough. If $h=P h$ is homogeneous, these statements hold without restriction. 
Note. The statement about $\Gamma(h, \vartheta)=\Gamma(P h, \vartheta)$ is of course part of the theory of hyperbolic polynomials of Part I.

Proof. That $\operatorname{Im} t \neq 0 \Rightarrow h(\xi+\zeta+t \eta) \neq 0$ when $\eta \in K$ and when $\xi, \zeta \in \mathbf{R}^{n}$ and $t$ are small enough follows from (15.8) and (15.9) shows that $P T_{\xi} h(\eta) \neq 0$ on $K$ when $K$ contains $\vartheta$. Hence, since $K$ can be chosen to have a non-empty interior, the degree at 0 of $t \rightarrow h(\xi+t \eta)$ is also the degree of $T_{\xi} h$. Hence (15.11) and (15.12) follow. When $h$ is homogeneous, both these statements are of course valid without restriction. The formula (15.8) applied with $\xi=0$ and $h$ replaced by $P h$ shows that $\Gamma(h, \vartheta)$ is star-shaped with respect to $\vartheta$ and hence according to (15.11) with respect to all its points and hence convex. This finishes the proof.

\section{Two corollaries}

Before proceding further we shall give two corollaries of the Main Theorem. The first one uses a slight variant of the definition of inner and outer continuity (Part I, p. 151) which we shall employ also later in this paragraph. A function $\tau \rightarrow C_{\tau}$ from a topological space to open sets in $\mathbf{R}^{n}$ is said to be inner continuous at $\tau_{0}$ if every compact part $K$ of $\mathbf{R}^{n}$ contained in $C_{\tau_{0}}$ is also contained in $C_{\tau}$ when $\tau$ is close enough to $\tau_{0}$. When the sets $C_{\tau}$ are compact, the function is said to be outer continuous at $\tau_{0}$ if every open part $N$ of $\mathbf{R}^{n}$ containing $C_{\tau_{0}}$ also contains $C_{\tau}$ when $\tau$ is close enough to $\tau_{\mathbf{0}}$. Our first corollary is

Corollary (15.13). When $h \in \operatorname{Hyp}_{\text {loc }}(\vartheta)$, the function

$$
\mathbf{R}^{n} \ni \xi \rightarrow \Gamma\left(T_{\xi} h, \vartheta\right)
$$

is inner continuous for all sufficiently small $\xi$. When $h=P h$, this is true without restriction.

Note. The last statement is identical with Lemma 5.9 of Part $I$ in the special case and when $a=b$. The key lemmas 5.1 and 5.9 of Part $\mathrm{I}$ are in fact consequences of the continuity lemma.

Proof. According to (15.12), the function (15.14) is inner continuous at the origin and hence by (15.11) for all sufficiently small $\xi$. The proof of the last statement is left to the reader.

Our second corollary has to do with the factorization

$$
h(\xi+t \eta)=P h(\eta) \prod_{1}^{m}\left(t+\lambda_{k}(\zeta, \eta)\right) H(t, \xi, \eta)
$$

where $h \in \operatorname{Hyp}_{\text {loc }}(\vartheta)$ has degree $m$ and $\eta \in \Gamma(P h, \vartheta)$. A special case of it (when $\eta=\vartheta$ ) has been used by Bony and Schapira [5] who deduce it from a theorem by Kashiwara. 
Corollary (15.16). Factor $h \in \mathrm{Hyp}_{100}(\vartheta, m)$ according to (15.15). Then

$$
\mathrm{C}_{\ni} \xi \rightarrow 0 \Rightarrow \operatorname{Im} \lambda_{k}(\xi, \eta)=O(|\operatorname{Im} \xi|)
$$

locally uniformly when $\eta \in \Gamma(P h, \vartheta)$.

Proof. According to (15.11),

$$
h(\xi+i t \eta)=h(\operatorname{Re} \xi+i(t \eta+\operatorname{Im} \xi)) \neq 0
$$

when $\xi, t$ are small enough, $t \neq 0$ is real and $\eta+t^{-1} \operatorname{Im} \xi$ belongs to a given compact part of $\Gamma(P h, \vartheta)$.

\section{A sharpening}

We shall now sharpen the Main Theorem (15.7) by permitting $\eta$ to depend on $\xi$.

Lемма (15.17). Let $h \in \mathrm{Hyp}_{100}(\vartheta)$, let $S \subset \mathbf{R}^{n}-\{0\}$ be compact and let

$$
S \ni \xi \rightarrow K_{\xi} \subset \Gamma\left(T_{\xi} P h, \vartheta\right)
$$

be an outer continuous function whose values are compact sets. Then

$$
\varrho>0, \zeta \in \mathbf{R}^{n}, \pi \in K_{\xi}, \operatorname{Im} t \neq 0 \Rightarrow h(\varrho(\xi+\zeta+t \eta)) \neq 0
$$

for all $\xi \in S$ and all sufficiently small $\zeta, t, \varrho$.

Proof. Since the function $\xi \rightarrow \Gamma\left(T_{\xi} P h, \vartheta\right)$ is inner continuous and $S$ is compact, there is an outer continuous function

$$
S \ni \xi \rightarrow K_{\xi}^{*} \subset \Gamma\left(T_{\xi} \mathrm{Ph}, \vartheta\right)
$$

such that $K_{\xi}^{*}$ is a neighbourhood of $K_{\xi}$ for all $\xi$. We could for instance let $K_{\xi}^{*}$ consist of all $\eta$ whose distance to $K_{\xi}$ is $\leqslant \varepsilon$ where $\varepsilon>0$ is sufficiently small. Consider the function

$$
f(s, t, u, v)=\varrho^{-m} h(\varrho(\xi+\zeta+s \vartheta+t \eta))
$$

where $m$ is the degree of $h$ at the origin, $u=(\varrho, \zeta), \varrho \geqslant 0, \zeta \in \mathbf{R}^{n}, v=\eta \in K_{\xi}^{*}$ and $\xi \in S$ fixed. The hypotheses of the continuity lemma are then satisfied. In fact, the degree at 0 of

$$
t \rightarrow f(0, t, 0, v)=P h(\xi+t \eta)
$$

does not depend on $\eta$ and, according to the Main Lemma,

$$
\operatorname{Im} t \operatorname{Im} s \geqslant 0, \operatorname{Im} s>0 \Rightarrow f(s, t, 0, v)=P h(\xi+s \vartheta+t \eta) \neq 0
$$

and, finally,

$\operatorname{Im} s \neq 0, \operatorname{Im} t=0 \Rightarrow f(s, t, u, v) \neq 0$ 
when $\varrho, s, t, \zeta$ are small enough. Hence, by the continuity lemma, (15.18) holds when $\xi$ is fixed, $\eta \in K_{\xi}^{*}$ and $\zeta, \varrho, t$ are sufficiently small depending on $\xi$. But $K_{\xi}^{*} \supset K_{\xi+\zeta}$ when $\zeta \ni \mathbf{R}^{n}$ is sufficiently small and hence

$$
\varrho>0, \zeta \in \mathbf{R}^{n}, \eta \in K_{\xi+\zeta}, \operatorname{Im} t \neq 0 \Rightarrow h(\varrho(\xi+\zeta+t \eta)) \neq 0
$$

for every $\xi \in S$ when $\zeta, t, \varrho$ are sufficiently small depending on $\xi$. A covering of $S$ completes the proof.

Finally we shall give the lemma in a form convenient in the applications.

THEOREM (15.19). Let $h \in \mathrm{Hyp}_{\mathrm{loc}}(\vartheta)$, let $S$ be a compact part of $\mathbf{R}^{n}-\{0\}$ and let

$$
S \ni \xi \rightarrow K_{\xi} \subset \Gamma\left(T_{\xi} P h, \vartheta\right)
$$

be as in the previous lemma. Then

$$
\varrho>0, \xi \in S, \eta \in K_{\xi}, \zeta \in \mathbf{C}^{n}, \operatorname{Im} t \neq 0 \Rightarrow h(\varrho(\xi+\zeta+t \eta)) \neq 0
$$

when $\varrho, t, \operatorname{Re} \zeta$ and $\operatorname{Im} \zeta / \operatorname{Im} t$ are small enough.

Proof. We have

$$
\xi+\zeta+t \eta=\xi+\operatorname{Re}(\zeta+t \eta)+i \operatorname{Im} t\left(\eta+\operatorname{Im} \zeta(\operatorname{Im} t)^{-1}\right)
$$

so that the theorem follows immediately from the lemma.

\section{References}

[1]. Andersson, K. G., Propagation of analyticity of solutions of partial differential equation with constant coefficients. Ark. Mat., 8 (27) (1970), 277-302.

[2]. Atiyah, M. F., BotT, R. \& GÅrding, L., Lacunas for hyperbolic differential operators with constant coefficients I. Acta Math., 124 (1970), 109-189.

[3]. Aтiyah, M. F. \& Hodae, W. D. V., Integrals of the second kind of an algebraic variety. Ann. of Math., 62 (1955), 56-91.

[4]. Bazer, J. \& YeN, D. H. Y., The Riemann matrix of $(2+1)$-dimensional symmetrichyperbolic systems. Comm. Pure Appl. Math., 20 (1967), 329-363.

[5]. Bony, J. M. \& Schaprra, P., Existence et prolongement des solutions analytiques des systèmes hyperboliques non stricts. C. R. Acad. Sci. Paris Sér. A.-B., 274 (1972), 86-89.

[6]. Вотт, R., Homogeneous vector bundles. Ann. of Math., 66 (1957), 203-255.

[7]. Cartan, H. \& Eilengerg, S., Homological algebra. Princeton 1956.

[8]. Gårding, L., Local hyperbolicity. Israel J. Math., 13 (1972), 65-81.

[9]. Godement, R., Topologie Algebrique et Theorie des Faisceaux. Paris 1958.

[10]. Griffiths, P. H. A., On the periods of certain rational integrals I, II. Ann. of Math., 90 (1969), 460-541.

[11]. Grothendieck, A., Eléments de géometrie algébrique III. Étude cohomologique des faisceaux cohérents. Publ. IHES, 17 (1963), 5-91. 
[12]. - On the de Rham cohomology of algebraic varieties. Publ. IHEऽ, 29 (1966), 351-359.

[13]. Hartshorne, R., Ample vector bundles. Publ. IHES, 29 (1966), 63-94.

[14]. Hironaka, H., Resolution of singularities of an algebraic variety over a field of characteristic zero I, II. Ann. of Math., 79 (1964), 109-326.

[15]. Mathisson, M., Le problème de Hadamard relatif à la diffusion des ondes. Acta Math., 71 (1939), 249-282.

[16]. Petrovsky, I. G., On the diffusion of waves and the lacunas for hyperbolic equations. Mat. Sb., 17 (59) (1945). 289-370.

[17]. Serre, J.-P., Faisceux algébriques cohérents. Ann. of Math. 61 (1955), 197-278.

[18]. - Un théorème de dualité. Comment. Math. Helv., 29 (1955), 9-26.

[19]. Géometrie algébrique et géometrie analytique. Ann. Inst. Fourier, 6 (1956), 1-42.

[20]. Svensson, S. L., Necessary and sufficient conditions for the hyperbolicity of polynomials with hyperbolic principal part. Ark. Mat., 8 (17) (1969), 145-162.

Received March 27, 1973. 